\title{
W $^{\mathbf{V}}$ e phenomena

\section{A uniformly exponentially stable ADI scheme for Maxwell equations}

Konstantin Zerulla

CRC Preprint 2019/15, September 2019

\section{KARLSRUHE INSTITUTE OF TECHNOLOGY}

\section{CRC 1173}

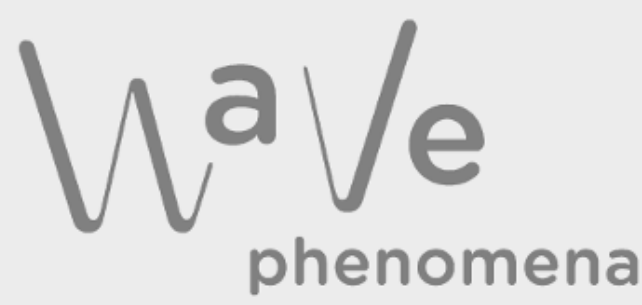




\section{Participating universities}

EBERHARD KARLS UNIVERSITATT TUBINGEN
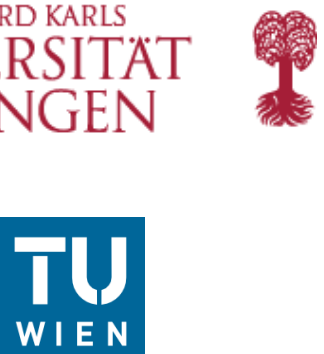

Funded by

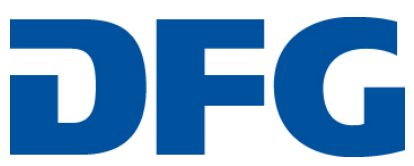




\title{
A UNIFORMLY EXPONENTIALLY STABLE ADI SCHEME FOR MAXWELL EQUATIONS
}

\author{
KONSTANTIN ZERULLA
}

\begin{abstract}
A modified alternating direction implicit scheme for the time integration of linear isotropic Maxwell equations with strictly positive conductivity on cuboids is constructed. A key feature of the proposed scheme is its uniform exponential stability, being achieved by coupling the Maxwell system with an additional damped PDE and adding artificial damping to the scheme. The implicit steps in the resulting time integrator further decouple into essentially one-dimensional elliptic problems, requiring only linear complexity. The convergence of the scheme to the solution of the original Maxwell system is analyzed in the abstract time-discrete setting, providing an error bound in a space related to $H^{-1}$.
\end{abstract}

\section{INTRODUCTION}

The Maxwell equations belong to the fundamental formulas of physics as they give account of the propagation of electromagnetic waves in matter. In view of many applications like wave guides, the efficient and reliable numerical solution of Maxwell equations is important.

For the time integration of linear isotropic Maxwell systems on domains with tensor structure, alternating direction implicit (ADI) schemes are very attractive. Particular instances are the Peaceman-Rachford ADI scheme and an ADI scheme that is energy conserving if the homogeneous Maxwell system is considered without damping, see $[33,5]$. If coupled with a finite difference grid or a discontinuous Galerkin discretization in space, these methods have the advantage that the degrees of freedom can be arranged in such a way that essentially only tridiagonal systems have to be solved in each step, see $[23,19]$. This eventually results in linear complexity. Another valuable property of both ADI schemes is their numerical stability. The implicit parts in both methods lead to unconditional stability without restriction on the time step size. A recent rigorous error analysis for the mentioned ADI schemes in the time-discrete setting is contained in $[18,9,10,8]$.

Despite their favorable efficiency and stability properties, it is however not clear whether ADI schemes preserve the decay properties of a damped Maxwell system, see the investigations in $[14,30]$ for other wave equations. The goal of this paper is thus to construct a modified ADI scheme which conserves the long-time behavior of a continuous Maxwell problem with damping.

Throughout, the focus lies on the linear isotropic Maxwell system with Ohm's law

$$
\begin{aligned}
\partial_{t} \mathbf{E} & =\frac{1}{\varepsilon} \operatorname{curl} \mathbf{H}-\sigma \mathbf{E} & & \text { in } Q \times[0, \infty), \\
\partial_{t} \mathbf{H} & =-\frac{1}{\mu} \operatorname{curl} \mathbf{E} & & \text { in } Q \times[0, \infty), \\
\operatorname{div}(\mu \mathbf{H}) & =0 & & \text { in } Q \times[0, \infty), \\
\mathbf{E} \times \nu & =0, \quad \mu \mathbf{H} \cdot \nu=0 & & \text { on } \partial Q \times[0, \infty), \\
\mathbf{E}(0) & =\mathbf{E}_{0}, \quad \mathbf{H}(0)=\mathbf{H}_{0} & & \text { in } Q,
\end{aligned}
$$

on a cuboid $Q=\left(a_{1}^{-}, a_{1}^{+}\right) \times\left(a_{2}^{-}, a_{2}^{+}\right) \times\left(a_{3}^{-}, a_{3}^{+}\right)$with perfectly conducting boundary $\partial Q$. The arising physical quantities are the electric field $\mathbf{E}(x, t) \in \mathbb{R}^{3}$, the magnetic field $\mathbf{H}(x, t) \in \mathbb{R}^{3}$, the conductivity $\sigma(x)>0$, the electric

Date: September 10, 2019.

2010 Mathematics Subject Classification. 35Q61, 93D99, 93B07, 47D06, 65M15.

Key words and phrases. Maxwell equations, splitting method, exponential stability, observability, error bound, divergence cleaning. Funded by the Deutsche Forschungsgemeinschaft (DFG, German Research Foundation) - Project-ID 258734477 - SFB 1173. 
permittivity $\varepsilon(x)>0$, and the magnetic permeability $\mu(x)>0$. The vector $\nu \in \mathbb{R}^{3}$ throughout denotes the unit outer normal vector at $\partial Q$.

For the coefficients we assume

$$
\varepsilon, \sigma \in \mathrm{W}^{1, \infty}(Q), \quad \mu \in \mathrm{W}^{1, \infty}(Q) \cap \mathrm{W}^{2,3}(Q) \quad \text { with } \varepsilon, \mu, \sigma \geq \delta>0 \quad \text { and } \frac{\partial \mu}{\partial \nu}=0 \text { on } \partial Q,
$$

employing a positive constant $\delta$. The slightly stronger regularity and the homogeneous boundary conditions for $\mu$ are needed because of technical reasons, see for instance the proof of Lemma 3.13. The initial data $\left(\mathbf{E}_{0}, \mathbf{H}_{0}\right)$ is required to be contained in $\mathrm{H}_{0}(\operatorname{curl}, Q) \times \mathrm{H}(\operatorname{curl}, Q)$ with

$$
\operatorname{div}\left(\varepsilon \mathbf{E}_{0}\right) \in \mathrm{L}^{2}(Q), \quad \operatorname{div}\left(\mu \mathbf{H}_{0}\right)=0 \text { on } Q, \quad \mu \mathbf{H}_{0} \cdot \nu=0 \text { on } \partial Q,
$$

where the involved spaces are recalled in Section 2.

In this situation, system (1.1) is known to have a unique classical solution $(\mathbf{E}, \mathbf{H})$ if interpreted as an evolution equation, see e.g. Proposition 2.3 of [9]. Regarding the long-time behavior of the solution, it is well known that the energy

$$
\mathscr{E}(t)=\frac{1}{2} \int_{Q} \varepsilon|\mathbf{E}(x, t)|^{2}+\mu|\mathbf{H}(x, t)|^{2} \mathrm{~d} x, \quad t \geq 0
$$

obeys the relation

$$
\mathscr{E}(t) \leq C \mathrm{e}^{-\beta t} \mathscr{E}(0), \quad t \geq 0
$$

for positive constants $C, \beta$ being independent of the initial data. This means that (1.1) is exponentially stable. For the stability result, we refer for instance to [27] in the case of smooth domains, and to [26] in the case of $\mathrm{C}^{2}$-domains. Recently, the decay property (1.3) was established in [11] for Lipschitz domains and more general uniformly positive definite matrix-valued coefficients $\varepsilon, \mu, \sigma \in \mathrm{L}^{\infty}$.

The decay property (1.3) of system (1.1) is caused by the damping term $-\sigma \mathbf{E}$ corresponding to Ohm's law. The latter solely affects the evolution of the electric field in a direct way, but then the coupling in (1.1) transports this effect also to the divergence-free parts of the magnetic field. It is thus essential for the exponential decay (1.3) that the magnetic field contains no curl-free parts that would otherwise be conserved over time.

Since relation (1.3) is a significant attribute of the Maxwell system (1.1), a reasonable time integrator for simulations over large time intervals should preserve the uniform exponential decay of the energy. The requirement of uniformity means here that the energy's decay rate is to be independent both of the initial data and the chosen time step size. It is however unlikely that approximations to (1.1) obtained by the ADI schemes from [33] and [5] share the desired uniform exponential decay property. This is due to divergence errors in the numerical approximation of the magnetic field that cannot be controlled on large time intervals, see for instance [5] for an investigation of divergence errors in numerical simulations. In view of the reasoning above, the damping effected by Ohm's law is consequently not strong enough to damp the entire electromagnetic field and cause a uniform exponential decay of the energy of the numerical approximation.

Similar phenomena have already been discussed in the literature for other wave equations and abstract evolution equations. Here a widely used remedy is artificial viscous damping that is included in the space or time discretization to damp out high frequency oscillations in the numerical approximation. The latter artifacts cause a loss of uniform exponential stability in the discrete system, meaning that the decay rate now depends on the space or time discretization, see for example [1, 14, 28, 30]. We also refer to [34] for a survey on the strongly related concept of observability for discretized wave equations. The paper [25] deals with the space discretization of a linear Maxwell system on a cube. Nicaise shows here that an observability inequality for the undamped continuous system does not uniformly hold for the space discretization.

Although the technique of artificial damping is widely successfully applied to preserve uniform exponential decay properties, there so far seems to be no efficiency and rigorous error analysis yielding precise convergence rates for the modified schemes. Convergence results without error estimates are for instance derived in [1,30].

In the spirit of the above mentioned papers, we use the technique of artificial viscous damping to construct a modification of an ADI scheme from [5]. The usual damping approaches, however, are not sufficient for the divergence artefacts in the magnetic field approximations. Therefore, we additionally apply a mixed hyperbolic 
divergence cleaning technique, proposed for MHD equations by Dedner et al. in [7] to account for numerical artefacts affecting the divergence constraint for $\mathbf{H}$. We modify this approach to damp the spurious curl-free parts of the magnetic field approximations already on the PDE level. To that end, we introduce an extended Maxwell system by coupling the original differential equation for the magnetic field with a new damped differential equation for an artificial variable.

The proposed uniformly exponentially stable ADI scheme is then formulated as a perturbed time integrator for the enlarged Maxwell system, see (3.18). To keep the favorable linear complexity of usual ADI schemes, we adapt the technique of artificial damping to splitting methods. More precisely, we employ for every substep in the splitting procedure damping operators only involving the current splitting operator. In this way, the application of the damping requires only linear computational effort, see Remark 3.17.

The exponential stability result is then stated in Theorem 3.9. The guiding principle for the corresponding proof is to establish an observability estimate for a similar energy conserving scheme, and to compare both methods in a final step to derive the desired energy decay. This technique has also been successfully applied in [30] for the oneand two-dimensional wave equation. The main task thereby consists in the derivation of the uniform observability inequality. Regarding continuous systems, microlocal analysis and the multiplier method are useful to deduce observability, see for instance $[3,11,27,22,32,26]$. Observability of discrete systems is often proved via a discrete multiplier method or a spectral filtering technique, compare $[25,13,14,28,1]$. Below, we proceed with the first technique since it fits best to our discrete scheme, and we transfer arguments from [26] to the time-discrete setting.

Although the proposed scheme approximates the enlarged Maxwell system, it in fact converges against the solution of the original system (1.1), provided that appropriate initial data are chosen, see Theorem 6.5. This convergence result is derived via a wellposedness argument for the extended Maxwell system and a rigorous error analysis using techniques from [8]. In this way we show that the proposed scheme converges with order one in $\mathrm{H}^{-1}$, roughly speaking. The order of convergence for the proposed scheme is here limited to 1 , because the Maxwell system is split into 6 subproblems, compared to the number of 2 subsystems in the standard splittings in $[8,18,9,10]$. The crucial property of the error statement is that it requires only regularity of the initial data but not of the unknown solution of the continuous problem. This is achieved by means of a regularity analysis for the enlarged Maxwell system.

Despite the abstract time-discrete setting, similar arguments are likely to yield the respective error result also for the full discretization by the method of lines, involving for instance a discontinuous Galerkin (dG) method for space discretization. Concerning the exponential decay result, one has to investigate whether a dG discretization with stabilizing upwind fluxes in space, and the proposed ADI scheme in time are sufficient to preserve the uniform exponential decay. Similar reasoning is for instance employed in [14], where a fully discrete numerical scheme with exponentially stable approximations is derived for a one-dimensional damped wave equation.

To fix a common framework, the next section recalls basic spaces related to the linear Maxwell equations as well as some useful notation. In Section 3 we introduce the extended Maxwell system, derive its wellposedness as a Cauchy problem, and present the new ADI scheme as well as the uniform exponential stability result. The succeeding Section 4 is devoted to the derivation of an observability estimate for an energy conserving scheme being similar to the desired damped one. The actual exponential stability result is then proved in Section 5 by means of the observability inequality. A rigorous error analysis is finally performed in Section 6 .

\section{AnAlytical PRELIMINARIES AND NOtATION}

This section has auxiliary purposes, as it recalls the standard function spaces for linear Maxwell equations and introduces our notation. The starting point are the maximal domains

$$
\begin{aligned}
\mathrm{H}(\operatorname{curl}, Q) & :=\left\{\varphi \in \mathrm{L}^{2}(Q)^{3} \mid \operatorname{curl} \varphi \in \mathrm{L}^{2}(Q)^{3}\right\}, \quad\|\varphi\|_{\operatorname{curl}}^{2}:=\|\varphi\|_{\mathrm{L}^{2}}^{2}+\|\operatorname{curl} \varphi\|_{\mathrm{L}^{2}}^{2}, \\
\mathrm{H}(\operatorname{div}, Q) & :=\left\{v \in \mathrm{L}^{2}(Q)^{3} \mid \operatorname{div} v \in \mathrm{L}^{2}(Q)\right\}, \quad\|v\|_{\text {div }}^{2}:=\|v\|_{\mathrm{L}^{2}}^{2}+\|\operatorname{div} v\|_{\mathrm{L}^{2}}^{2},
\end{aligned}
$$

which are complete for the respective norms. The subspaces $\mathrm{H}_{0}(\operatorname{curl}, Q)$ and $\mathrm{H}_{0}(\operatorname{div}, Q)$ further denote the completion of the space of test functions $\mathrm{C}_{c}^{\infty}(Q)^{3}$ on $Q$ with respect to the norms $\|\cdot\|_{\text {curl }}$ and $\|\cdot\|_{\text {div }}$, respectively. Concerning boundary traces in $\mathrm{H}(\operatorname{curl}, Q)$, Theorems 2.11 and 2.12 in Section I.2 of [15] yield that vectors in $\mathrm{H}(\operatorname{curl}, Q)$ have a well-defined tangential trace on the boundary of $Q$, and that the tangential trace operator is 
continuous from $\mathrm{H}(\operatorname{curl}, Q)$ into $\mathrm{H}^{-1 / 2}(\partial Q)$. The subspace $\mathrm{H}_{0}(\operatorname{curl}, Q)$ furthermore coincides with the vectors in $\mathrm{H}($ curl,$Q)$ having zero tangential trace on $\partial Q$. Similar results are provided by Theorems 2.5 and 2.6 in Section I.2 of [15] for the divergence. Functions in $\mathrm{H}(\operatorname{div}, Q)$ have a well-defined normal trace on the boundary $\partial Q$, and the normal trace operator is continuous from $\mathrm{H}(\operatorname{div}, Q)$ into $\mathrm{H}^{-1 / 2}(\partial Q)$ with kernel $\mathrm{H}_{0}(\operatorname{div}, Q)$.

The intersections

$$
\mathrm{H}_{T}(\operatorname{curl}, \operatorname{div}, Q):=\mathrm{H}(\operatorname{curl}, Q) \cap \mathrm{H}_{0}(\operatorname{div}, Q), \quad \mathrm{H}_{N}(\operatorname{curl}, \operatorname{div}, Q):=\mathrm{H}_{0}(\operatorname{curl}, Q) \cap \mathrm{H}(\operatorname{div}, Q),
$$

are valuable for decompositions of the electric and magnetic fields. Both spaces are complete with respect to the norm

$$
\|\varphi\|_{\mathrm{H}_{T}}^{2}:=\|\varphi\|_{\mathrm{H}_{N}}^{2}:=\|\operatorname{curl} \varphi\|_{\mathrm{L}^{2}}^{2}+\|\operatorname{div} \varphi\|_{\mathrm{L}^{2}}^{2},
$$

and embed continuously into $\mathrm{H}^{1}(Q)^{3}$, meaning that there is a uniform constant $C_{T}>0$ with

$$
\|\varphi\|_{\mathrm{H}^{1}}^{2} \leq C_{T}\left(\|\operatorname{curl} \varphi\|_{\mathrm{L}^{2}}^{2}+\|\operatorname{div} \varphi\|_{\mathrm{L}^{2}}^{2}\right)
$$

for all vectors $\varphi \in \mathrm{H}_{T}(\operatorname{curl}, \operatorname{div}, Q) \cup \mathrm{H}_{N}(\operatorname{curl}$, div, $Q)$, see Lemmas 3.4, 3.6, and Theorems 3.7, 3.9 in Section I.3 of $[15]$.

The proof of an observability estimate will heavily make use of appropriate Helmholtz decompositions. One is established in Theorem 3.6 and Corollary 3.4 in Section I.3 of [15], and it provides for each vector $g \in \mathrm{L}^{2}(Q)^{3}$ the orthogonal decomposition

$$
g=\operatorname{curl} \phi+\nabla q
$$

with a function $q \in \mathrm{H}^{1}(Q)$ unique up to a constant, and a vector $\phi \in \mathrm{H}^{1}(Q)^{3}$ satisfying $\operatorname{div} \phi=0$, curl $\phi \in \mathrm{H}_{0}(\operatorname{div}, Q)$ and $\phi \times \nu=0$ on $\partial Q$. In the following, $p_{\nabla}$ and $p_{\text {curl }}$ denote the orthogonal projections of a function in $\mathrm{L}^{2}(Q)^{3}$ onto its curl-free and divergence-free part, according to $(2.2)$.

For a normed vector space $\left(X,\|\cdot\|_{X}\right)$, the space of bounded linear operators on $X$ is denoted by $\mathscr{B}(X)$, and the corresponding operator norm by $\|\cdot\|_{\mathscr{B}(X)}$. Let $Y$ further be a subspace of $X$, and $A$ a linear operator on $X$ with domain $\mathcal{D}(A)$. The graph norm of $A$ is then defined via $\|x\|_{\mathcal{D}(A)}^{2}:=\|x\|_{X}^{2}+\|A x\|_{X}^{2}$ for $x \in \mathcal{D}(A)$, and the part of $A$ in $Y$ is given by $A_{Y} y:=A y$ for $y \in \mathcal{D}\left(A_{Y}\right):=\{y \in Y \mid y \in \mathcal{D}(A), A y \in Y\}$. If $X$ is additionally a Hilbert space and $A$ is densely defined, positive and selfadjoint, one can define fractional extrapolation spaces $\left(X_{\alpha}^{A}\right)_{\alpha \in \mathbb{R} \backslash\{0\}}$ of $X$ with respect to $A$. The part of $A$ in $X_{\alpha}^{A}$ is denoted by $A_{\alpha}$ for $\alpha \in \mathbb{R} \backslash\{0\}$, see Sections V.1.3 and V.1.4 in [2] and Section 2.10 in [31] for extrapolation spaces.

Finally, spaces of partial regularity and the need for well-defined traces on parts of the boundary $\partial Q$ naturally arise when dealing with ADI splitting schemes. The boundary is therefore subdivided into the parts

$$
\Gamma_{j}:=\left\{x \in \partial Q \mid x_{j} \in\left\{a_{j}^{-}, a_{j}^{+}\right\}\right\},
$$

and the corresponding trace operator $\operatorname{tr}_{\Gamma_{j}}=\left.\operatorname{tr}\right|_{\Gamma_{j}}$ is continuously extended from $\mathrm{H}^{1}(Q)$ to the space of partial regularity $\mathcal{D}\left(\partial_{j}\right):=\left\{f \in \mathrm{L}^{2}(Q) \mid \partial_{j} f \in \mathrm{L}^{2}(Q)\right\}$ for $j \in\{1,2,3\}$, see Section 2 in [9]. We will simply write $v=0$ on $\Gamma_{j}$ instead of $\operatorname{tr}_{\Gamma_{j}} v=0$ for a function $v \in \mathcal{D}\left(\partial_{j}\right)$.

Convention: Whenever a differential operator is applied to a product of two functions and parentheses are omitted, the product rule is tacitly applied. This means for instance $\partial_{x} f g=\partial_{x}(f g)=\left(\partial_{x} f\right) g+\left(\partial_{x} g\right) f$ for functions $f, g \in \mathrm{H}^{1}(\mathbb{R})$.

\section{Construction of a Uniformly Exponentially StABle ADI SCHEME}

To stabilize system (1.1) already on the PDE level, an extended Maxwell system is introduced in this section, and the connection to the original system (1.1) is made precise. The newly proposed ADI scheme is afterwards constructed as a perturbed time integrator for the extended system, and the uniform exponential stability result is stated in Theorem 3.9. This section is concluded with a detailed regularity analysis for the splitting operators which will become crucial for the error analysis and the proof of an observability estimate. 


\subsection{An extended Maxwell system}

We consider the coupled linear PDE system

$$
\begin{aligned}
\partial_{t} \mathbf{E} & =\frac{1}{\varepsilon} \operatorname{curl} \mathbf{H}-\sigma \mathbf{E} & & \text { in } Q \times[0, \infty), \\
\partial_{t} \mathbf{H} & =-\frac{1}{\mu} \operatorname{curl} \mathbf{E}-\nabla\left(\frac{1}{\mu} \Phi\right) & & \text { in } Q \times[0, \infty), \\
\partial_{t} \Phi & =-\frac{1}{\mu^{2}} \operatorname{div}(\mu \mathbf{H})-\eta \Phi & & \text { in } Q \times[0, \infty), \\
\mathbf{E} \times \nu & =0, \quad \mu \mathbf{H} \cdot \nu=0 & & \text { on } \partial Q \times[0, \infty), \\
\mathbf{E}(0) & =\mathbf{E}_{0}, \quad \mathbf{H}(0)=\mathbf{H}_{0}, \quad \Phi(0)=\Phi_{0} & & \text { in } Q,
\end{aligned}
$$

which we call extended or enlarged Maxwell system. The new artificial quantity $\Phi=\Phi(x, t) \in \mathbb{R}$ acts here as a counterpart to the electric field. The term $-\operatorname{curl} \mathbf{E}$ namely influences the divergence-free parts of $\mu \mathbf{H}$, whereas the gradient of $\frac{1}{\mu} \Phi$ affects the curl-free parts of $\mathbf{H}$, roughly speaking. When establishing the observability of an associated time-discrete system, the influence of the vector $\nabla\left(\frac{1}{\mu} \Phi\right)$ is essential for a control of the curl-free parts of $\mathbf{H}$, see Lemma 4.6 .

The initial datum $\Phi_{0}$ is assumed to belong to $\mathrm{H}^{1}(Q)$, and the damping coefficient $\eta=\eta(x)>0$ shall satisfy

$$
\eta \in \mathrm{W}^{1, \infty}(Q) \quad \text { with } \eta \geq \delta
$$

In view of the application of the gradient and divergence operators on $\mu$ in (3.1), we have to impose somewhat higher regularity assumptions in (1.2) on $\mu$ than on $\varepsilon$ and $\sigma$. During the analysis of the splitting operators corresponding to the gradient and divergence, we indeed need higher regularity requirements on $\mu$ to control the Hessian $\partial^{2} \mu$ in $\mathrm{L}^{3}(Q)$, see the proof of Lemma 3.13. Note further that the electric field and the function $\Phi$ receive a direct damping due to (1.2) and (3.2). Another crucial novelty about (3.1) with regards to (1.1) is the absence of Gauss' law $\operatorname{div}(\mu \mathbf{H})=0$. This represents the phenomenon that approximations to the magnetic field obtained by the considered ADI schemes are usually not divergence-free.

Despite its different appearance, system (3.1) reduces to the original Maxwell system (1.1) if the initial data are chosen physically reasonable, see Remark 3.6. This is significant for numerical approximations to (3.1) since usually only approximations to (1.1) are desirable.

System (3.1) is in the following interpreted as an evolution equation on the space $X=\mathrm{L}^{2}(Q)^{7}$, being a Hilbert space with respect to the weighted inner product

$$
\left(\left(\begin{array}{c}
\mathbf{E}^{1} \\
\mathbf{H}^{1} \\
\Phi^{1}
\end{array}\right),\left(\begin{array}{c}
\mathbf{E}^{2} \\
\mathbf{H}^{2} \\
\Phi^{2}
\end{array}\right)\right):=\int_{Q}\left(\varepsilon \mathbf{E}^{1} \cdot \mathbf{E}^{2}+\mu \mathbf{H}^{1} \cdot \mathbf{H}^{2}+\mu \Phi^{1} \Phi^{2}\right) \mathrm{d} x
$$

that induces the norm $\|\cdot\|$ on $X$. Due to the assumptions (1.2) and (3.2), this norm is equivalent to the $\mathrm{L}^{2}$-norm on $Q$. The norm $\|\cdot\|$ will often be called energy. The corresponding extended Maxwell operator is defined as

$$
M_{\text {ext }}\left(\begin{array}{c}
\mathbf{E} \\
\mathbf{H} \\
\Phi
\end{array}\right):=\left(\begin{array}{c}
\frac{1}{\varepsilon} \operatorname{curl} \mathbf{H}-\sigma \mathbf{E} \\
-\frac{1}{\mu} \operatorname{curl} \mathbf{E}-\nabla\left(\frac{1}{\mu} \Phi\right) \\
-\frac{1}{\mu^{2}} \operatorname{div}(\mu \mathbf{H})-\eta \Phi
\end{array}\right), \quad \mathcal{D}\left(M_{\text {ext }}\right):=\mathrm{H}_{0}(\operatorname{curl}, Q) \times \mathrm{H}_{T}(\operatorname{curl}, \operatorname{div}, Q) \times \mathrm{H}^{1}(Q)
$$

Concerning the coefficients $\varepsilon$ and $\mu$ in the regularity and boundary conditions, a remark is in order.

Remark 3.1. The function $\operatorname{div}(\mu \mathbf{H})$ belongs to $\mathrm{L}^{2}(Q)$ if and only if $\operatorname{div} \mathbf{H}$ does because of the product rule $\operatorname{div}(\mu \mathbf{H})=(\nabla \mu) \cdot \mathbf{H}+\mu \operatorname{div} \mathbf{H}$ and the assumptions (1.2) on $\mu$. A similar reasoning shows that the mapping $\frac{1}{\mu} \Phi$ is contained in $\mathrm{H}^{1}(Q)$ if and only if $\Phi$ is. Regarding the boundary condition for the magnetic field, consult Remark 3.3 in [18]. Analogous considerations also apply to the coefficient $\varepsilon$, and will frequently be used later on. 
Within this framework the extended system (3.1) is interpreted as the Cauchy problem

$$
\frac{\mathrm{d}}{\mathrm{d} t}\left(\begin{array}{c}
\mathbf{E} \\
\mathbf{H} \\
\Phi
\end{array}\right)=M_{\text {ext }}\left(\begin{array}{c}
\mathbf{E} \\
\mathbf{H} \\
\Phi
\end{array}\right), \quad t \geq 0, \quad\left(\begin{array}{c}
\mathbf{E}(0) \\
\mathbf{H}(0) \\
\Phi(0)
\end{array}\right)=\left(\begin{array}{c}
\mathbf{E}_{0} \\
\mathbf{H}_{0} \\
\Phi_{0}
\end{array}\right) \in \mathcal{D}\left(M_{\text {ext }}\right),
$$

posed on $X$. For the proof of the classical wellposedness of (3.3), the following density result is useful. Although it might be well known to experts, we give a proof to make the presentation self-contained.

Lemma 3.2. The space $\mathrm{H}_{T}(\operatorname{curl}, \operatorname{div}, Q)$ is dense in $\mathrm{H}(\operatorname{curl}, Q)$.

Proof. It suffices to show that every function $\varphi \in \mathrm{C}^{\infty}(\bar{Q})^{3}$ can be approximated by a sequence $\left(\varphi_{n}\right)_{n}$ in $\mathrm{C}^{\infty}(\bar{Q})^{3}$ satisfying $\varphi_{n} \cdot \nu=0$ on $\partial Q$, since the space $\mathrm{C}^{\infty}(\bar{Q})^{3}$ is dense in $\mathrm{H}(\operatorname{curl}, Q)$, see Theorem 2.10 in Section I.2 of [15].

Let $i \in\{1,2,3\}$ and $n \in \mathbb{N}$. We employ smooth cut-off functions $\chi_{n}^{i}:\left[a_{i}^{-}, a_{i}^{+}\right] \rightarrow[0,1]$ with compact support in $\left[a_{i}^{-}+\frac{1}{2 n}, a_{i}^{+}-\frac{1}{2 n}\right]$, that are equal to 1 on $\left[a_{i}^{-}+\frac{1}{n}, a_{i}^{+}-\frac{1}{n}\right]$. Denoting the $i$-th component of $\varphi$ by $\varphi^{i}$, we define the function

$$
\varphi_{n}(x):=\left(\chi_{n}^{i}\left(x_{i}\right) \varphi^{i}(x)\right)_{i=1}^{3}, \quad x=\left(x_{1}, x_{2}, x_{3}\right) \in \bar{Q} .
$$

Clearly, $\varphi_{n}$ is again smooth and satisfies $\varphi_{n} \cdot \nu=0$ on $\partial Q$. Lebesgue's dominated convergence theorem implies the convergence of $\left(\varphi_{n}\right)_{n}$ to $\varphi$ and of the functions

$$
\operatorname{curl} \varphi_{n}=\left(\begin{array}{c}
\chi_{n}^{3} \partial_{2} \varphi^{3}-\chi_{n}^{2} \partial_{3} \varphi^{2} \\
\chi_{n}^{1} \partial_{3} \varphi^{1}-\chi_{n}^{3} \partial_{1} \varphi^{3} \\
\chi_{n}^{2} \partial_{1} \varphi^{2}-\chi_{n}^{1} \partial_{2} \varphi^{1}
\end{array}\right)
$$

to $\operatorname{curl} \varphi$ in $\mathrm{L}^{2}(Q)^{3}$ as $n \rightarrow \infty$.

The next proposition yields the classical wellposedness of (3.3) in $X$, and its proof employs arguments from Proposition 3.1 in [18]. Also the undamped case $\sigma=\eta=0$ is considered, where the energy of the corresponding solutions is shown to be conserved. This is in agreement with the energy conservation of $(1.1)$ for $\sigma=0$, see Proposition 3.5 in [18].

Proposition 3.3. Let $\varepsilon, \mu$ satisfy (1.2). The following statements are true.

a) If $\sigma=\eta=0$, then $M_{\text {ext }}$ is skewadjoint, and generates a strongly continuous group of isometries on $X$.

b) Let $\sigma, \eta \geq 0$ be contained in $\mathrm{W}^{1, \infty}(Q)$. In this case $M_{\mathrm{ext}}$ is the generator of a contractive strongly continuous semigroup on $X$.

In both cases (3.3) has a unique classical solution $(\boldsymbol{E}, \boldsymbol{H}, \Phi) \in \mathrm{C}\left([0, \infty), \mathcal{D}\left(M_{\mathrm{ext}}\right)\right) \cap \mathrm{C}^{1}([0, \infty), X)$.

Proof. We show only the skewadjointness of $M_{\text {ext }}$ in the case $\sigma=\eta=0$. Stone's Theorem then yields the remainder of part a). The subsequent item is deduced via perturbation theory for generators of semigroups, see Theorem III.2.7 in [12]. The addendum is standard semigroup theory. So, let $\sigma=\eta=0$.

1) We split the extended Maxwell operator into

$$
M_{\text {ext }}\left(\begin{array}{c}
\mathbf{E} \\
\mathbf{H} \\
\Phi
\end{array}\right)=\left(\begin{array}{c}
\frac{1}{\varepsilon} \operatorname{curl} \mathbf{H} \\
-\frac{1}{\mu} \operatorname{curl} \mathbf{E}-\frac{1}{\mu} \nabla \Phi \\
-\frac{1}{\mu} \operatorname{div} \mathbf{H}
\end{array}\right)+\left(\begin{array}{c}
0 \\
\frac{1}{\mu^{2}}(\nabla \mu) \Phi \\
-\frac{1}{\mu^{2}}(\nabla \mu) \cdot \mathbf{H}
\end{array}\right)=: \tilde{M}_{\mathrm{ext}}\left(\begin{array}{c}
\mathbf{E} \\
\mathbf{H} \\
\Phi
\end{array}\right)+T\left(\begin{array}{c}
\mathbf{E} \\
\mathbf{H} \\
\Phi
\end{array}\right)
$$

for $(\mathbf{E}, \mathbf{H}, \Phi) \in \mathcal{D}\left(\tilde{M}_{\text {ext }}\right):=\mathcal{D}\left(M_{\text {ext }}\right)$, and set $\mathcal{D}(T):=X$. The assumptions (1.2) imply that $T$ is bounded and skewadjoint. Employing perturbation theory for selfadjoint operators, it suffices to show that $\tilde{M}_{\text {ext }}$ is skewadjoint, see Theorem V.4.3 in [21].

2) The operator $\tilde{M}_{\text {ext }}$ is clearly densely defined in $X$. The closedness of $\tilde{M}_{\text {ext }}$ can be seen by the following reasoning. Let $\left(\left(\mathbf{E}^{n}, \mathbf{H}^{n}, \Phi^{n}\right)\right)_{n}$ in $\mathcal{D}\left(\tilde{M}_{\text {ext }}\right)$, and $(\mathbf{E}, \mathbf{H}, \Phi),(\tilde{\mathbf{E}}, \tilde{\mathbf{H}}, \tilde{\Phi})$ in $X$ with $\left(\mathbf{E}^{n}, \mathbf{H}^{n}, \Phi^{n}\right) \rightarrow(\mathbf{E}, \mathbf{H}, \Phi)$ and $\tilde{M}_{\text {ext }}\left(\mathbf{E}^{n}, \mathbf{H}^{n}, \Phi^{n}\right) \rightarrow(\tilde{\mathbf{E}}, \tilde{\mathbf{H}}, \tilde{\Phi})$ in $X$ as $n \rightarrow \infty$. Recall the closedness of the operators div and curl on their maximal domains in $\mathrm{L}^{2}$, and the continuity of the normal trace operator from $\mathrm{H}(\operatorname{div}, Q)$ to $\mathrm{H}^{-1 / 2}(\partial Q)$. Employing these facts as well as the convergence of $\left(\operatorname{curl} \mathbf{H}^{n}\right)_{n}$ to $\varepsilon \tilde{\mathbf{E}}$ and of $\left(-\operatorname{div} \mathbf{H}^{n}\right)_{n}$ to $\mu \tilde{\Phi}$, the functions curl $\mathbf{H}$ and $\operatorname{div} \mathbf{H}$ belong to $\mathrm{L}^{2}, \mathbf{H} \cdot \nu=0$ on $\partial Q, \frac{1}{\varepsilon} \operatorname{curl} \mathbf{H}=\tilde{\mathbf{E}}$ and $-\frac{1}{\mu} \operatorname{div} \mathbf{H}=\tilde{\Phi}$. It remains to consider the second component of 
$\tilde{M}_{\text {ext }}\left(\mathbf{E}^{n}, \mathbf{H}^{n}, \Phi^{n}\right)$. Since (- $\left.\operatorname{curl} \mathbf{E}^{n}-\nabla \Phi^{n}\right)_{n}$ converges in the $\mathrm{L}^{2}$-sense, the continuity of the Helmholtz-projections implies that both sequences $\left(\operatorname{curl} \mathbf{E}^{n}\right)_{n}$ and $\left(\nabla \Phi^{n}\right)_{n}$ converge in $\mathrm{L}^{2}(Q)^{3}$. The closedness of the operators $\nabla$ and curl on their maximal domains, and the continuity of the tangential trace in $\mathrm{H}(\operatorname{curl}, Q)$ finally show that $\mathbf{E}$ belongs to $\mathrm{H}_{0}(\operatorname{curl}, Q), \Phi$ is contained in $\mathrm{H}^{1}(Q)$ and $-\frac{1}{\mu} \operatorname{curl} \mathbf{E}-\frac{1}{\mu} \nabla \Phi=\tilde{\mathbf{H}}$. As a result, $\tilde{M}_{\text {ext }}$ is closed on $X$.

3) Let $\left(\mathbf{E}^{1}, \mathbf{H}^{1}, \Phi^{1}\right),\left(\mathbf{E}^{2}, \mathbf{H}^{2}, \Phi^{2}\right) \in \mathcal{D}\left(\tilde{M}_{\text {ext }}\right)$. In view of the boundary conditions for the fields $\mathbf{E}^{1}, \mathbf{E}^{2}, \mathbf{H}^{1}, \mathbf{H}^{2}$, an integration by parts shows the identities

$$
\begin{aligned}
\left(\tilde{M}_{\text {ext }}\left(\begin{array}{c}
\mathbf{E}^{1} \\
\mathbf{H}^{1} \\
\Phi^{1}
\end{array}\right),\left(\begin{array}{c}
\mathbf{E}^{2} \\
\mathbf{H}^{2} \\
\Phi^{2}
\end{array}\right)\right) & =\int_{Q}\left(\left(\operatorname{curl} \mathbf{H}^{1}\right) \cdot \mathbf{E}^{2}-\left(\operatorname{curl} \mathbf{E}^{1}\right) \cdot \mathbf{H}^{2}-\left(\nabla \Phi^{1}\right) \cdot \mathbf{H}^{2}-\left(\operatorname{div} \mathbf{H}^{1}\right) \Phi^{2}\right) \mathrm{d} x \\
& =\int_{Q}\left(\mathbf{H}^{1} \cdot \operatorname{curl} \mathbf{E}^{2}-\mathbf{E}^{1} \cdot \operatorname{curl} \mathbf{H}^{2}+\Phi^{1} \operatorname{div} \mathbf{H}^{2}+\mathbf{H}^{1} \cdot \nabla \Phi^{2}\right) \mathrm{d} x \\
& =-\left(\left(\begin{array}{c}
\mathbf{E}^{1} \\
\mathbf{H}^{1} \\
\Phi^{1}
\end{array}\right), \tilde{M}_{\mathrm{ext}}\left(\begin{array}{c}
\mathbf{E}^{2} \\
\mathbf{H}^{2} \\
\Phi^{2}
\end{array}\right)\right) .
\end{aligned}
$$

As a result, $\tilde{M}_{\text {ext }}$ is skewsymmetric.

4) It now suffices to show that $I \pm \tilde{M}_{\text {ext }}$ has dense range to conclude the skewadjointness of $\tilde{M}_{\text {ext }}$. Let $(\check{\mathbf{E}}, \check{\mathbf{H}}, \check{\Phi}) \in$ $\mathrm{C}_{c}^{\infty}(Q)^{7}$, and consider the system

$$
\begin{aligned}
\mathbf{E} \pm \frac{1}{\varepsilon} \operatorname{curl} \mathbf{H} & =\check{\mathbf{E}}, \\
\mathbf{H} \mp \frac{1}{\mu} \operatorname{curl} \mathbf{E} \mp \frac{1}{\mu} \nabla \Phi & =\check{\mathbf{H}}, \\
\Phi \mp \frac{1}{\mu} \operatorname{div} \mathbf{H} & =\check{\Phi},
\end{aligned}
$$

corresponding to $\left(I \pm \tilde{M}_{\text {ext }}\right)(\mathbf{E}, \mathbf{H}, \Phi)=(\check{\mathbf{E}}, \check{\mathbf{H}}, \check{\Phi})$. Inserting the first and third line into the second, the formula

$$
\mu \mathbf{H}+\operatorname{curl} \frac{1}{\varepsilon} \operatorname{curl} \mathbf{H}-\nabla \frac{1}{\mu} \operatorname{div} \mathbf{H}=\mu \check{\mathbf{H}} \pm \operatorname{curl} \check{\mathbf{E}} \pm \nabla \check{\Phi}=: h \in \mathrm{W}^{1, \infty}(Q)^{3} \cap \mathrm{C}_{\mathrm{c}}(\mathrm{Q})^{3}
$$

follows. To find a solution of (3.7), it is useful to consider the weak formulation

$$
\int_{Q} \mu \mathbf{H} \cdot v+\frac{1}{\varepsilon}(\operatorname{curl} \mathbf{H}) \cdot(\operatorname{curl} v)+\frac{1}{\mu}(\operatorname{div} \mathbf{H})(\operatorname{div} v) \mathrm{d} x=\int_{Q} h v \mathrm{~d} x
$$

for $v \in \mathrm{H}_{T}(\operatorname{curl}$, div, $Q)$. The Lax-Milgram Lemma then provides a unique function $\mathbf{H} \in \mathrm{H}_{T}(\operatorname{curl}$, div, $Q)$ satisfying (3.8).

4.i) We first want to show that the function $\frac{1}{\mu} \operatorname{div} \mathbf{H}$ belongs to $\mathrm{H}^{2}(Q)$, and we therefore modify the proof of Theorem 1.1 in [6]. Let $\zeta$ be a function in $\mathrm{H}^{2}(Q)$ with $\frac{\mathrm{d} \zeta}{\mathrm{d} \nu}=0$ on $\partial Q$. Testing (3.8) with $v:=\nabla \zeta$, we infer the equation

$$
\int_{Q} \frac{1}{\mu}(\operatorname{div} \mathbf{H})(\Delta \zeta) \mathrm{d} x=-\int_{Q}(\operatorname{div} h-\operatorname{div}(\mu \mathbf{H})) \zeta \mathrm{d} x
$$

By Theorem 3.2.1.3 in [16], the Neumann problem

$$
\begin{aligned}
-\Delta \hat{u}+\hat{u} & =\operatorname{div} h-\operatorname{div}(\mu \mathbf{H})+\frac{1}{\mu} \operatorname{div} \mathbf{H} & & \text { in } Q, \\
\frac{\mathrm{d} \hat{u}}{\mathrm{~d} \nu} & =0 & & \text { on } \partial Q,
\end{aligned}
$$

has a unique solution $\hat{u} \in \mathrm{H}^{2}(Q)$, satisfying the formula

$$
\int_{Q} \hat{u} \Delta \zeta-\hat{u} \zeta \mathrm{d} x=-\int_{Q}\left(\operatorname{div} h-\operatorname{div}(\mu \mathbf{H})+\frac{1}{\mu} \operatorname{div} \mathbf{H}\right) \zeta \mathrm{d} x .
$$


In view of (3.9), we consequently have derived the expression

$$
\left\langle\hat{u}-\frac{1}{\mu} \operatorname{div} \mathbf{H}, \Delta \zeta-\zeta\right\rangle_{\mathrm{L}^{2}}=0 .
$$

Since the range of the operator $\Delta-I$ with domain $\left\{\Phi \in \mathrm{H}^{2}(Q) \mid \frac{\mathrm{d} \Phi}{\mathrm{d} \nu}=0\right.$ on $\left.\partial Q\right\}$ coincides with $\mathrm{L}^{2}(Q)$ by Theorem 3.2.1.3 in [16], we infer $\frac{1}{\mu} \operatorname{div} \mathbf{H}=\hat{u} \in \mathrm{H}^{2}(Q)$ and $\frac{\mathrm{d}}{\mathrm{d} \nu}\left(\frac{1}{\mu} \operatorname{div} \mathbf{H}\right)=0$ on $\partial Q$.

4.ii) It remains to deduce that the vector $\frac{1}{\varepsilon} \operatorname{curl} \mathbf{H}$ belongs to $\mathrm{H}_{0}(\operatorname{curl}, Q)$. Applying an integration by parts to (3.8), the integral equation

$$
\int_{Q} \frac{1}{\varepsilon}(\operatorname{curl} \mathbf{H}) \cdot(\operatorname{curl} v) \mathrm{d} x=\int_{Q}\left(h-\mu \mathbf{H}+\nabla\left(\frac{1}{\mu} \operatorname{div} \mathbf{H}\right)\right) \cdot v \mathrm{~d} x
$$

follows for all vectors $v \in \mathrm{H}_{T}(\operatorname{curl}, \operatorname{div}, Q)$. This means that $\frac{1}{\varepsilon} \operatorname{curl} \mathbf{H}$ is an element of $\mathrm{H}(\operatorname{curl}, Q)$. Lemma 3.2 further yields that the relation is true even for all $v \in \mathrm{H}(\operatorname{curl} Q)$, and Lemma 2.4 in Section I of [15] implies that $\frac{1}{\varepsilon} \operatorname{curl} \mathbf{H}$ is contained in $\mathrm{H}_{0}(\operatorname{curl}, Q)$. Thus, $\mathbf{H}$ solves $(3.7)$ in $\mathrm{L}^{2}(Q)^{3}$ in strong form.

4.iii) Let finally $\mathbf{E}:=\check{\mathbf{E}} \mp \frac{1}{\varepsilon} \operatorname{curl} \mathbf{H} \in \mathrm{H}_{0}(\operatorname{curl}, Q)$ and $\Phi:=\check{\Phi} \pm \frac{1}{\mu} \operatorname{div} \mathbf{H} \in \mathrm{H}^{1}(Q)$. The vector $(\mathbf{E}, \mathbf{H}, \Phi)$ then belongs to $\mathcal{D}\left(\tilde{M}_{\text {ext }}\right)$ and solves $(3.4)-(3.6)$.

In general, the solutions of (3.1) do not solve the original system (1.1). For appropriate initial data, however, the associated solutions indeed satisfy the original problem. In the following this is deduced by means of subspace theory for semigroups. Define the space

$$
X_{\mathrm{div}}:=\left\{(\mathbf{E}, \mathbf{H}, \Phi) \in X \mid \operatorname{div}(\mu \mathbf{H})=0, \mathbf{H} \cdot \nu=0 \text { on } \partial Q, \operatorname{div} \mathbf{E} \in \mathrm{L}^{2}(Q), \Phi=0\right\},
$$

now incorporating Gauss' law of the absence of magnetic monopoles, compare [9]. To prescribe the latter mentioned law, and to obtain solutions of (3.1) that are more regular, we use the space

$$
X_{1}:=\mathcal{D}\left(M_{\text {ext }}\right) \cap X_{\text {div }}
$$

with the norm

$$
\left\|\left(\begin{array}{c}
\mathbf{E} \\
\mathbf{H} \\
\Phi
\end{array}\right)\right\|_{X_{1}}^{2}:=\left\|\left(\begin{array}{c}
\mathbf{E} \\
\mathbf{H} \\
\Phi
\end{array}\right)\right\|_{\mathcal{D}\left(M_{\mathrm{ext}}\right)}^{2}+\|\operatorname{div}(\varepsilon \mathbf{E})\|_{\mathrm{L}^{2}}^{2} .
$$

The space $X_{1}$ is complete, since $M_{\text {ext }}$ is closed on $X$, the divergence operator is closed with its maximal domain, $\varepsilon$ satisfies (1.2), and the normal trace operator is continuous on $\mathrm{H}(\operatorname{div}, Q)$. The following lemma establishes a regularity result for the space $X_{1}$, being important for the regularity analysis of the solutions of (3.1), and eventually also for (1.1).

Lemma 3.4. Let $\varepsilon, \mu, \sigma$ satisfy (1.2). The space $X_{1}$ embeds continuously into $\mathrm{H}^{1}(Q)^{7}$. In particular, the norms on both spaces are equivalent.

Proof. Let $(\mathbf{E}, \mathbf{H}, \Phi) \in X_{1}$. Since the spaces $\mathrm{H}_{N}(\operatorname{curl}$, div, $Q)$ and $\mathrm{H}_{T}($ curl, div, $Q)$ embed into $\mathrm{H}^{1}(Q)^{3}$ and $\mu$ satisfies (1.2), the space $X_{1}$ is a subspace of $\mathrm{H}^{1}(Q)^{7}$.

It remains to show the continuous embedding property. Precondition (1.2) is used below without further notice. The product rule for the divergence operator and Gauss' law imply the identities

$$
\operatorname{div} \mathbf{H}=-\frac{1}{\mu}(\nabla \mu) \cdot \mathbf{H}, \quad \operatorname{div}(\varepsilon \mathbf{E})=\varepsilon \operatorname{div} \mathbf{E}+(\nabla \varepsilon) \cdot \mathbf{E},
$$

resulting with Young's inequality in the relations

$$
\begin{aligned}
0 & =\|\operatorname{div} \mathbf{H}\|_{\mathrm{L}^{2}}^{2}-\left\|\frac{1}{\mu}(\nabla \mu) \cdot \mathbf{H}\right\|_{\mathrm{L}^{2}}^{2} \geq\|\operatorname{div} \mathbf{H}\|_{\mathrm{L}^{2}}^{2}-\frac{\|\nabla \mu\|_{\infty}^{2}}{\delta^{2}}\|\mathbf{H}\|_{\mathrm{L}^{2}}^{2}, \\
\|\operatorname{div}(\varepsilon \mathbf{E})\|_{\mathrm{L}^{2}}^{2} & =\|\varepsilon \operatorname{div} \mathbf{E}\|_{\mathrm{L}^{2}}^{2}+2(\varepsilon \operatorname{div} \mathbf{E},(\nabla \varepsilon) \cdot \mathbf{E})_{\mathrm{L}^{2}}+\|(\nabla \varepsilon) \cdot \mathbf{E}\|_{\mathrm{L}^{2}}^{2} \\
& \geq \frac{1}{2}\|\varepsilon \operatorname{div} \mathbf{E}\|_{\mathrm{L}^{2}}^{2}-2\|\nabla \varepsilon\|_{\mathrm{L}^{\infty}}^{2}\|\mathbf{E}\|_{\mathrm{L}^{2}}^{2} .
\end{aligned}
$$


The desired embedding is now a consequence of $(2.1)$, the norm equivalence of $\|\cdot\|$ and $\|\cdot\|_{\mathrm{L}^{2}}$, and the relations (3.10) and (3.11).

To employ the subspace theory for semigroups on $X_{1}$, it is useful to consider the part of $M_{\text {ext }}$ in $X_{1}$, denoted by $M_{\text {ext,1 }}$. The latter has the domain

$$
\mathcal{D}\left(M_{\text {ext }, 1}\right)=\mathcal{D}\left(M_{\text {ext }}^{2}\right) \cap X_{1} .
$$

Indeed, the inclusion from left to right is clear by definition of $X_{1}$. For the reverse inclusion, let $(\mathbf{E}, \mathbf{H}, \Phi) \in$ $\mathcal{D}\left(M_{\text {ext }}^{2}\right) \cap X_{1}$, and put $(\check{\mathbf{E}}, \check{\mathbf{H}}, \check{\Phi}):=M_{\text {ext }}(\mathbf{E}, \mathbf{H}, \Phi)$. It suffices to show that the latter function is an element of $X_{\text {div }}$. The product rule implies the identity

$$
\operatorname{div} \check{\mathbf{E}}=-\frac{1}{\varepsilon^{2}}(\nabla \varepsilon) \cdot \operatorname{curl} \mathbf{H}-(\nabla \sigma) \cdot \mathbf{E}-\sigma \operatorname{div} \mathbf{E},
$$

so $\operatorname{div} \check{\mathbf{E}}$ is contained in $\mathrm{L}^{2}(Q)$. The field $\check{\mathbf{H}}$ further satisfies the magnetic conditions, since $\Phi=0$ and $(\check{\mathbf{E}}, \check{\mathbf{H}}, \check{\Phi})$ belongs to $\mathcal{D}\left(M_{\text {ext }}\right)$. The identity $\check{\Phi}=0$ finally is a consequence of $(\mathbf{E}, \mathbf{H}, \Phi)$ belonging to $X_{1}$. Altogether, $(\check{\mathbf{E}}, \check{\mathbf{H}}, \check{\Phi})$ is an element of $X_{1}$, and $(\mathbf{E}, \mathbf{H}, \Phi)$ is contained in $\mathcal{D}\left(M_{\text {ext }, 1}\right)$.

Using Proposition 2.3 in [9], we can now show the wellposedness of (3.3) as an evolution equation on $X_{1}$. In view of Lemma 3.4, the extended system consequently has solutions within $\mathrm{H}^{1}(Q)^{7}$, provided that the initial data are chosen appropriately, see Remark 3.6.

Proposition 3.5. The part $M_{\mathrm{ext}, 1}$ of $M_{\mathrm{ext}}$ generates a $\mathrm{C}_{0}$-semigroup $\left(\mathrm{e}^{t M_{\mathrm{ext}, 1}}\right)_{t \geq 0}$ on $X_{1}$, being the restriction of $\left(\mathrm{e}^{t M_{\mathrm{ext}}}\right)_{t \geq 0}$ to $X_{1}$. The semigroup moreover satisfies the bound $\left\|\mathrm{e}^{t M_{\mathrm{ext}, 1}}\right\|_{\mathscr{B}\left(X_{1}\right)} \leq C_{\mathrm{stab}, 1}(1+t)$ for $t \geq 0$ with a uniform constant $C_{\mathrm{stab}, 1}>0$.

Proof. 1) The first goal is to show that the restriction $\left(\left.\mathrm{e}^{t M_{\mathrm{ext}, 1}}\right|_{X_{1}}\right)_{t \geq 0}$ is a strongly continuous semigroup on $X_{1}$. Let $\left(\mathbf{E}_{0}, \mathbf{H}_{0}, \Phi_{0}\right) \in \mathcal{D}\left(M_{\text {ext,1 }}\right)$, and consider the extended Maxwell system (3.1). Denote further the solution of the original system (1.1) with initial data $\left(\mathbf{E}_{0}, \mathbf{H}_{0}\right)$ by $(\mathbf{E}, \mathbf{H})$. The magnetic field $\mathbf{H}$ then satisfies Gauss' law for all $t \geq 0$, and thus $(\mathbf{E}, \mathbf{H}, 0)$ is the unique classical solution of the extended system (3.1). Proposition 3.3 then yields $(\mathbf{E}(t), \mathbf{H}(t), 0)=\mathrm{e}^{t M_{\mathrm{ext}}}\left(\mathbf{E}_{0}, \mathbf{H}_{0}, \Phi_{0}\right)$ for $t \geq 0$. Proposition 2.3 of [9] and (1.2) also imply that the function $\operatorname{div}(\mathbf{E}(t))$ is contained in $\mathrm{L}^{2}(Q)$ for $t \geq 0$. As a result, $(\mathbf{E}(t), \mathbf{H}(t), 0)$ is an element of $X_{1}$, and the family $\left(\mathrm{e}^{t M_{\mathrm{ext}}}\right)_{t \geq 0}$ leaves $X_{1}$ invariant.

To show the desired strong continuity in $X_{1}$, we note that $(\mathbf{E}(t), \mathbf{H}(t), 0) \rightarrow\left(\mathbf{E}_{0}, \mathbf{H}_{0}, \Phi_{0}\right)$ as $t \rightarrow 0$ in the topology of $\mathcal{D}\left(M_{\text {ext }}\right)$ by Proposition 3.3. The statements of Proposition 2.3 in [9] provide the convergence of $\operatorname{div}(\varepsilon \mathbf{E}(t))$ to $\operatorname{div}\left(\varepsilon \mathbf{E}_{0}\right)$ as $t \rightarrow 0$ in $\mathrm{L}^{2}(Q)$. Altogether, $(\mathbf{E}(t), \mathbf{H}(t), 0) \rightarrow\left(\mathbf{E}_{0}, \mathbf{H}_{0}, \Phi_{0}\right)$ in $X_{1}$ as $t \rightarrow 0$. This means that $\left(\left.\mathrm{e}^{t M_{\text {ext }}}\right|_{X_{1}}\right)_{t \geq 0}$ is a $\mathrm{C}_{0}$-semigroup on $X_{1}$ with generator $M_{\text {ext, }}$, see Subsection II.2.3 of [12].

2) In consideration of Proposition 2.3 in [9] and the contractivity of $\left(\mathrm{e}^{t M_{\mathrm{ext}}}\right)_{t \geq 0}$ on $X$, the estimates

$$
\begin{aligned}
\|\operatorname{div}(\varepsilon \mathbf{E}(t))\|_{\mathrm{L}^{2}} & \leq\left\|\operatorname{div}\left(\varepsilon \mathbf{E}_{0}\right)\right\|_{\mathrm{L}^{2}}+\tilde{C} t\left\|\left(\mathbf{E}_{0}, \mathbf{H}_{0}, 0\right)\right\|_{\mathrm{L}^{2}}, \\
\left\|\left(\mathbf{E}(t), \mathbf{H}(t), \Phi_{0}\right)\right\|_{\mathcal{D}\left(M_{\text {ext }}\right)}^{2} & =\left\|\left(\mathbf{E}_{0}, \mathbf{H}_{0}, \Phi_{0}\right)\right\|^{2}+\left\|\mathrm{e}^{t M_{\mathrm{ext}}} M_{\text {ext }}\left(\mathbf{E}_{0}, \mathbf{H}_{0}, \Phi_{0}\right)\right\|^{2} \leq\left\|\left(\mathbf{E}_{0}, \mathbf{H}_{0}, \Phi_{0}\right)\right\|_{\mathcal{D}\left(M_{\text {ext }}\right)}^{2}
\end{aligned}
$$

follow with a uniform constant $\tilde{C}>0$, and yield the asserted linear growth restriction.

The following remark is crucial for the error analysis of the desired exponentially stable scheme.

Remark 3.6. A direct consequence of Proposition 3.5 is the following wellposedness result. Let $\left(\mathbf{E}_{0}, \mathbf{H}_{0}, \Phi_{0}\right) \in$ $\mathcal{D}\left(M_{\text {ext }, 1}\right)$. System (3.1) has a unique classical solution $(\mathbf{E}, \mathbf{H}, \Phi)$, belonging to the space $\mathrm{C}\left([0, \infty), \mathcal{D}\left(M_{\text {ext, } 1)}\right) \cap\right.$ $\mathrm{C}^{1}\left([0, \infty), X_{1}\right)$. In view of Lemma $3.4,(\mathbf{E}(t), \mathbf{H}(t), \Phi(t))$ is an element of $\mathrm{H}^{1}(Q)^{7}$ for every $t \geq 0$, and the proof of Proposition 3.5 further reveals that the mapping $(\mathbf{E}, \mathbf{H})$ is the unique solution of the original system (1.1). $\diamond$

\subsection{Two splittings for the extended Maxwell system}

To obtain a splitting for the extended Maxwell operator, the curl-operator is divided into

$$
\operatorname{curl}=\left(\begin{array}{ccc}
0 & -\partial_{3} & \partial_{2} \\
\partial_{3} & 0 & -\partial_{1} \\
-\partial_{2} & \partial_{1} & 0
\end{array}\right)=\mathscr{C}_{1}-\mathscr{C}_{2}
$$


employing the two operators

$$
\mathscr{C}_{1}:=\left(\begin{array}{ccc}
0 & 0 & \partial_{2} \\
\partial_{3} & 0 & 0 \\
0 & \partial_{1} & 0
\end{array}\right) \quad \text { and } \quad \mathscr{C}_{2}:=\left(\begin{array}{ccc}
0 & \partial_{3} & 0 \\
0 & 0 & \partial_{1} \\
\partial_{2} & 0 & 0
\end{array}\right)
$$

with their maximal domains

$$
\mathcal{D}\left(\mathscr{C}_{j}\right):=\left\{u \in \mathrm{L}^{2}(Q)^{3} \mid \mathscr{C}_{j} u \in \mathrm{L}^{2}(Q)^{3}\right\}, \quad j \in\{1,2\} .
$$

An important relation between $\mathscr{C}_{1}$ and $\mathscr{C}_{2}$ consists in the integration by parts rule

$$
\left(\mathscr{C}_{2} u, v\right)_{\mathrm{L}^{2}}=-\left(u, \mathscr{C}_{1} v\right)_{\mathrm{L}^{2}}
$$

for functions $u=\left(u_{i}\right)_{i=1}^{3} \in \mathcal{D}\left(\mathscr{C}_{2}\right)$ and $v=\left(v_{i}\right)_{i=1}^{3} \in \mathcal{D}\left(\mathscr{C}_{1}\right)$ with

$$
\left(\operatorname{tr}_{\Gamma_{2}} u_{1}\right)\left(\operatorname{tr}_{\Gamma_{2}} v_{3}\right)=0=\left(\operatorname{tr}_{\Gamma_{3}} u_{2}\right)\left(\operatorname{tr}_{\Gamma_{3}} v_{1}\right)=\left(\operatorname{tr}_{\Gamma_{1}} u_{3}\right)\left(\operatorname{tr}_{\Gamma_{1}} v_{2}\right),
$$

compare Section 4.3 of [18]. Note that the corresponding traces are well-defined since both functions have the required partial regularity by definition of $\mathcal{D}\left(\mathscr{C}_{1}\right)$ and $\mathcal{D}\left(\mathscr{C}_{2}\right)$.

The parts associated to the original undamped Maxwell system are then contained in the two operators

$$
A:=\left(\begin{array}{ccc}
0 & \frac{1}{\varepsilon} \mathscr{C}_{1} & 0 \\
\frac{1}{\mu} \mathscr{C}_{2} & 0 & 0 \\
0 & 0 & 0
\end{array}\right) \quad \text { and } \quad B:=\left(\begin{array}{ccc}
0 & -\frac{1}{\varepsilon} \mathscr{C}_{2} & 0 \\
-\frac{1}{\mu} \mathscr{C}_{1} & 0 & 0 \\
0 & 0 & 0
\end{array}\right)
$$

which are equipped with the domains

$$
\begin{aligned}
& \mathcal{D}(A):=\left\{(\mathbf{E}, \mathbf{H}, \Phi) \in X \mid\left(\mathscr{C}_{1} \mathbf{H}, \mathscr{C}_{2} \mathbf{E}, \Phi\right) \in X, \mathbf{E}_{1}=0 \text { on } \Gamma_{2}, \mathbf{E}_{2}=0 \text { on } \Gamma_{3}, \mathbf{E}_{3}=0 \text { on } \Gamma_{1}\right\} \\
& \mathcal{D}(B):=\left\{(\mathbf{E}, \mathbf{H}, \Phi) \in X \mid\left(\mathscr{C}_{2} \mathbf{H}, \mathscr{C}_{1} \mathbf{E}, \Phi\right) \in X, \mathbf{E}_{1}=0 \text { on } \Gamma_{3}, \mathbf{E}_{2}=0 \text { on } \Gamma_{1}, \mathbf{E}_{3}=0 \text { on } \Gamma_{2}\right\}
\end{aligned}
$$

These operators essentially coincide with the ones in [18], and thus Lemma 4.3 from [18] immediately shows the following statement, which is fundamental for the unconditional stability (in the numerical sense) of ADI schemes.

Lemma 3.7. The operators $A$ and $B$ are skewadjoint on $X$. In particular, the operator $(I-\tau L)^{-1}$ is contractive, and the Cayley-Transform

$$
S_{\tau}(L):=\left(I+\frac{\tau}{2} L\right)\left(I-\frac{\tau}{2} L\right)^{-1}
$$

is an isometry on $X$ for $\tau>0$ and $L \in\{A, B\}$.

Let $i \in\{1,2,3\}$, and let $\mathbf{e}_{i} \in \mathbb{R}^{3}$ denote the $i$-th standard unit vector. In order to deal with the ingredients of system (3.1) related to the new artificial quantity $\Phi$, and to preserve the linear complexity of ADI schemes, the operator

$$
D_{i}\left(\begin{array}{c}
\mathbf{E} \\
\mathbf{H} \\
\Phi
\end{array}\right):=\left(\begin{array}{c}
0 \\
-\partial_{i}\left(\frac{1}{\mu} \Phi\right) \mathbf{e}_{i} \\
-\frac{1}{\mu^{2}} \partial_{i}\left(\mu \mathbf{H}_{i}\right)
\end{array}\right)
$$

with domain

$$
\mathcal{D}\left(D_{i}\right):=\mathrm{L}^{2}(Q)^{3} \times\left\{\mathbf{H} \in \mathrm{L}^{2}(Q)^{3} \mid \partial_{i} \mathbf{H}_{i} \in \mathrm{L}^{2}(Q), \mathbf{H}_{i}=0 \text { on } \Gamma_{i}\right\} \times\left\{\Phi \in \mathrm{L}^{2}(Q) \mid \partial_{i} \Phi \in \mathrm{L}^{2}(Q)\right\}
$$

is employed. It is important to note that the boundary condition for the electric field (respectively magnetic field) is distributed onto the domains of the operators $A, B, D_{1}, D_{2}, D_{3}$. This is done in such a way that the imposed partial regularity ensures that all arising traces exist.

These operators at hand, the extended Maxwell operator $M_{\text {ext }}$ is split into the sum

$$
\left(\begin{array}{ccc}
-\sigma & \frac{1}{\varepsilon} \operatorname{curl} & 0 \\
-\frac{1}{\mu} \operatorname{curl} & 0 & -\nabla\left(\frac{1}{\mu} \cdot\right) \\
0 & -\frac{1}{\mu^{2}} \operatorname{div}(\mu \cdot) & -\eta
\end{array}\right)=\left(\begin{array}{ccc}
-\sigma & 0 & 0 \\
0 & 0 & 0 \\
0 & 0 & -\eta
\end{array}\right)+A+B+D_{1}+D_{2}+D_{3}
$$

on the intersection $\mathcal{D}(A) \cap \mathcal{D}(B) \cap \mathcal{D}\left(D_{1}\right) \cap \mathcal{D}\left(D_{2}\right) \cap \mathcal{D}\left(D_{3}\right) \subseteq \mathcal{D}\left(M_{\text {ext }}\right)$. The next lemma establishes the statements of Lemma 3.7 for the splitting operators $D_{i}$, and will frequently be used in our arguments. 
Lemma 3.8. Let $i \in\{1,2,3\}$. The operator $D_{i}$ is skewadjoint on $X$, and consequently the Cayley-Transform $S_{\tau}\left(D_{i}\right):=\left(I+\frac{\tau}{2} D_{i}\right)\left(I-\frac{\tau}{2} D_{i}\right)^{-1}$ is an isometry on $X$ for $\tau>0$.

Proof. The operator $D_{i}$ is clearly densely defined on $X$. The closedness of $D_{i}$ further is an immediate consequence of the closedness of the weak derivative $\partial_{i}$ with respect to its maximal domain $\mathcal{D}\left(\partial_{i}\right)$ in $\mathrm{L}^{2}(Q)$, the boundedness of the trace operator $\operatorname{tr}_{\Gamma_{i}}$ on $\mathcal{D}\left(\partial_{i}\right)$, and the assumption (1.2) on $\mu$.

We next show that $D_{i}$ is skewsymmetric. Let therefore $\left(\mathbf{E}^{1}, \mathbf{H}^{1}, \Phi^{1}\right),\left(\mathbf{E}^{2}, \mathbf{H}^{2}, \Phi^{2}\right) \in \mathcal{D}\left(D_{i}\right)$. In view of the boundary conditions for $\mathbf{H}_{i}^{1}$ and $\mathbf{H}_{i}^{2}$, an integration by parts leads to the identities

$$
\begin{aligned}
\left(D_{i}\left(\begin{array}{c}
\mathbf{E}^{1} \\
\mathbf{H}^{1} \\
\Phi^{1}
\end{array}\right),\left(\begin{array}{c}
\mathbf{E}^{2} \\
\mathbf{H}^{2} \\
\Phi^{2}
\end{array}\right)\right) & =-\int_{Q}\left(\mu \partial_{i}\left(\frac{1}{\mu} \Phi^{1}\right) \mathbf{H}_{i}^{2}+\frac{1}{\mu} \partial_{i}\left(\mu \mathbf{H}_{i}^{1}\right) \Phi^{2}\right) \mathrm{d} x=\int_{Q}\left(\frac{1}{\mu} \Phi^{1} \partial_{i}\left(\mu \mathbf{H}_{i}^{2}\right)+\mu \mathbf{H}_{i}^{1} \partial_{i}\left(\frac{1}{\mu} \Phi^{2}\right)\right) \mathrm{d} x \\
& =-\left(\left(\begin{array}{c}
\mathbf{E}^{1} \\
\mathbf{H}^{1} \\
\Phi^{1}
\end{array}\right), D_{i}\left(\begin{array}{c}
\mathbf{E}^{2} \\
\mathbf{H}^{2} \\
\Phi^{2}
\end{array}\right)\right)
\end{aligned}
$$

meaning that $D_{i}$ is skewsymmetric and in particular dissipative.

It remains to show that $D_{i}^{*}$ is extended by $-D_{i}$. Let $(\tilde{\mathbf{E}}, \tilde{\mathbf{H}}, \tilde{\Phi}) \in \mathcal{D}\left(D_{i}^{*}\right)$, and abbreviate $(\hat{\mathbf{E}}, \hat{\mathbf{H}}, \hat{\Phi}):=$ $D_{i}^{*}(\tilde{\mathbf{E}}, \tilde{\mathbf{H}}, \tilde{\Phi})$. Since $D_{i}^{*}$ is adjoint to $D_{i}$, the formula

$$
\left(\left(\begin{array}{c}
\mathbf{E} \\
\mathbf{H} \\
\Phi
\end{array}\right),\left(\begin{array}{c}
\hat{\mathbf{E}} \\
\hat{\mathbf{H}} \\
\hat{\Phi}
\end{array}\right)\right)=\left(D_{i}\left(\begin{array}{c}
\mathbf{E} \\
\mathbf{H} \\
\Phi
\end{array}\right),\left(\begin{array}{c}
\tilde{\mathbf{E}} \\
\tilde{\mathbf{H}} \\
\tilde{\Phi}
\end{array}\right)\right)=-\int_{Q}\left(\mu \partial_{i}\left(\frac{1}{\mu} \Phi\right) \tilde{\mathbf{H}}_{i}+\frac{1}{\mu} \partial_{i}\left(\mu \mathbf{H}_{i}\right) \tilde{\Phi}\right) \mathrm{d} x
$$

follows for every vector $(\mathbf{E}, \mathbf{H}, \Phi) \in \mathcal{D}\left(D_{i}\right)$. Choosing $\mathbf{E}=\mathbf{H}=0$, we conclude the relation

$$
-\int_{Q} \mu \partial_{i}\left(\frac{1}{\mu} \Phi\right) \tilde{\mathbf{H}}_{i} \mathrm{~d} x=\int_{Q} \mu \Phi \hat{\Phi} \mathrm{d} x
$$

for every function $\Phi \in \mathrm{L}^{2}(Q)$ with $\partial_{i} \Phi \in \mathrm{L}^{2}(Q)$. The function $\partial_{i}\left(\mu \tilde{\mathbf{H}}_{i}\right)$ thus belongs to $\mathrm{L}^{2}(Q)$, and satisfies $\frac{1}{\mu^{2}} \partial_{i}\left(\mu \tilde{\mathbf{H}}_{i}\right)=\hat{\Phi}$ as well as $\mu \tilde{\mathbf{H}}_{i}=0$ on $\Gamma_{i}$. Take now $\Phi=0, \mathbf{E}=0$, and $\mathbf{H}_{j}=0$ for $j \neq i$ in (3.15). In this way the equation

$$
-\int_{Q} \frac{1}{\mu} \partial_{i}\left(\mu \mathbf{H}_{i}\right) \tilde{\Phi} \mathrm{d} x=\int_{Q} \mu \mathbf{H}_{i} \hat{\mathbf{H}}_{i} \mathrm{~d} x
$$

is derived for all $\mathbf{H}_{i} \in \mathrm{L}^{2}(Q)$ with $\partial_{i} \mathbf{H}_{i} \in \mathrm{L}^{2}(Q)$ and $\mathbf{H}_{i}=0$ on $\Gamma_{i}$. As a result, $\partial_{i}\left(\frac{1}{\mu} \tilde{\Phi}\right)$ is contained in $\mathrm{L}^{2}(Q)$ and $\hat{\mathbf{H}}_{i}=\partial_{i}\left(\frac{1}{\mu} \tilde{\Phi}\right)$. The assumptions (1.2) on $\mu$ finally imply that $(\tilde{\mathbf{E}}, \tilde{\mathbf{H}}, \tilde{\Phi})$ is an element of $\mathcal{D}\left(D_{i}\right)$, and hence $D_{i}$ is skewadjoint.

By means of Lemmas 3.7 and 3.8, we can introduce an important ingredient of the exponentially stable ADI scheme, namely artificial damping. We therefore employ an operator from [14]. Let $\tau \in\left(0, \frac{1}{2}\right)$ and $L \in$ $\left\{A, B, D_{1}, D_{2}, D_{3}\right\}$, and consider the operator $I-\frac{\tau^{3}}{4} L^{2}\left(I-\frac{\tau^{2}}{4} L^{2}\right)^{-1}$. This mapping is clearly well-defined since the squared operator $L^{2}$ is negative by Lemmas 3.7 and 3.8. The same results further imply that the inverse

$$
V_{\tau}(L):=\left(I-\frac{\tau^{3}}{4} L^{2}\left(I-\frac{\tau^{2}}{4} L^{2}\right)^{-1}\right)^{-1}
$$

is bounded on $X$, as the linear operator $\frac{\tau^{3}}{4} L^{2}\left(I-\frac{\tau^{2}}{4} L^{2}\right)^{-1}=\tau\left(-I+\left(I-\frac{\tau}{2} L\right)^{-1}\left(I+\frac{\tau}{2} L\right)^{-1}\right)$ has norm less than one.

The established framework now allows to formulate the following two schemes for the approximation of the extended Maxwell system (3.1). Let $n \in \mathbb{N}_{0}$, and let $\tau>0$ denote the time step size. We first consider the undamped case, where $\sigma=\eta=0$ and the energy of system (3.1) is conserved. It is then natural to approximate 
the solution of (3.1) by a scheme that is also energy conserving. Beginning with initial data $\left(\mathbf{E}_{c}^{0}, \mathbf{H}_{c}^{0}, \Phi_{c}^{0}\right)$, the solution $(\mathbf{E}, \mathbf{H}, \Phi)$ of $(3.1)$ at time $t=(n+1) \tau$ is approximated by computing

$$
\left(\begin{array}{c}
\mathbf{E}_{c}^{n+1} \\
\mathbf{H}_{c}^{n+1} \\
\Phi_{c}^{n+1}
\end{array}\right)=S_{\tau}\left(D_{3}\right) S_{\tau}\left(D_{2}\right) S_{\tau}\left(D_{1}\right) S_{\tau}(B) S_{\tau}(A)\left(\begin{array}{c}
\mathbf{E}_{c}^{n} \\
\mathbf{H}_{c}^{n} \\
\Phi_{c}^{n}
\end{array}\right)
$$

where $S_{\tau}(L)=\left(I+\frac{\tau}{2} L\right)\left(I-\frac{\tau}{2} L\right)^{-1}$ again denotes the Cayley-Transform for $L \in\left\{A, B, D_{1}, D_{2}, D_{3}\right\}$, and the subscript $c$ stresses that the conservative undamped problem is considered. For the Maxwell system (1.1), a similar scheme was originally proposed in [5]. Here it is mainly used to deduce the uniform exponential stability of the succeeding scheme. In view of Lemmas 3.7 and 3.8, it is clear that (3.17) defines an energy conserving method.

Consider now the damped case, i.e., let $\sigma, \eta \in W^{1, \infty}(Q)$ with $\sigma, \eta \geq \delta>0$. Starting with an initial value $\left(\mathbf{E}^{0}, \mathbf{H}^{0}, \Phi^{0}\right)$, the solution of $(3.1)$ at time $t=(n+1) \tau$ is approximated via

$$
\left(\begin{array}{l}
\mathbf{E}^{n+1} \\
\mathbf{H}^{n+1} \\
\Phi^{n+1}
\end{array}\right)=\left(\begin{array}{ccc}
\mathrm{e}^{-\tau \sigma} & 0 & 0 \\
0 & I & 0 \\
0 & 0 & \mathrm{e}^{-\tau \eta}
\end{array}\right) \prod_{i=1}^{3}\left(S_{\tau}\left(D_{i}\right) V_{\tau}\left(D_{i}\right)\right) S_{\tau}(B) V_{\tau}(B) S_{\tau}(A) V_{\tau}(A)\left(\begin{array}{c}
\mathbf{E}^{n} \\
\mathbf{H}^{n} \\
\Phi^{n}
\end{array}\right) .
$$

The product sign here means that the respective operators are concatenated such that their indices decrease from left to right. During the error analysis, we will assume that the start value $\left(\mathbf{E}^{0}, \mathbf{H}^{0}, \Phi^{0}\right)$ of $(3.18)$ and the initial data $\left(\mathbf{E}_{0}, \mathbf{H}_{0}, \Phi_{0}\right)$ of (3.1) coincide. To state the main result about uniform exponential stability of (3.18), we define the number

$$
\kappa_{Y}:=\frac{\|\nabla \varepsilon\|_{\infty}+2\|\nabla \mu\|_{\infty}\left(1+\frac{\|\nabla \mu\|_{\infty}}{\delta}\right)}{2 \delta^{2}}+C_{S} \frac{1+\delta}{\delta^{3}}\left(\left\|\partial^{2} \mu\right\|_{L^{3}}+\frac{2}{\delta}\|\nabla \mu\|_{\infty}^{2}\right)\|\mu\|_{\infty}^{1 / 2} \geq 0,
$$

employing the Sobolev constant $C_{S}>0$ from the embedding $\mathrm{H}^{1}(Q) \hookrightarrow \mathrm{L}^{6}(Q)$, the Hermitian $\partial^{2} \mu$ of $\mu$, and the number $\delta$ from (1.2).

Theorem 3.9. Let $\varepsilon, \mu, \sigma, \eta$ satisfy (1.2) and (3.2), and $\zeta \in(0,1)$ be fixed. Let further $\left(\boldsymbol{E}^{n}, \boldsymbol{H}^{n}\right.$, $\left.\Phi^{n}\right)$ be the iterates of (3.18) for initial data $\left(\boldsymbol{E}^{0}, \boldsymbol{H}^{0}, \Phi^{0}\right) \in \mathrm{L}^{2}(Q)^{7}$. There are constants $K, \omega>0$ with

$$
\left\|\left(\begin{array}{l}
\boldsymbol{E}^{n} \\
\boldsymbol{H}^{n} \\
\Phi^{n}
\end{array}\right)\right\|^{2} \leq K \mathrm{e}^{-\omega \tau n}\left\|\left(\begin{array}{c}
\boldsymbol{E}^{0} \\
\boldsymbol{H}^{0} \\
\Phi^{0}
\end{array}\right)\right\|^{2}, \quad n \in \mathbb{N},
$$

for all step sizes $\tau \in\left(0, \zeta \cdot \min \left\{\frac{\sqrt{2}}{\kappa_{Y}}, \frac{1}{2}\right\}\right]$. The numbers $K, \omega$ depend only on $\varepsilon, \mu, \sigma, \eta, \zeta$ and $Q$.

Theorem 3.9 is proved in Section 5, and the proof is heavily based on an observability inequality, being deduced in Section 4 for scheme (3.17).

Remark 3.10. 1) Let $\left(\mathbf{E}^{0}, \mathbf{H}^{0}, \Phi^{0}\right) \in X_{1}$. Theorem 6.5 and Remark 3.6 on the one hand imply that the approximations $\left(\mathbf{E}^{n}, \mathbf{H}^{n}, \Phi^{n}\right)$ converge to the solution $(\mathbf{E}, \mathbf{H}, 0)$ of the extended system (3.1) with (E, H) solving the original problem (1.1). Theorem 3.9 on the other hand yields in this situation that the energy of the iterates $\left(\mathbf{E}^{n}, \mathbf{H}^{n}\right)$ decays in a uniform exponential way. As a result, Theorem 3.9 serves as the time-discrete counterpart of the exponential stability result for (1.1). Another consequence of this theorem is the unconditional (numerical) stability of the damped scheme (3.18) in $X$.

2) The restriction on the time step size is due to technical reasons. The first condition $\tau<\frac{\sqrt{2}}{\kappa_{Y}}$ arises when we consider the splitting scheme in a subspace $Y$ of $\mathrm{H}^{1}(Q)^{7}$, see Subsection 3.3. The second upper bound on the time step size is employed during the proof of the observability estimate to account for boundary terms in a discrete analogue of integration by parts.

\subsection{Analysis of the splitting operators}

The proof of an observability estimate for (3.17) requires a subspace of $X$ consisting of $\mathrm{H}^{1}$-regular functions with appropriate boundary conditions. In analogy to [9], the space

$$
Y:=\left\{(\mathbf{E}, \mathbf{H}, \Phi) \in \mathrm{H}^{1}(Q)^{7} \mid \mathbf{E} \times \nu=0, \mathbf{H} \cdot \nu=0 \text { on } \partial Q\right\}
$$


is employed with the inner product

$$
\left(\left(\begin{array}{c}
\mathbf{E} \\
\mathbf{H} \\
\Phi
\end{array}\right),\left(\begin{array}{c}
\tilde{\mathbf{E}} \\
\tilde{\mathbf{H}} \\
\tilde{\Phi}
\end{array}\right)\right)_{Y}:=\left(\left(\begin{array}{c}
\mathbf{E} \\
\mathbf{H} \\
\Phi
\end{array}\right),\left(\begin{array}{c}
\tilde{\mathbf{E}} \\
\tilde{\mathbf{H}} \\
\tilde{\Phi}
\end{array}\right)\right)+\sum_{j=1}^{3} \int_{Q}\left(\varepsilon\left(\partial_{j} \mathbf{E}\right) \cdot\left(\partial_{j} \tilde{\mathbf{E}}\right)+\mu\left(\partial_{j} \mathbf{H}\right) \cdot\left(\partial_{j} \tilde{\mathbf{H}}\right)+\mu\left(\partial_{j} \Phi\right)\left(\partial_{j} \tilde{\Phi}\right)\right) \mathrm{d} x,
$$

which induces the norm $\|\cdot\|_{Y}$. Note that $X_{1}$ is a subspace of $Y$, and that $Y$ is contained in the domains of all splitting operators. It will be crucial to ensure that the schemes (3.17) and (3.18) yield iterates within $Y$ if the initial data are chosen appropriately. Therefore, the parts $A_{Y}, B_{Y}$, and $D_{i, Y}$ of $A, B$, and $D_{i}$ in $Y$ are considered for $i \in\{1,2,3\}$. In view of $(3.13),(3.14)$, and (1.2), the identities

$$
\begin{aligned}
\mathcal{D}\left(A_{Y}\right) & :=\left\{(\mathbf{E}, \mathbf{H}, \Phi) \in Y \mid \mathscr{C}_{1} \mathbf{H}, \mathscr{C}_{2} \mathbf{E} \in \mathrm{H}^{1}(Q)^{3},\left(\mathscr{C}_{1} \mathbf{H}\right) \times \nu=0,\left(\mathscr{C}_{2} \mathbf{E}\right) \cdot \nu=0 \text { on } \partial Q\right\}, \\
\mathcal{D}\left(B_{Y}\right) & :=\left\{(\mathbf{E}, \mathbf{H}, \Phi) \in Y \mid \mathscr{C}_{2} \mathbf{H}, \mathscr{C}_{1} \mathbf{E} \in \mathrm{H}^{1}(Q)^{3},\left(\mathscr{C}_{2} \mathbf{H}\right) \times \nu=0,\left(\mathscr{C}_{1} \mathbf{E}\right) \cdot \nu=0 \text { on } \partial Q\right\}, \\
\mathcal{D}\left(D_{i, Y}\right) & :=\left\{(\mathbf{E}, \mathbf{H}, \Phi) \in Y \mid \partial_{i} \mathbf{H}_{i} \in \mathrm{H}^{1}(Q), \partial_{i} \Phi \in \mathrm{H}^{1}(Q), \partial_{i} \Phi=0 \text { on } \Gamma_{i}\right\}
\end{aligned}
$$

readily follow. The operators $A_{Y}$ and $B_{Y}$ are essentially analyzed by Eilinghoff and Schnaubelt in [9], and their Proposition 3.6 yields the following crucial result.

Lemma 3.11. Let $L \in\{ \pm A, \pm B\}$. The part $L_{Y}$ of $L$ in $Y$ has $\left(\kappa_{Y}, \infty\right)$ in its resolvent set, generates a strongly continuous semigroup, and $\left.(I-\tau L)^{-1}\right|_{Y}$ coincides with $\left(I-\tau L_{Y}\right)^{-1}$. The latter operator satisfies the estimate

$$
\left\|\left(I-\tau L_{Y}\right)^{-1}\right\|_{\mathscr{B}(Y)} \leq \frac{1}{1-\tau \kappa_{Y}}, \quad \tau \in\left(0, \frac{1}{\kappa_{Y}}\right) .
$$

There moreover is a constant $\tau_{0} \in\left(0, \frac{1}{2 \kappa_{Y}}\right)$, depending only on $\kappa_{Y}$, such that the corresponding Cayley-Transform $S_{\tau}\left(L_{Y}\right)$ can be bounded by

$$
\left\|S_{\tau}\left(L_{Y}\right)\right\|_{\mathscr{B}(Y)} \leq \mathrm{e}^{3 \kappa_{Y} \tau}, \quad \tau \in\left(0, \tau_{0}\right]
$$

The major goal of this subsection now consists in the deduction of Lemma 3.11 for the remaining splitting operators. This will mainly be done in the next three lemmas by transferring the arguments of the proofs for Lemmas 3.3-3.5 in [9] to the mappings $D_{i}$.

Lemma 3.12. Let $i \in\{1,2,3\}$. The operator $D_{i, Y}$ is closed and densely defined in $Y$.

Proof. The operator $D_{i, Y}$ is closed in $Y$ as the part of a closed operator. It remains to approximate a fixed vector $(\mathbf{E}, \mathbf{H}, \Phi) \in Y$ by a sequence in $\mathcal{D}\left(D_{i, Y}\right)$. Since only the components $\mathbf{H}_{i}$ and $\Phi$ need to be considered, we first look for functions $\mathbf{H}_{i}^{n}$ in $\mathrm{H}^{1}(Q)$ with $\partial_{i} \mathbf{H}_{i}^{n} \in \mathrm{H}^{1}(Q)$ and $\mathbf{H}_{i}^{n}=0$ on $\Gamma_{i}$, that converge to $\mathbf{H}_{i}$ in $\mathrm{H}^{1}(Q)$. The arguments in part 2 of Lemma 3.3 in [9] provide such functions. Adapting part 3 of the proof for Lemma 3.3 in [9], we further obtain mappings $\Phi^{n}$ in $\mathrm{H}^{1}(Q)$ with $\partial_{i} \Phi^{n} \in \mathrm{H}^{1}(Q), \partial_{i} \Phi^{n}=0$ on $\Gamma_{i}$, and $\Phi^{n} \rightarrow \Phi$ in $\mathrm{H}^{1}(Q)$. Let finally $\mathbf{H}_{j}^{n}:=\mathbf{H}_{j}$ and $\mathbf{E}^{n}:=\mathbf{E}$ for $n \in \mathbb{N}$ and $j \in\{1,2,3\} \backslash\{i\}$. Employing (1.2), $\left(\mathbf{E}^{n}, \mathbf{H}^{n}, \Phi^{n}\right)_{n}$ is the desired approximating sequence in $\mathcal{D}\left(D_{i, Y}\right)$.

The higher regularity assumption (1.2) for $\mu$ is also essential in the proof of the next lemma.

Lemma 3.13. Let $i \in\{1,2,3\}$. The operator $\pm D_{i, Y}-\kappa_{Y} I$ is dissipative in $X$.

Proof. We only consider the operator $D_{i, Y}$. Let $(\mathbf{E}, \mathbf{H}, \Phi) \in \mathcal{D}\left(D_{i, Y}\right)$. In view of the boundary conditions for $\mathbf{H}_{i}$, Lemma 2.1 in [9] shows that $\partial_{j} \mathbf{H}_{i}=0$ on $\Gamma_{i}$ for $j \neq i$. Employing now also the boundary condition $\partial_{i} \Phi=0$ on $\Gamma_{i}$ in an integration by parts, the identities

$$
\int_{Q}\left(\left(\partial_{j} \partial_{i} \Phi\right)\left(\partial_{j} \mathbf{H}_{i}\right)+\left(\partial_{j} \partial_{i} \mathbf{H}_{i}\right)\left(\partial_{j} \Phi\right)\right) \mathrm{d} x=\int_{Q}\left(-\left(\partial_{j} \Phi\right)\left(\partial_{j} \partial_{i} \mathbf{H}_{i}\right)+\left(\partial_{j} \partial_{i} \mathbf{H}_{i}\right) \partial_{j} \Phi\right) \mathrm{d} x=0
$$

are derived for $j \in\{1,2,3\}$. Since $D_{i}$ is skewadjoint on $X$, we further conclude

$$
\left(D_{i}\left(\begin{array}{c}
\mathbf{E} \\
\mathbf{H} \\
\Phi
\end{array}\right),\left(\begin{array}{c}
\mathbf{E} \\
\mathbf{H} \\
\Phi
\end{array}\right)\right)_{Y}=-\sum_{j=1}^{3} \int_{Q}\left(\mu\left(\partial_{j} \partial_{i} \frac{1}{\mu} \Phi\right) \partial_{j} \mathbf{H}_{i}+\mu\left(\partial_{j} \frac{1}{\mu^{2}} \partial_{i} \mu \mathbf{H}_{i}\right) \partial_{j} \Phi\right) \mathrm{d} x
$$




$$
\begin{aligned}
= & -\sum_{j=1}^{3} \int_{Q}\left(\left(\partial_{j} \partial_{i} \Phi\right) \partial_{j} \mathbf{H}_{i}+\left(\partial_{j} \partial_{i} \mathbf{H}_{i}\right) \partial_{j} \Phi\right) \mathrm{d} x+\sum_{j=1}^{3} \int_{Q}\left(\frac{\partial_{j} \mu}{\mu}\left(\partial_{i} \Phi\right) \partial_{j} \mathbf{H}_{i}+\frac{\partial_{j} \mu}{\mu}\left(\partial_{i} \mathbf{H}_{i}\right) \partial_{j} \Phi\right) \mathrm{d} x \\
& -\sum_{j=1}^{3} \int_{Q}\left(\mu\left(\partial_{j} \partial_{i} \frac{1}{\mu}\right) \Phi \partial_{j} \mathbf{H}_{i}+\mu\left(\partial_{j} \frac{\partial_{i} \mu}{\mu^{2}}\right) \mathbf{H}_{i} \partial_{j} \Phi-2\left(\partial_{i} \mu\right) \frac{\partial_{j} \mu}{\mu^{2}} \mathbf{H}_{i} \partial_{j} \Phi\right) \mathrm{d} x .
\end{aligned}
$$

The above calculation implies that the first integral on the right hand side of (3.19) vanishes. By means of the assumption (1.2) on $\mu$, the second integral and the last expression in the third integral are also easily estimated from above by the norm of $(\mathbf{E}, \mathbf{H}, \Phi)$ in $Y$. For the two remaining terms in the third integral, we use Sobolev's embedding $\mathrm{H}^{1}(Q) \hookrightarrow \mathrm{L}^{6}(Q)$ with constant $C_{S}>0$. Applying also Hölder's inequality, the estimates

$$
\begin{aligned}
\left|\sum_{j=1}^{3} \int_{Q} \mu\left(\partial_{j} \partial_{i} \frac{1}{\mu}\right) \Phi \partial_{j} \mathbf{H}_{i} \mathrm{~d} x\right| & \leq\left(\frac{1}{\delta^{2}}\left\|\partial^{2} \mu\right\|_{\mathrm{L}^{3}}+\frac{2}{\delta^{3}}\|\nabla \mu\|_{\infty}^{2}\right)\|\mu\|_{\infty}^{1 / 2}\left\|\sqrt{\mu} \nabla \mathbf{H}_{i}\right\|_{\mathrm{L}^{2}}\|\Phi\|_{\mathrm{L}^{6}} \\
& \leq C_{S} \frac{1+\delta}{2 \delta^{3}}\left(\left\|\partial^{2} \mu\right\|_{\mathrm{L}^{3}}+\frac{2}{\delta}\|\nabla \mu\|_{\infty}^{2}\right)\|\mu\|_{\infty}^{1 / 2}\left\|\left(\begin{array}{c}
\mathbf{E} \\
\mathbf{H} \\
\Phi
\end{array}\right)\right\|_{Y}^{2}
\end{aligned}
$$

are obtained. Recall that $\partial^{2} \mu$ denotes the Hessian of $\mu$. Treating the last remaining term in the same way, we altogether arrive at the desired relation

$$
\left(D_{i}\left(\begin{array}{c}
\mathbf{E} \\
\mathbf{H} \\
\Phi
\end{array}\right),\left(\begin{array}{c}
\mathbf{E} \\
\mathbf{H} \\
\Phi
\end{array}\right)\right)_{Y} \leq \kappa_{Y}\left\|\left(\begin{array}{c}
\mathbf{E} \\
\mathbf{H} \\
\Phi
\end{array}\right)\right\|_{Y}^{2}
$$

Lemma 3.14. Let $i \in\{1,2,3\}$. The operator $\left(1+\kappa_{Y}\right) I \pm D_{i, Y}$ has dense range in $Y$.

Proof. 1) We only treat the case $i=1$. It again suffices to consider only the operator $\left(1+\kappa_{Y}\right) I-D_{1, Y}$, and to show that its range contains the domain $\mathcal{D}\left(D_{1, Y}\right)$. For that purpose, let $(\check{\mathbf{E}}, \check{\mathbf{H}}, \check{\Phi}) \in \mathcal{D}\left(D_{1, Y}\right)$. The goal is to find a function $(\mathbf{E}, \mathbf{H}, \Phi) \in \mathcal{D}\left(D_{1, Y}\right)$ with $\left(\left(1+\kappa_{Y}\right) I-D_{1, Y}\right)(\mathbf{E}, \mathbf{H}, \Phi)=(\check{\mathbf{E}}, \check{\mathbf{H}}, \check{\Phi})$, meaning

$$
\begin{aligned}
\left(1+\kappa_{Y}\right) \mathbf{E} & =\check{\mathbf{E}}, \\
\left(1+\kappa_{Y}\right) \mathbf{H}_{j} & =\check{\mathbf{H}}_{j} \quad \text { for } j \in\{2,3\}, \\
\left(1+\kappa_{Y}\right) \mathbf{H}_{1}+\partial_{1}\left(\frac{1}{\mu} \Phi\right) & =\check{\mathbf{H}}_{1}, \\
\left(1+\kappa_{Y}\right) \Phi+\frac{1}{\mu^{2}} \partial_{1}\left(\mu \mathbf{H}_{1}\right) & =\check{\Phi} .
\end{aligned}
$$

Formally plugging the fourth line into the third leads to the identity

$$
\mathbf{H}_{1}-\frac{1}{\left(1+\kappa_{Y}\right)^{2}} \partial_{1}\left(\frac{1}{\mu^{3}} \partial_{1} \mu \mathbf{H}_{1}\right)=\frac{1}{1+\kappa_{Y}} \check{\mathbf{H}}_{1}-\frac{1}{\left(1+\kappa_{Y}\right)^{2}} \partial_{1}\left(\frac{1}{\mu} \check{\Phi}\right)=: h_{1},
$$

being equivalent to the relation

$$
\frac{1}{\mu} \stackrel{\circ}{H}_{1}-\frac{1}{\left(1+\kappa_{Y}\right)^{2}} \partial_{1, \mu}^{2} \stackrel{\circ}{\mathbf{H}}_{1}=h_{1},
$$

where $\stackrel{\circ}{\mathbf{H}}_{1}:=\mu \mathbf{H}_{1}$, and the operator $\partial_{1, \mu}^{2}:=\partial_{1} \frac{1}{\mu^{3}} \partial_{1}$ is equipped with the domain

$$
\mathcal{D}\left(\partial_{1, \mu}^{2}\right):=\left\{u \in \mathrm{L}^{2}(Q) \mid \partial_{1} u, \partial_{1}^{2} u \in \mathrm{L}^{2}(Q), u=0 \text { on } \Gamma_{1}\right\} .
$$

Note that the function $h_{1}$ belongs to $\mathrm{H}^{1}(Q)$ since $(\check{\mathbf{E}}, \check{\mathbf{H}}, \check{\Phi})$ is contained in $\mathcal{D}\left(D_{1, Y}\right)$. The domain

$$
\mathcal{D}\left(\partial_{1}\right):=\left\{u \in \mathrm{L}^{2}(Q) \mid \partial_{1} u \in \mathrm{L}^{2}(Q), u=0 \text { on } \Gamma_{1}\right\}
$$

will also be employed. 
2) The left hand side of (3.21) is associated with the operator

$$
L_{1} w:=\frac{1}{\mu} w-\frac{1}{\left(1+\kappa_{Y}\right)^{2}} \partial_{1, \mu}^{2} w, \quad w \in \mathcal{D}\left(\partial_{1, \mu}^{2}\right)=: \mathcal{D}\left(L_{1}\right) .
$$

As in the proof of Lemma 4.3 in [18], the Lax-Milgram Lemma yields a unique function $w \in \mathcal{D}\left(L_{1}\right)$ satisfying $L_{1} w=$ $h_{1}$. We now choose $\mathbf{E}=\frac{1}{1+\kappa_{Y}} \check{\mathbf{E}}, \mathbf{H}_{1}=\frac{1}{\mu} \stackrel{\circ}{1}_{1}:=\frac{1}{\mu} w, \mathbf{H}_{j}=\frac{1}{1+\kappa_{Y}} \check{\mathbf{H}}_{j}$ for $j \in\{2,3\}$ and $\Phi:=\frac{1}{1+\kappa_{Y}}\left(\check{\Phi}-\frac{1}{\mu^{2}} \partial_{1}\left(\mu \mathbf{H}_{1}\right)\right)$. It only remains to show that the vector $(\mathbf{E}, \mathbf{H}, \Phi)$ belongs to $\mathcal{D}\left(D_{1, Y}\right)$, i.e., that $\partial_{1} \mathbf{H}_{1}$ and $\partial_{1} \Phi$ are elements of $\mathrm{H}^{1}(Q)$ and $\partial_{1} \Phi=0$ on $\Gamma_{1}$.

Let $k \in\{2,3\}$ and $\varphi \in \mathrm{H}_{0}^{2}(Q)$. The assumption (1.2) on $\mu$ implies that $\partial_{1}^{2} w$ belongs to $\mathrm{L}^{2}(Q)$, and thus $\partial_{1, \mu}^{2} \partial_{k} w=\partial_{k} \partial_{1, \mu}^{2} w-\partial_{1}\left(\partial_{k} \frac{1}{\mu^{3}}\right) \partial_{1} w$ is an element of $\mathrm{H}^{-1}(Q)$. The same is true for the distribution $\partial_{1} \partial_{k} w$. The relation $L_{1} w=h_{1}$ and integration by parts thus imply the equations

$$
\begin{aligned}
\left\langle\frac{1}{\mu} \partial_{k} w-\frac{1}{\left(1+\kappa_{Y}\right)^{2}} \partial_{1, \mu}^{2} \partial_{k} w, \varphi\right\rangle_{\mathrm{H}^{-2} \times \mathrm{H}_{0}^{2}}=-\int_{Q}\left(w \partial_{k}\left(\frac{1}{\mu} \varphi\right)+\frac{1}{\left(1+\kappa_{Y}\right)^{2}}\left(\partial_{1} w\right) \partial_{k}\left(\frac{1}{\mu^{3}} \partial_{1} \varphi\right)\right) \mathrm{d} x \\
=-\int_{Q}\left(h_{1} \partial_{k} \varphi+\frac{1}{\left(1+\kappa_{Y}\right)^{2}}\left(\partial_{1, \mu}^{2} w\right) \partial_{k} \varphi+w \varphi \partial_{k} \frac{1}{\mu}+\frac{1}{\left(1+\kappa_{Y}\right)^{2}}\left(\partial_{1} w\right) \partial_{k}\left(\frac{1}{\mu^{3}} \partial_{1} \varphi\right)\right) \mathrm{d} x \\
=-\int_{Q}\left(h_{1} \partial_{k} \varphi+\frac{1}{\left(1+\kappa_{Y}\right)^{2}}\left(\partial_{1} w\right)\left(\partial_{1} \varphi\right) \partial_{k} \frac{1}{\mu^{3}}+w \varphi \partial_{k} \frac{1}{\mu}\right) \mathrm{d} x \\
=\int_{Q}\left(\partial_{k} h_{1}\right) \varphi-\left(\partial_{k} \frac{1}{\mu}\right) w \varphi \mathrm{d} x+\frac{1}{\left(1+\kappa_{Y}\right)^{2}}\left\langle\partial_{1}\left(\left(\partial_{k} \frac{1}{\mu^{3}}\right) \partial_{1} w\right), \varphi\right\rangle_{\mathcal{D}\left(\partial_{1}\right)^{*} \times \mathcal{D}\left(\partial_{1}\right)}
\end{aligned}
$$

Since $\mathrm{H}_{0}^{2}(Q)$ is dense in $\mathcal{D}\left(\partial_{1}\right)$, w satisfies the formula

$$
\frac{1}{\mu} \partial_{k} w-\frac{1}{\left(1+\kappa_{Y}\right)^{2}} \partial_{1, \mu}^{2} \partial_{k} w=\partial_{k} h_{1}-\left(\partial_{k} \frac{1}{\mu}\right) w+\frac{1}{\left(1+\kappa_{Y}\right)^{2}} \partial_{1}\left(\partial_{k} \frac{1}{\mu^{3}}\right) \partial_{1} w=: \chi\left(h_{1}\right)
$$

in $\mathcal{D}\left(\partial_{1}\right)^{*}$. The function $w$ is next approximated by regularized functions. ${ }^{1}$ The mappings $h_{1}$ and $\mu$ are first extended to functions $\tilde{h}_{1} \in \mathrm{H}^{1}\left(\mathbb{R}^{3}\right)$ and $\tilde{\mu} \in \mathrm{W}^{1, \infty}\left(\mathbb{R}^{3}\right)$ by means of Stein's extension operator. The extensions are then restricted to $\left[a_{1}^{-}, a_{1}^{+}\right] \times \mathbb{R}^{2}=: \check{Q}$. We further generalize $L_{1}$ to functions on $\check{Q}$ by defining the operator

$$
\tilde{L}_{1} g:=\frac{1}{\tilde{\mu}} g-\frac{1}{\left(1+\kappa_{Y}\right)^{2}} \partial_{1} \frac{1}{\tilde{\mu}^{3}} \partial_{1} g, \quad g \in \mathcal{D}\left(\tilde{L}_{1}\right):=\left\{u \in \mathrm{L}^{2}(\check{Q}) \mid \partial_{1} u, \partial_{1}^{2} u \in \mathrm{L}^{2}(\check{Q}), u=0 \text { on }\left\{a_{1}^{ \pm}\right\} \times \mathbb{R}^{2}\right\} .
$$

The Lax-Milgram Lemma now yields a unique map $\tilde{w} \in \mathcal{D}\left(\tilde{L}_{1}\right)$ with $\tilde{L}_{1} \tilde{w}=\tilde{h}_{1}$. By uniqueness, $\left.\tilde{w}\right|_{Q}$ coincides with $w$. To transfer the arguments leading to (3.23) also to $\check{Q}$, we consider the domains

$$
\begin{aligned}
& \mathcal{D}\left(\tilde{\partial}_{1}\right):=\left\{u \in \mathrm{L}^{2}(\check{Q}) \mid \partial_{1} u \in \mathrm{L}^{2}(\check{Q}), u=0 \text { on }\left\{a_{1}^{ \pm}\right\} \times \mathbb{R}^{2}\right\}, \\
& \mathcal{D}\left(\tilde{\partial}_{k}\right):=\left\{u \in \mathrm{L}^{2}(\check{Q}) \mid \partial_{k} u \in \mathrm{L}^{2}(\check{Q})\right\}, \quad k \in\{2,3\} .
\end{aligned}
$$

for the partial derivative operators $\partial_{1}$ and $\partial_{k}$. We then derive as in (3.23) the formula

$$
\frac{1}{\tilde{\mu}} \partial_{k} \tilde{w}-\frac{1}{\left(1+\kappa_{Y}\right)^{2}} \partial_{1} \frac{1}{\tilde{\mu}^{3}} \partial_{1} \partial_{k} \tilde{w}=\partial_{k} \tilde{h}_{1}-\left(\partial_{k} \frac{1}{\tilde{\mu}}\right) \tilde{w}+\frac{1}{\left(1+\kappa_{Y}\right)^{2}} \partial_{1}\left(\partial_{k} \frac{1}{\tilde{\mu}^{3}}\right) \partial_{1} \tilde{w}=: \chi\left(\tilde{h}_{1}\right)
$$

in $\mathcal{D}\left(\tilde{\partial}_{1}\right)^{*}$.

The next step is to mollify $\tilde{w}$. Let $\rho_{n}^{k}: \mathbb{R} \rightarrow[0,1]$ be the smooth standard mollifier with support in $\left[-\frac{1}{n}, \frac{1}{n}\right]$ that acts on $x_{k}$, and denote the corresponding convolution operator by $M_{n}^{k}$ for $n \in \mathbb{N}$, i.e., $M_{n}^{k} f:=\rho_{n}^{k} * f$ for $f \in \mathrm{L}^{2}(\check{Q})$. The convolution operator with respect to $\rho_{n}^{k}(-\cdot)$ is called $M_{-n}^{k}$. In view of the equation

$$
\left\langle M_{n}^{k} f, \varphi\right\rangle_{\mathcal{D}\left(\tilde{\partial}_{1}\right)^{*} \times \mathcal{D}\left(\tilde{\partial}_{1}\right)}=\left\langle f, M_{-n}^{k} \varphi\right\rangle_{\mathcal{D}\left(\tilde{\partial}_{1}\right)^{*} \times \mathcal{D}\left(\tilde{\partial}_{1}\right)}, \quad f \in \mathcal{D}\left(\tilde{\partial}_{1}\right),
$$

and the inclusion $M_{-n}^{k}\left(\mathcal{D}\left(\tilde{\partial}_{1}\right)\right) \subseteq \mathcal{D}\left(\tilde{\partial}_{1}\right)$, the operator $M_{n}^{k}$ can be extended in a continuous way to the space $\mathcal{D}\left(\tilde{\partial}_{1}\right)^{*}$ via

$$
\left\langle M_{n}^{k} f, \varphi\right\rangle_{\mathcal{D}\left(\tilde{\partial}_{1}\right)^{*} \times \mathcal{D}\left(\tilde{\partial}_{1}\right)}:=\left\langle f, M_{-n}^{k} \varphi\right\rangle_{\mathcal{D}\left(\tilde{\partial}_{1}\right)^{*} \times \mathcal{D}\left(\tilde{\partial}_{1}\right)}, \quad f \in \mathcal{D}\left(\tilde{\partial}_{1}\right)^{*}, \varphi \in \mathcal{D}\left(\tilde{\partial}_{1}\right)
$$

\footnotetext{
${ }^{1}$ We here close a gap in the proof of Lemma 3.5 in [9].
} 
with $\left\|M_{n}^{k} f\right\|_{\mathcal{D}\left(\tilde{\partial}_{1}\right)^{*}} \leq\|f\|_{\mathcal{D}\left(\tilde{\partial}_{1}\right)^{*}}$ for $f \in \mathcal{D}\left(\tilde{\partial}_{1}\right)^{*}$, compare the proof of Lemma 4.1 in [29]. Standard mollifier theory further yields the convergence result

$$
\left|\left\langle M_{n}^{k} f-f, \varphi\right\rangle_{\mathcal{D}\left(\tilde{\partial}_{1}\right)^{*} \times \mathcal{D}\left(\tilde{\partial}_{1}\right)}\right|=\left|\left\langle f, M_{-n}^{k} \varphi-\varphi\right\rangle_{\mathcal{D}\left(\tilde{\partial}_{1}\right)^{*} \times \mathcal{D}\left(\tilde{\partial}_{1}\right)}\right| \rightarrow 0, \quad n \rightarrow \infty,
$$

and thus $M_{n}^{k} f \rightarrow f$ weakly* in $\mathcal{D}\left(\tilde{\partial}_{1}\right)^{*}$ for $f \in \mathcal{D}\left(\tilde{\partial}_{1}\right)^{*}$. Define now $\tilde{w}_{n}:=M_{n}^{k} \tilde{w}$ for $n \in \mathbb{N}$. The functions $\tilde{w}_{n}$ and $\partial_{k} \tilde{w}_{n}$ then belong to $\mathcal{D}\left(\tilde{L}_{1}\right)$ by construction. Note further that $\tilde{w}_{n} \rightarrow \tilde{w}$ in $\mathrm{L}^{2}(\check{Q})$ by classical mollifier theory.

We will next show that $\left(\partial_{k} \tilde{w}_{n}\right)_{n}$ has a weak limit in $\mathcal{D}\left(\tilde{\partial}_{1}\right)$. A computation first reveals the identities

$$
\begin{aligned}
\tilde{L}_{1} \partial_{k} \tilde{w}_{n}= & \frac{1}{\tilde{\mu}} \partial_{k} M_{n}^{k} \tilde{w}-\frac{1}{\left(1+\kappa_{Y}\right)^{2}} \partial_{1} \frac{1}{\tilde{\mu}^{3}} \partial_{k} M_{n}^{k} \partial_{1} \tilde{w} \\
= & \left(\frac{1}{\tilde{\mu}} \partial_{k} M_{n}^{k} \tilde{w}-M_{n}^{k}\left(\frac{1}{\tilde{\mu}} \partial_{k} \tilde{w}\right)\right)+M_{n}^{k}\left(\frac{1}{\tilde{\mu}} \partial_{k} \tilde{w}-\frac{1}{\left(1+\kappa_{Y}\right)^{2}} \partial_{1} \frac{1}{\tilde{\mu}^{3}} \partial_{1} \partial_{k} \tilde{w}\right) \\
& +\frac{1}{\left(1+\kappa_{Y}\right)^{2}} \partial_{1}\left(M_{n}^{k}\left(\frac{1}{\tilde{\mu}^{3}} \partial_{k} \partial_{1} \tilde{w}\right)-\frac{1}{\tilde{\mu}^{3}} \partial_{k} M_{n}^{k}\left(\partial_{1} \tilde{w}\right)\right) \\
= & e_{1, n}+e_{2, n}+e_{3, n} .
\end{aligned}
$$

The summands $e_{1, n}$ and $e_{3, n}$ converge to zero in $\mathcal{D}\left(\tilde{\partial}_{1}\right)^{*}$ as $n \rightarrow \infty$ by Theorem C.14 in [4]. In consideration of (3.24) and (3.25), the second summand $e_{2, n}$ tends weakly* in $\mathcal{D}\left(\tilde{\partial}_{1}\right)^{*}$ to $\chi\left(\tilde{h}_{1}\right)$. In the following we extrapolate $\tilde{L}_{1}$. Since $\tilde{L}_{1}$ is the associated operator to the bilinear form

$$
\mathcal{D}\left(\tilde{\partial}_{1}\right)^{2} \rightarrow \mathbb{R}, \quad\left(w_{1}, w_{2}\right) \mapsto\left(\frac{1}{\tilde{\mu}} w_{1}, w_{2}\right)_{\mathrm{L}^{2}(\check{Q})}+\frac{1}{\left(1+\kappa_{Y}\right)^{2}}\left(\frac{1}{\tilde{\mu}^{3}} \partial_{1} w_{1}, \partial_{1} w_{2}\right)_{\mathrm{L}^{2}(\check{Q})},
$$

which is closed, symmetric, positive definite and densely defined on $\mathrm{L}^{2}(\check{Q})^{2}$, Theorem VI.2.7 in [21] shows that $\tilde{L}_{1}$ is selfadjoint on $\mathrm{L}^{2}(\check{Q})$. Theorem VI.2.23 in $[21]$ further yields the identity $\mathcal{D}\left(\tilde{\partial}_{1}\right)=\mathcal{D}\left(\tilde{L}_{1}^{1 / 2}\right)$. Denote by $\mathrm{L}^{2}(\check{Q})_{q}$ for $q \in \mathbb{Q} \backslash\{0\}$ the fractional extrapolation space with respect to $\tilde{L}_{1}$, and let $\left(\tilde{L}_{1}\right)_{-1}^{-1}$ be the bounded inverse of the extrapolation operator $\left(\tilde{L}_{1}\right)_{-1}: \mathrm{L}^{2}(\check{Q}) \rightarrow \mathrm{L}^{2}(\check{Q})_{-1}$. Employing the isomorphies

$$
\mathcal{D}\left(\tilde{\partial}_{1}\right)^{*}=\mathcal{D}\left(\tilde{L}_{1}^{1 / 2}\right)^{*} \cong \mathrm{L}^{2}(\check{Q})_{-1 / 2}
$$

see Theorem 1.4.12 in [2], we infer that $\left(\tilde{L}_{1}\right)_{-1}^{-1}: \mathcal{D}\left(\tilde{\partial}_{1}\right)^{*} \rightarrow \mathcal{D}\left(\tilde{\partial}_{1}\right)$ is bounded. The functions $\partial_{k} \tilde{w}_{n}$ hence converge weakly in $\mathcal{D}\left(\tilde{\partial}_{1}\right)$ to $\left(\tilde{L}_{1}\right)_{-1}^{-1}\left(\chi\left(\tilde{h}_{1}\right)\right)=: v$ as $n \rightarrow \infty$. This in particular implies weak convergence in $\mathcal{D}\left(\tilde{\partial}_{k}\right)^{*}$. By definition of $\tilde{w}_{n}$, however, $\left(\partial_{k} \tilde{w}_{n}\right)_{n}$ also has the weak limit $\partial_{k} \tilde{w}$ in $\mathcal{D}\left(\tilde{\partial}_{k}\right)^{*}$. By uniqueness, $\partial_{k} \tilde{w}$ coincides with $v$ and belongs to $\mathcal{D}\left(\tilde{\partial}_{1}\right)$. $\Gamma_{1}$

Recalling the choice $\mathbf{H}_{1}=\frac{1}{\mu} w=\left.\left(\frac{1}{\tilde{\mu}} \tilde{w}\right)\right|_{Q}$, both functions $\mathbf{H}_{1}$ and $\partial_{1} \mathbf{H}_{1}$ are contained in $\mathrm{H}^{1}(Q)$, and $\mathbf{H}_{1}=0$ on

3) The results of Step 2) show that $\Phi=\frac{1}{1+\kappa_{Y}}\left(\check{\Phi}-\frac{1}{\mu^{2}} \partial_{1}\left(\mu \mathbf{H}_{1}\right)\right)$ belongs to $\mathrm{H}^{1}(Q)$, and system (3.20) leads to the relations

$$
\partial_{1} \frac{1}{\mu} \Phi=\frac{1}{1+\kappa_{Y}}\left(\partial_{1} \frac{1}{\mu} \check{\Phi}-\partial_{1} \frac{1}{\mu^{3}} \partial_{1} \mu \mathbf{H}_{1}\right)=\check{\mathbf{H}}_{1}-\left(1+\kappa_{Y}\right) \mathbf{H}_{1} \in \mathrm{H}^{1}(Q), \quad \partial_{1} \frac{1}{\mu} \Phi=0 \quad \text { on } \Gamma_{1} .
$$

Altogether, $(\mathbf{E}, \mathbf{H}, \Phi)$ is an element of $\mathcal{D}\left(D_{1, Y}\right)$ and satisfies $\left(\left(1+\kappa_{Y}\right) I-D_{1, Y}\right)(\mathbf{E}, \mathbf{H}, \Phi)=(\check{\mathbf{E}}, \check{\mathbf{H}}, \check{\Phi})$.

The succeeding corollary is a conclusion of Lemmas 3.12-3.14, and is crucial for the stability of the schemes (3.17) and (3.18) in $Y$.

Corollary 3.15. Let $i \in\{1,2,3\}$. The resolvent $\left(I \pm \tau D_{i, Y}\right)^{-1}: Y \rightarrow \mathcal{D}\left(D_{i, Y}\right)$ exists, coincides with the restriction of $\left(I \pm \tau D_{i}\right)^{-1}$ to $Y$, and is bounded by

$$
\left\|\left(I \pm \tau D_{i, Y}\right)^{-1}\right\|_{\mathscr{B}(Y)} \leq \frac{1}{1-\tau \kappa_{Y}}
$$

for $\tau \in\left(0, \frac{1}{\kappa_{Y}}\right)$. There further is a constant $\tau_{0} \in\left(0, \frac{1}{2 \kappa_{Y}}\right)$ with

$$
\left\|S_{\tau}\left(D_{i, Y}\right)\right\|_{\mathscr{B}(Y)} \leq \mathrm{e}^{3 \kappa_{Y} \tau}
$$

for $\tau \in\left(0, \tau_{0}\right)$. 
Proof. Lemmas 3.12-3.14 at hand, the same arguments as in the proof for Proposition 3.6 in [9] imply the asserted statements.

We can choose the same constant $\tau_{0}$ in Lemma 3.11 and Corollary 3.15. To conclude that the iterates of the damped scheme (3.18) stay in $Y$ if the starting value is chosen within $Y$, it remains to show that the damping operators from (3.16) restrict to operators on $Y$.

Lemma 3.16. Let $\tau \in\left(0, \min \left\{1, \frac{\sqrt{2}}{\kappa_{Y}}\right\}\right)$ and $L \in\left\{A, B, D_{1}, D_{2}, D_{3}\right\}$. The operator $V_{\tau}(L)$ leaves $Y$ invariant.

Proof. It suffices to consider the case $L=A$ since all others can be treated in a similar fashion. Employing the identities

$$
\begin{aligned}
V_{\tau}(A) & =\left(I-\frac{\tau^{3}}{4} A^{2}\left(I-\frac{\tau^{2}}{4} A^{2}\right)^{-1}\right)^{-1}=\left(I-\frac{\tau^{2}}{4} A^{2}\right)\left(I-\frac{\tau^{2}+\tau^{3}}{4} A^{2}\right)^{-1} \\
& =\left(I+\frac{\tau}{2} A\right)\left(I+\frac{\sqrt{\tau^{2}+\tau^{3}}}{2} A\right)^{-1}\left(I-\frac{\tau}{2} A\right)\left(I-\frac{\sqrt{\tau^{2}+\tau^{3}}}{2} A\right)^{-1}
\end{aligned}
$$

on $X$ and Lemma 3.11, the inclusion $V_{\tau}(A)(Y) \subseteq Y$ directly follows.

Besides its uniform exponential stability and convergence with order 1, see Theorems 3.9 and 6.5, the proposed ADI scheme (3.18) has only linear complexity. The next remark deals with this issue.

Remark 3.17. We here deduce that essentially only one-dimensional elliptic problems have to be solved implicitly in each iteration of (3.18). Formula (3.27) already leads to the representation

$$
\begin{aligned}
\left(\begin{array}{l}
\mathbf{E}^{n+1} \\
\mathbf{H}^{n+1} \\
\Phi^{n+1}
\end{array}\right)= & \left(\begin{array}{ccc}
\mathrm{e}^{-\tau \sigma} & 0 & 0 \\
0 & I & 0 \\
0 & 0 & \mathrm{e}^{-\tau \eta}
\end{array}\right) \prod_{i=1}^{3}\left(\left(I+\frac{\tau}{2} D_{i}\right)^{2}\left(I+\frac{\sqrt{\tau^{2}+\tau^{3}}}{2} D_{i}\right)^{-1}\left(I-\frac{\sqrt{\tau^{2}+\tau^{3}}}{2} D_{i}\right)^{-1}\right)\left(I+\frac{\tau}{2} B\right)^{2} \\
& \cdot\left(I+\frac{\sqrt{\tau^{2}+\tau^{3}}}{2} B\right)^{-1}\left(I-\frac{\sqrt{\tau^{2}+\tau^{3}}}{2} B\right)^{-1}\left(I+\frac{\tau}{2} A\right)^{2}\left(I+\frac{\sqrt{\tau^{2}+\tau^{3}}}{2} A\right)^{-1}\left(I-\frac{\sqrt{\tau^{2}+\tau^{3}}}{2} A\right)^{-1}\left(\begin{array}{c}
\mathbf{E}^{n} \\
\mathbf{H}^{n} \\
\Phi^{n}
\end{array}\right), \quad n \in \mathbb{N}
\end{aligned}
$$

of scheme (3.18). The main effort in the evaluation of (3.18) consequently consists in the implicit steps due to the involved resolvent operators. Since it is well known that the application of the resolvents of $A$ and $B$ leads to essentially one-dimensional problems, see $[24,33,18,9]$, we deal only with the operator $\left(I+\lambda D_{1}\right)^{-1}$ for $\lambda \in\left(-\frac{1}{\kappa_{Y}}, \frac{1}{\kappa_{Y}}\right)$.

Similar to [9], we restrict ourselves to the case of initial data $\left(\mathbf{E}^{0}, \mathbf{H}^{0}, \Phi^{0}\right) \in Y$. Lemmas 3.11 and 3.16 , and Corollary 3.15 then show that all iterates of (3.18) remain in $Y$. In particular, the resolvent operator is applied to a vector $(\mathbf{E}, \mathbf{H}, \Phi) \in Y$. Let $(\tilde{\mathbf{E}}, \tilde{\mathbf{H}}, \tilde{\Phi}):=\left(I+\lambda D_{1}\right)^{-1}(\mathbf{E}, \mathbf{H}, \Phi) \in \mathcal{D}\left(D_{1, Y}\right)$. The definition of $D_{1}$ then leads to the system

$$
\begin{aligned}
\tilde{\mathbf{E}} & =\mathbf{E}, \\
\tilde{\mathbf{H}}_{j} & =\mathbf{H}_{j} \quad \text { for } j \in\{2,3\}, \\
\tilde{\mathbf{H}}_{1}-\lambda \partial_{1}\left(\frac{1}{\mu} \tilde{\Phi}\right) & =\mathbf{H}_{1}, \\
\tilde{\Phi}-\frac{\lambda}{\mu^{2}} \partial_{1}\left(\mu \tilde{\mathbf{H}}_{1}\right) & =\Phi .
\end{aligned}
$$

In view of the above regularity considerations, the last identity can be inserted into the third to obtain the formula

$$
\tilde{\mathbf{H}}_{1}-\lambda^{2} \partial_{1}\left(\frac{1}{\mu^{3}} \partial_{1} \mu \tilde{\mathbf{H}}_{1}\right)=\mathbf{H}_{1}+\lambda \partial_{1}\left(\frac{1}{\mu} \Phi\right) .
$$

As a result, a sole one-dimensional elliptic problem has to be solved for the evaluation of $\left(I+\lambda D_{1}\right)^{-1}$, and all other calculations can be done explicit. 


\section{A UNIFORM OBSERVABILITY INEQUALITY}

This section is devoted to the derivation of an internal observability estimate for the conserving scheme (3.17). Ideas from [26] for the continuous setting will be employed, and the most important ingredient of our arguments is a discrete version of the multiplier method. Before we state the observability inequality, however, we divide the scheme (3.17) into appropriate substeps and derive useful difference equations.

\subsection{Difference equations for the conserving scheme}

Let $n \in \mathbb{N}_{0}, i \in\{1,2,3\}$, and $\tau \in\left(0, \min \left\{\frac{1}{2}, \frac{\sqrt{2}}{\kappa_{Y}}\right\}\right)$. We divide the scheme (3.17) into the substeps

$$
\begin{aligned}
& \left(\begin{array}{l}
\mathbf{E}_{c}^{n, 1} \\
\mathbf{H}_{c}^{n, 1} \\
\Phi_{c}^{n, 1}
\end{array}\right):=\left(I-\frac{\tau}{2} A\right)^{-1}\left(\begin{array}{l}
\mathbf{E}_{c}^{n} \\
\mathbf{H}_{c}^{n} \\
\Phi_{c}^{n}
\end{array}\right) ;\left(\begin{array}{l}
\mathbf{E}_{c}^{n, 2} \\
\mathbf{H}_{c}^{n, 2} \\
\Phi_{c}^{n, 2}
\end{array}\right):=\left(I+\frac{\tau}{2} A\right)\left(\begin{array}{l}
\mathbf{E}_{c}^{n, 1} \\
\mathbf{H}_{c}^{n, 1} \\
\Phi_{c}^{n, 1}
\end{array}\right) ;\left(\begin{array}{l}
\mathbf{E}_{c}^{n, 3} \\
\mathbf{H}_{c}^{n, 3} \\
\Phi_{c}^{n, 3}
\end{array}\right):=\left(I-\frac{\tau}{2} B\right)^{-1}\left(\begin{array}{c}
\mathbf{E}_{c}^{n, 2} \\
\mathbf{H}_{c}^{n, 2} \\
\Phi_{c}^{n, 2}
\end{array}\right) ; \\
& \left(\begin{array}{l}
\mathbf{E}_{c}^{n, 4} \\
\mathbf{H}_{c}^{n, 4} \\
\Phi_{c}^{n, 4}
\end{array}\right):=\left(I+\frac{\tau}{2} B\right)\left(\begin{array}{c}
\mathbf{E}_{c}^{n, 3} \\
\mathbf{H}_{c}^{n, 3} \\
\Phi_{c}^{n, 3}
\end{array}\right) ;\left(\begin{array}{l}
\mathbf{E}_{c}^{n, 3+2 i} \\
\mathbf{H}_{c}^{n, 3+2 i} \\
\Phi_{c}^{n, 3+2 i}
\end{array}\right):=\left(I-\frac{\tau}{2} D_{i}\right)^{-1}\left(\begin{array}{c}
\mathbf{E}_{c}^{n, 2+2 i} \\
\mathbf{H}_{c}^{n, 2+2 i} \\
\Phi_{c}^{n, 2+2 i}
\end{array}\right) ;\left(\begin{array}{l}
\mathbf{E}_{c}^{n, 4+2 i} \\
\mathbf{H}_{c}^{n, 4+2 i} \\
\Phi_{c}^{n, 4+2 i}
\end{array}\right):=\left(I+\frac{\tau}{2} D_{i}\right)\left(\begin{array}{l}
\mathbf{E}_{c}^{n, 3+2 i} \\
\mathbf{H}_{c}^{n, 3+2 i} \\
\Phi_{c}^{n, 3+2 i}
\end{array}\right)
\end{aligned}
$$

to derive crucial relations between the succeeding iterates $\left(\mathbf{E}_{c}^{n}, \mathbf{H}_{c}^{n}, \Phi_{c}^{n}\right)$ and $\left(\mathbf{E}_{c}^{n+1}, \mathbf{H}_{c}^{n+1}, \Phi_{c}^{n+1}\right)$. From now on we assume in this section that the initial data $\left(\mathbf{E}_{c}^{0}, \mathbf{H}_{c}^{0}, \Phi_{c}^{0}\right)$ belongs to $Y$, and the next remark recalls the resulting consequences of this assumption.

Remark 4.1. Lemma 3.11 and Corollary 3.15 imply that all intermediate steps and the next iterate of (3.17) remain in $Y$. The substeps with odd index are even contained in the respective domain $\mathcal{D}\left(A_{Y}\right), \mathcal{D}\left(B_{Y}\right)$, or $\mathcal{D}\left(D_{i, Y}\right)$ for $i \in\{1,2,3\}$, respectively.

The remark is crucial for the proof of the observability inequality for (3.17), since it enables integration by parts with vanishing boundary integrals, roughly speaking. The stated regularity facts also justify the following calculations in $\mathrm{L}^{2}$.

Denoting the $l$-th component of the vector $\mathbf{H}_{c}^{n, 3+2 i}$ by $\mathbf{H}_{c, l}^{n, 3+2 i}$ and the $l$-th standard unit vector by $\mathbf{e}_{l}$, the identities

$$
\begin{array}{rlrl}
\left(\begin{array}{c}
\mathbf{E}_{c}^{n, 2}+\mathbf{E}_{c}^{n} \\
\mathbf{H}_{c}^{n, 2}+\mathbf{H}_{c}^{n} \\
\Phi_{c}^{n, 2}+\Phi_{c}^{n}
\end{array}\right) & =2\left(\begin{array}{c}
\mathbf{E}_{c}^{n, 1} \\
\mathbf{H}_{c}^{n, 1} \\
\Phi_{c}^{n, 1}
\end{array}\right), & \left(\begin{array}{c}
\mathbf{E}_{c}^{n, 2}-\mathbf{E}_{c}^{n} \\
\mathbf{H}_{c}^{n, 2}-\mathbf{H}_{c}^{n} \\
\Phi_{c}^{n, 2}-\Phi_{c}^{n}
\end{array}\right)=\tau\left(\begin{array}{c}
\frac{1}{\varepsilon} \mathscr{C}_{1} \mathbf{H}_{c}^{n, 1} \\
\frac{1}{\mu} \mathscr{C}_{2} \mathbf{E}_{c}^{n, 1} \\
0
\end{array}\right), \\
\left(\begin{array}{c}
\mathbf{E}_{c}^{n, 4}+\mathbf{E}_{c}^{n, 2} \\
\mathbf{H}_{c}^{n, 4}+\mathbf{H}_{c}^{n, 2} \\
\Phi_{c}^{n, 4}+\Phi_{c}^{n, 2}
\end{array}\right) & =2\left(\begin{array}{c}
\mathbf{E}_{c}^{n, 3} \\
\mathbf{H}_{c}^{n, 3} \\
\Phi_{c}^{n, 3}
\end{array}\right), & \left(\begin{array}{c}
\mathbf{E}_{c}^{n, 4}-\mathbf{E}_{c}^{n, 2} \\
\mathbf{H}_{c}^{n, 4}-\mathbf{H}_{c}^{n, 2} \\
\Phi_{c}^{n, 4}-\Phi_{c}^{n, 2}
\end{array}\right) & =-\tau\left(\begin{array}{c}
\frac{1}{\varepsilon} \mathscr{C}_{2} \mathbf{H}_{c}^{n, 3} \\
\frac{1}{\mu} \mathscr{C}_{1} \mathbf{E}_{c}^{n, 3} \\
0
\end{array}\right), \\
\left(\begin{array}{c}
\mathbf{E}_{c}^{n, 4+2 i}+\mathbf{E}_{c}^{n, 2+2 i} \\
\mathbf{H}_{c}^{n, 4+2 i}+\mathbf{H}_{c}^{n, 2+2 i} \\
\Phi_{c}^{n, 4+2 i}+\Phi_{c}^{n, 2+2 i}
\end{array}\right) & =2\left(\begin{array}{c}
\mathbf{E}_{c}^{n, 3+2 i} \\
\mathbf{H}_{c}^{n, 3+2 i} \\
\Phi_{c}^{n, 3+2 i}
\end{array}\right), & \left(\begin{array}{c}
\mathbf{E}_{c}^{n, 4+2 i}-\mathbf{E}_{c}^{n, 2+2 i} \\
\mathbf{H}_{c}^{n, 4+2 i}-\mathbf{H}_{c}^{n, 2+2 i} \\
\Phi_{c}^{n, 4+2 i}-\Phi_{c}^{n, 2+2 i}
\end{array}\right) & =-\tau\left(\begin{array}{c}
\partial_{i}\left(\frac{1}{\mu} \Phi_{c}^{n, 3+2 i}\right) \mathbf{e}_{i} \\
\frac{1}{\mu^{2}} \partial_{i}\left(\mu \mathbf{H}_{c, i}^{n, 3+2 i}\right)
\end{array}\right)
\end{array}
$$

immediately follow from (4.1). The relations (4.1)-(4.4) and $\mathbf{E}_{c}^{n+1}=\mathbf{E}_{c}^{n, 4}$ then lead to the difference equations

$$
\begin{aligned}
\frac{1}{\tau}\left(\mathbf{E}_{c}^{n+1}-\mathbf{E}_{c}^{n}\right) & =\frac{1}{\tau}\left(\mathbf{E}_{c}^{n, 4}-\mathbf{E}_{c}^{n, 2}\right)+\frac{1}{\tau}\left(\mathbf{E}_{c}^{n, 2}-\mathbf{E}_{c}^{n}\right)=-\frac{1}{\varepsilon} \mathscr{C}_{2} \mathbf{H}_{c}^{n, 3}+\frac{1}{\varepsilon} \mathscr{C}_{1} \mathbf{H}_{c}^{n, 1} \\
& =-\frac{1}{\varepsilon} \mathscr{C}_{2} \mathbf{H}_{c}^{n, 2}+\frac{\tau}{2 \varepsilon} \mathscr{C}_{2} \frac{1}{\mu} \mathscr{C}_{1} \mathbf{E}_{c}^{n, 3}+\frac{1}{\varepsilon} \mathscr{C}_{1} \mathbf{H}_{c}^{n, 2}-\frac{\tau}{2 \varepsilon} \mathscr{C}_{1} \frac{1}{\mu} \mathscr{C}_{2} \mathbf{E}_{c}^{n, 1} \\
\frac{1}{\tau}\left(\mathbf{H}_{c}^{n+1}-\mathbf{H}_{c}^{n}\right) & =\frac{1}{\tau}\left(\mathbf{H}_{c}^{n+1}-\mathbf{H}_{c}^{n, 4}\right)+\frac{1}{\tau}\left(\mathbf{H}_{c}^{n, 4}-\mathbf{H}_{c}^{n, 2}\right)+\frac{1}{\tau}\left(\mathbf{H}_{c}^{n, 2}-\mathbf{H}_{c}^{n}\right) \\
& =-\left(\begin{array}{c}
\partial_{1} \frac{1}{\mu} \Phi_{c}^{n, 5} \\
\partial_{2} \frac{1}{\mu} \Phi_{c}^{n, 7} \\
\partial_{3} \frac{1}{\mu} \Phi_{c}^{n, 9}
\end{array}\right)-\frac{1}{\mu} \mathscr{C}_{1} \mathbf{E}_{c}^{n, 3}+\frac{1}{\mu} \mathscr{C}_{2} \mathbf{E}_{c}^{n, 1}
\end{aligned}
$$




$$
=-\left(\begin{array}{c}
\partial_{1} \frac{1}{\mu} \Phi_{c}^{n, 5} \\
\partial_{2} \frac{1}{\mu} \Phi_{c}^{n, 7} \\
\partial_{3} \frac{1}{\mu} \Phi_{c}^{n, 9}
\end{array}\right)-\frac{1}{\mu} \mathscr{C}_{1} \mathbf{E}_{c}^{n, 2}+\frac{\tau}{2 \mu} \mathscr{C}_{1} \frac{1}{\varepsilon} \mathscr{C}_{2} \mathbf{H}_{c}^{n, 3}+\frac{1}{\mu} \mathscr{C}_{2} \mathbf{E}_{c}^{n, 2}-\frac{\tau}{2 \mu} \mathscr{C}_{2} \frac{1}{\varepsilon} \mathscr{C}_{1} \mathbf{H}_{c}^{n, 1} .
$$

Another consequence of (4.1)-(4.4) and $\mathbf{E}_{c}^{n+1}=\mathbf{E}_{c}^{n, 4}$ are the identities

$$
\begin{aligned}
\frac{1}{2}\left(\mathbf{E}_{c}^{n+1}+\mathbf{E}_{c}^{n}\right) & =\frac{1}{2}\left(\mathbf{E}_{c}^{n, 4}+\mathbf{E}_{c}^{n, 2}\right)-\frac{1}{2}\left(\mathbf{E}_{c}^{n, 2}-\mathbf{E}_{c}^{n}\right)=\mathbf{E}_{c}^{n, 2}-\frac{\tau}{2 \varepsilon} \mathscr{C}_{2} \mathbf{H}_{c}^{n, 3}-\frac{\tau}{2 \varepsilon} \mathscr{C}_{1} \mathbf{H}_{c}^{n, 1}, \\
\frac{1}{2}\left(\mathbf{H}_{c}^{n+1}+\mathbf{H}_{c}^{n}\right) & =\frac{1}{2}\left(\mathbf{H}_{c}^{n+1}-\mathbf{H}_{c}^{n, 4}\right)+\frac{1}{2}\left(\mathbf{H}_{c}^{n, 4}+\mathbf{H}_{c}^{n, 2}\right)-\frac{1}{2}\left(\mathbf{H}_{c}^{n, 2}-\mathbf{H}_{c}^{n}\right) \\
& =-\frac{\tau}{2}\left(\begin{array}{c}
\partial_{1} \frac{1}{\mu} \Phi_{c}^{n, 5} \\
\partial_{2} \frac{1}{\mu} \Phi_{c}^{n, 7} \\
\partial_{3} \frac{1}{\mu} \Phi_{c}^{n, 9}
\end{array}\right)+\mathbf{H}_{c}^{n, 2}-\frac{\tau}{2 \mu} \mathscr{C}_{1} \mathbf{E}_{c}^{n, 3}-\frac{\tau}{2 \mu} \mathscr{C}_{2} \mathbf{E}_{c}^{n, 1} .
\end{aligned}
$$

Inserting these formulas into (4.5) and (4.7), and using the splitting relation curl $=\mathscr{C}_{1}-\mathscr{C}_{2}$, we have thus derived the fundamental difference equations

$$
\begin{aligned}
& \frac{1}{\tau}\left(\mathbf{E}_{c}^{n+1}-\mathbf{E}_{c}^{n}\right)=\frac{1}{2 \varepsilon} \operatorname{curl}\left(\mathbf{H}_{c}^{n+1}+\mathbf{H}_{c}^{n}\right)+\frac{\tau}{2 \varepsilon} \operatorname{curl}\left(\begin{array}{c}
\partial_{1} \frac{1}{\mu} \Phi_{c}^{n, 5} \\
\partial_{2} \frac{1}{\mu} \Phi_{c}^{n, 7} \\
\partial_{3} \frac{1}{\mu} \Phi_{c}^{n, 9}
\end{array}\right)-\frac{\tau}{2 \varepsilon} \mathscr{C}_{2} \frac{1}{\mu} \mathscr{C}_{2} \mathbf{E}_{c}^{n, 1}+\frac{\tau}{2 \varepsilon} \mathscr{C}_{1} \frac{1}{\mu} \mathscr{C}_{1} \mathbf{E}_{c}^{n, 3}, \\
& \frac{1}{\tau}\left(\mathbf{H}_{c}^{n+1}-\mathbf{H}_{c}^{n}\right)=-\frac{1}{2 \mu} \operatorname{curl}\left(\mathbf{E}_{c}^{n+1}+\mathbf{E}_{c}^{n}\right)+\frac{\tau}{2 \mu} \mathscr{C}_{2} \frac{1}{\varepsilon} \mathscr{C}_{2} \mathbf{H}_{c}^{n, 3}-\frac{\tau}{2 \mu} \mathscr{C}_{1} \frac{1}{\varepsilon} \mathscr{C}_{1} \mathbf{H}_{c}^{n, 1}-\left(\begin{array}{c}
\partial_{1} \frac{1}{\mu} \Phi_{c}^{n, 5} \\
\partial_{2} \frac{1}{\mu} \Phi_{c}^{n, 7} \\
\partial_{3} \frac{1}{\mu} \Phi_{c}^{n, 9}
\end{array}\right) .
\end{aligned}
$$

These identities correspond to a perturbed discrete version of the homogeneous extended Maxwell equations. In view of (4.10), it is useful to have yet another representation of the last expression on the right hand side. From (4.4) the auxiliary relations

$$
\begin{aligned}
& 2 \Phi_{c}^{n, 5}=\Phi_{c}^{n, 6}+\Phi_{c}^{n, 4}=2 \Phi_{c}^{n, 6}+\frac{\tau}{\mu^{2}} \partial_{1} \mu \mathbf{H}_{c, 1}^{n, 5}, \\
& 2 \Phi_{c}^{n, 7}=\Phi_{c}^{n, 8}+\Phi_{c}^{n, 6}=2 \Phi_{c}^{n, 6}-\frac{\tau}{\mu^{2}} \partial_{2} \mu \mathbf{H}_{c, 2}^{n, 7}, \\
& 2 \Phi_{c}^{n, 9}=\Phi_{c}^{n+1}+\Phi_{c}^{n, 8}=2 \Phi_{c}^{n, 8}-\frac{\tau}{\mu^{2}} \partial_{3} \mu \mathbf{H}_{c, 3}^{n, 9}=2 \Phi_{c}^{n, 6}-2 \frac{\tau}{\mu^{2}} \partial_{2} \mu \mathbf{H}_{c, 2}^{n, 7}-\frac{\tau}{\mu^{2}} \partial_{3} \mu \mathbf{H}_{c, 3}^{n, 9}
\end{aligned}
$$

follow, and we conclude the result

$$
\left(\begin{array}{c}
\partial_{1} \frac{1}{\mu} \Phi_{c}^{n, 5} \\
\partial_{2} \frac{1}{\mu} \Phi_{c}^{n, 7} \\
\partial_{3} \frac{1}{\mu} \Phi_{c}^{n, 9}
\end{array}\right)=\nabla\left(\frac{1}{\mu} \Phi_{c}^{n, 6}\right)+\frac{\tau}{2}\left(\begin{array}{c}
\partial_{1} \frac{1}{\mu^{3}} \partial_{1} \mu \mathbf{H}_{c, 1}^{n, 5} \\
-\partial_{2} \frac{1}{\mu^{3}} \partial_{2} \mu \mathbf{H}_{c, 2}^{n, 7} \\
-2 \partial_{3} \frac{1}{\mu^{3}} \partial_{2} \mu \mathbf{H}_{c, 2}^{n, 7}-\partial_{3} \frac{1}{\mu^{3}} \partial_{3} \mu \mathbf{H}_{c, 3}^{n, 9}
\end{array}\right) .
$$

The last formula means that the vector $\left(\partial_{1} \frac{1}{\mu} \Phi_{c}^{n, 5}, \partial_{2} \frac{1}{\mu} \Phi_{c}^{n, 7}, \partial_{3} \frac{1}{\mu} \Phi_{c}^{n, 9}\right)$ is the gradient of a function plus some higher order error term. This observation is important when dealing with orthogonality properties of certain Helmholtz decompositions.

It remains to deduce a difference equation for $\Phi_{c}^{n}$. We first note the identities $\mathbf{H}_{c, 1}^{n+1}=\mathbf{H}_{c, 1}^{n, 6}, \mathbf{H}_{c, 2}^{n, 6}=\mathbf{H}_{c, 2}^{n, 4}$, $\mathbf{H}_{c, 2}^{n+1}=\mathbf{H}_{c, 2}^{n, 8}, \mathbf{H}_{c, 3}^{n, 8}=\mathbf{H}_{c, 3}^{n, 4}$, and obtain from (4.2)-(4.4) the supplementary relations

$$
\begin{aligned}
2 \mathbf{H}_{c, 1}^{n, 5} & =\mathbf{H}_{c, 1}^{n, 6}+\mathbf{H}_{c, 1}^{n, 4}=\mathbf{H}_{c, 1}^{n+1}+\mathbf{H}_{c, 1}^{n}+\left(\mathbf{H}_{c, 1}^{n, 4}-\mathbf{H}_{c, 1}^{n, 2}\right)+\left(\mathbf{H}_{c, 1}^{n, 2}-\mathbf{H}_{c, 1}^{n}\right) \\
& =\mathbf{H}_{c, 1}^{n+1}+\mathbf{H}_{c, 1}^{n}-\frac{\tau}{\mu}\left(\mathscr{C}_{1} \mathbf{E}_{c}^{n, 3}\right)_{1}+\frac{\tau}{\mu}\left(\mathscr{C}_{2} \mathbf{E}_{c}^{n, 1}\right)_{1}, \\
2 \mathbf{H}_{c, 2}^{n, 7} & =\mathbf{H}_{c, 2}^{n+1}+\mathbf{H}_{c, 2}^{n}-\frac{\tau}{\mu}\left(\mathscr{C}_{1} \mathbf{E}_{c}^{n, 3}\right)_{2}+\frac{\tau}{\mu}\left(\mathscr{C}_{2} \mathbf{E}_{c}^{n, 1}\right)_{2}, \\
2 \mathbf{H}_{c, 3}^{n, 9} & =\mathbf{H}_{c, 3}^{n+1}+\mathbf{H}_{c, 3}^{n}-\frac{\tau}{\mu}\left(\mathscr{C}_{1} \mathbf{E}_{c}^{n, 3}\right)_{3}+\frac{\tau}{\mu}\left(\mathscr{C}_{2} \mathbf{E}_{c}^{n, 1}\right)_{3} .
\end{aligned}
$$

Together with the formulas on the right hand side of (4.2)-(4.4) and in particular the relation $\Phi_{c}^{n, 4}=\Phi_{c}^{n}$, we thus infer the remaining difference equation

$$
\begin{aligned}
\frac{1}{\tau}\left(\Phi_{c}^{n+1}-\Phi_{c}^{n}\right) & =-\frac{1}{\mu^{2}} \partial_{1} \mu \mathbf{H}_{c, 1}^{n, 5}-\frac{1}{\mu^{2}} \partial_{2} \mu \mathbf{H}_{c, 2}^{n, 7}-\frac{1}{\mu^{2}} \partial_{3} \mu \mathbf{H}_{c, 3}^{n, 9} \\
& =-\frac{1}{2 \mu^{2}} \operatorname{div}\left(\mu\left(\mathbf{H}_{c}^{n+1}+\mathbf{H}_{c}^{n}\right)\right)+\frac{\tau}{2 \mu^{2}} \operatorname{div} \mathscr{C}_{1} \mathbf{E}_{c}^{n, 3}-\frac{\tau}{2 \mu^{2}} \operatorname{div} \mathscr{C}_{2} \mathbf{E}_{c}^{n, 1}
\end{aligned}
$$


corresponding to a perturbed discrete version of the differential equation for $\Phi$ in (3.1). It is further equivalent to the crucial divergence identity

$$
\frac{1}{2 \mu^{2}} \operatorname{div}\left(\mu\left(\mathbf{H}_{c}^{n+1}+\mathbf{H}_{c}^{n}\right)\right)=-\frac{1}{\tau}\left(\Phi_{c}^{n+1}-\Phi_{c}^{n}\right)+\frac{\tau}{2 \mu^{2}} \operatorname{div} \mathscr{C}_{1} \mathbf{E}_{c}^{n, 3}-\frac{\tau}{2 \mu^{2}} \operatorname{div} \mathscr{C}_{2} \mathbf{E}_{c}^{n, 1}
$$

The substeps from (4.1) at hand, we can now state the uniform interior observability inequality.

Theorem 4.2. Let $\varepsilon, \mu$ satisfy (1.2), $\stackrel{\sim}{\tau} \in\left(0, \min \left\{\frac{\sqrt{2}}{\kappa_{Y}}, \frac{1}{2}\right\}\right)$ be fixed, and $\left(\boldsymbol{E}_{c}^{k}, \boldsymbol{H}_{c}^{k}, \Phi_{c}^{k}\right)$ be the iterates of (3.17) with initial data $\left(\boldsymbol{E}^{0}, \boldsymbol{H}^{0}, \Phi^{0}\right) \in Y$ and step size $\tau \in(0, \stackrel{\circ}{\tau}]$. There is a constant $C_{o}>0$ with

$$
\begin{gathered}
\int_{Q}\left(\varepsilon\left|\boldsymbol{E}^{0}\right|^{2}+\mu\left|\boldsymbol{H}^{0}\right|^{2}+\mu\left|\Phi^{0}\right|^{2}\right) \mathrm{d} x \leq C_{o} \tau \sum_{k=1}^{N} \int_{Q}\left(\left|\boldsymbol{E}_{c}^{k}\right|^{2}+\left|\Phi_{c}^{k}\right|^{2}\right) \mathrm{d} x+C_{o} \tau^{3} \sum_{k=0}^{N-1}\left(\left\|B\left(\begin{array}{c}
\boldsymbol{E}_{c}^{k, 3} \\
\boldsymbol{H}_{c}^{k, 3} \\
0
\end{array}\right)\right\|^{2}+\|\|^{A}\left(\begin{array}{c}
\boldsymbol{E}_{c}^{k, 1} \\
\boldsymbol{H}_{c}^{k, 1} \\
0
\end{array}\right)\left\|^{2}\right\|^{2}\right. \\
+C_{o} \tau^{3} \sum_{k=0}^{N-1} \sum_{i=1}^{3}\left\|D_{i}\left(\begin{array}{c}
0 \\
\boldsymbol{H}_{c}^{k, 3+2 i} \\
\Phi_{c}^{k, 3+2 i}
\end{array}\right)\right\|^{2}
\end{gathered}
$$

Here, $N:=\max \{m \in \mathbb{N} \mid m \tau \leq 9 \stackrel{\circ}{\tau}\}$, and the constant $C_{o}$ depends only on $\varepsilon, \mu, \stackrel{\circ}{\tau}$ and $Q$.

Apart from the artificial variable $\Phi$, the interior observability estimate (4.13) is a time-discrete counterpart to Lemma 3.1 in [26]. The additional higher order terms on the right hand side are to stabilize the estimate in the spirit of $[25]$.

The proof of Theorem 4.2 will be concluded in Subsection 4.4. Here it is crucial to have appropriate Helmholtz decompositions for the electric and magnetic fields. According to (2.2), the latter can be represented as

$$
\mu \mathbf{H}_{c}^{k}=\operatorname{curl} \mathbf{J}^{k}+\nabla q^{k}, \quad k \in \mathbb{N}
$$

with $q^{k} \in \mathrm{H}^{1}(Q)$, and $\mathbf{J}^{k} \in \mathrm{H}^{1}(Q)^{3}$ satisfying $\operatorname{div} \mathbf{J}^{k}=0, \mathbf{J}^{k} \times \nu=0$ on $\partial Q$, and $\operatorname{curl} \mathbf{J}^{k} \in \mathrm{H}_{0}(\operatorname{div}, Q)$. Regarding the electric field, the next lemma establishes a different decomposition imposing boundary conditions on the curl-free part. The corresponding proof is inspired by Lemma 3.1 in [26].

Lemma 4.3. Let $k \in \mathbb{N}$. There is a unique function $\psi^{k} \in \mathrm{H}_{N}(\operatorname{curl}, \operatorname{div}, Q)$ with $\operatorname{div} \psi^{k} \in \mathrm{H}_{0}^{1}(Q)$, curl $\psi^{k} \in$ $\mathrm{H}_{T}(\operatorname{curl}, \operatorname{div}, Q)$, and

$$
\varepsilon \boldsymbol{E}_{c}^{k}=\operatorname{curl} \operatorname{curl} \psi^{k}-\nabla \operatorname{div} \psi^{k}
$$

Proof. The Lax-Milgram Lemma yields a unique function $\psi^{k} \in \mathrm{H}_{N}(\operatorname{curl}$, div, $Q)$ with

$$
\int_{Q}\left(\operatorname{curl} \psi^{k}\right) \cdot(\operatorname{curl} \psi)+\left(\operatorname{div} \psi^{k}\right)(\operatorname{div} \psi) \mathrm{d} x=\int_{Q} \varepsilon \mathbf{E}_{c}^{k} \cdot \psi \mathrm{d} x
$$

for all $\psi \in \mathrm{H}_{N}(\operatorname{curl}, \operatorname{div}, Q)$. Theorem 1.1 in $[6]$ then implies that the function $\operatorname{div} \psi^{k}$ belongs to $\mathrm{H}_{0}^{1}(Q)$, and an integration by parts consequently leads to the identity

$$
\int_{Q}\left(\operatorname{curl} \psi^{k}\right) \cdot(\operatorname{curl} \psi) \mathrm{d} x=\int_{Q}\left(\varepsilon \mathbf{E}_{c}^{k}+\nabla \operatorname{div} \psi^{k}\right) \cdot \psi \mathrm{d} x .
$$

Testing the last formula with functions $\psi \in \mathrm{H}_{0}^{1}(Q)^{3}$ shows that the function curl $\psi^{k}$ is an element of $\mathrm{H}(\mathrm{curl}, Q)$, and that the asserted representation is true. The boundary condition for curl $\psi^{k}$ is due to Remark 2.5 in Section I of $[15]$.

It is crucial that the above decomposition of the electric field is orthogonal in the $\mathrm{L}^{2}$-sense. 


\subsection{An estimate for the divergence-free part of the magnetic field approximations}

Let $\stackrel{\circ}{\tau} \in\left(0, \min \left\{\frac{\sqrt{2}}{\kappa Y}, \frac{1}{2}\right\}\right)$ be fixed, and $\alpha:[0,9 \stackrel{\circ}{\tau}] \rightarrow[0,1]$ be a smooth function that is supported in $\left[\frac{9}{4} \stackrel{\circ}{\tau}, \frac{27}{4} \stackrel{\circ}{\tau}\right]$, and satisfies $\alpha=1$ on $[3 \dot{\tau}, 6 \tau]$. We recall the number $N=\max \left\{m \in \mathbb{N} \mid m \tau \leq 9 \dot{\tau}^{\circ}\right\}$ from Theorem 4.2. The assumptions on $\alpha$ then imply the identities

$$
\alpha(0)=\alpha((N-1) \tau)=\alpha(N \tau)=0
$$

for all $\tau \in(0, \stackrel{\sim}{\tau}]$.

This subsection, being the first part of the proof for Theorem 4.2, is devoted to the following inequality for the divergence-free part of the magnetic field approximation.

Lemma 4.4. Let $\varepsilon, \mu$ satisfy (1.2), $\tau \in(0, \stackrel{\circ}{\tau}]$, and $\left(\boldsymbol{E}^{0}, \boldsymbol{H}^{0}, \Phi^{0}\right) \in Y$ be the initial data for (3.17). There is a constant $C_{c}=C_{c}(\varepsilon, \mu, \stackrel{\circ}{\tau}, Q)>0$ with

$$
\begin{aligned}
\sum_{k=0}^{N} \alpha(k \tau) \int_{Q} \boldsymbol{H}_{c}^{k} \cdot p_{\operatorname{curl}}\left(\mu \boldsymbol{H}_{c}^{k}\right) \mathrm{d} x \leq & \frac{1}{16\|\mu\|_{\infty}} \sum_{k=1}^{N-1}\left\|\operatorname{curl} \boldsymbol{J}^{k}\right\|_{\mathrm{L}^{2}}^{2}+C_{c} \sum_{k=1}^{N-1}\left(\left\|\boldsymbol{E}_{c}^{k}\right\|_{\mathrm{L}^{2}}^{2}+\left\|\Phi_{c}^{k}\right\|_{\mathrm{L}^{2}}^{2}\right) \\
& \left.+C_{c} \tau^{2} \sum_{k=0}^{N-1}\left(\| \begin{array}{c}
\boldsymbol{E}_{c}^{k, 1} \\
\boldsymbol{H}_{c}^{k, 1} \\
0
\end{array}\right)\left\|^{2}+\right\| B\left(\begin{array}{c}
\boldsymbol{E}_{c}^{k, 3} \\
\boldsymbol{H}_{c}^{k, 3} \\
0
\end{array}\right)\left\|^{2}+\sum_{i=1}^{3}\right\| D_{i}\left(\begin{array}{c}
0 \\
\boldsymbol{H}_{c}^{k, 3+2 i} \\
\Phi_{c}^{k, 3+2 i}
\end{array}\right) \|^{2}\right) .
\end{aligned}
$$

Proof. In view of the boundary condition $\mathbf{J}^{k} \times \nu=0$ on $\partial Q$, an integration by parts and (4.15) lead to the identities

$$
\sum_{k=0}^{N} \alpha(k \tau) \int_{Q} \mathbf{H}_{c}^{k} \cdot \operatorname{curl} \mathbf{J}^{k} \mathrm{~d} x=\sum_{k=0}^{N-1} \alpha(k \tau)\left(\int_{Q} \frac{1}{2} \operatorname{curl}\left(\mathbf{H}_{c}^{k}+\mathbf{H}_{c}^{k+1}\right) \cdot \mathbf{J}^{k} \mathrm{~d} x-\int_{Q} \frac{1}{2} \operatorname{curl}\left(\mathbf{H}_{c}^{k+1}-\mathbf{H}_{c}^{k}\right) \cdot \mathbf{J}^{k} \mathrm{~d} x\right) .
$$

Plugging in (4.9)-(4.10) and integrating again by parts, the formula

$$
\begin{aligned}
& \sum_{k=0}^{N} \alpha(k \tau) \int_{Q} \mathbf{H}_{c}^{k} \cdot \operatorname{curl} \mathbf{J}^{k} \mathrm{~d} x \\
&=\sum_{k=0}^{N-1} \alpha(k \tau)\left(\int_{Q} \frac{\varepsilon}{\tau}\left(\mathbf{E}_{c}^{k+1}-\mathbf{E}_{c}^{k}\right) \cdot \mathbf{J}^{k} \mathrm{~d} x-\frac{\tau}{2} \int_{Q}\left(\begin{array}{c}
\partial_{1} \frac{1}{\mu} \Phi_{c}^{k, 5} \\
\partial_{2} \frac{1}{\mu} \Phi_{c}^{k, 7} \\
\partial_{3} \frac{1}{\mu} \Phi_{c}^{k, 9}
\end{array}\right) \cdot \operatorname{curl} \mathbf{J}^{k} \mathrm{~d} x\right. \\
&\left.\quad-\int_{Q} \frac{1}{2} \operatorname{curl}\left(\mathbf{H}_{c}^{k+1}-\mathbf{H}_{c}^{k}\right) \cdot \mathbf{J}^{k} \mathrm{~d} x-\frac{\tau}{2} \int_{Q} \frac{1}{\mu}\left(\mathscr{C}_{2} \mathbf{E}_{c}^{k, 1}\right) \cdot\left(\mathscr{C}_{1} \mathbf{J}^{k}\right)-\frac{1}{\mu}\left(\mathscr{C}_{1} \mathbf{E}_{c}^{k, 3}\right) \cdot\left(\mathscr{C}_{2} \mathbf{J}^{k}\right) \mathrm{d} x\right)
\end{aligned}
$$

follows. Note that the integration by parts rule (3.12) is here valid due to the boundary conditions for $\mathbf{J}^{k}$. Summation by parts and the choice of $\alpha$ next yield the equation

$$
\begin{aligned}
\sum_{k=0}^{N} \alpha(k \tau) & \int_{Q} \mathbf{H}_{c}^{k} \cdot \operatorname{curl} \mathbf{J}^{k} \mathrm{~d} x \\
= & -\sum_{k=1}^{N-1} \int_{Q} \varepsilon \mathbf{E}_{c}^{k} \cdot \frac{1}{\tau}\left(\alpha(k \tau) \mathbf{J}^{k}-\alpha((k-1) \tau) \mathbf{J}^{k-1}\right) \mathrm{d} x-\sum_{k=0}^{N-1} \alpha(k \tau) \int_{Q} \frac{1}{2} \operatorname{curl}\left(\mathbf{H}_{c}^{k+1}-\mathbf{H}_{c}^{k}\right) \cdot \mathbf{J}^{k} \mathrm{~d} x \\
& -\frac{\tau}{2} \sum_{k=0}^{N-1} \alpha(k \tau) \int_{Q}\left(\begin{array}{c}
\partial_{1} \frac{1}{\mu} \Phi_{c}^{k, 5} \\
\partial_{2} \frac{1}{\mu} \Phi_{c}^{k, 7} \\
\partial_{3} \frac{1}{\mu} \Phi_{c}^{k, 9}
\end{array}\right) \cdot \operatorname{curl} \mathbf{J}^{k} \mathrm{~d} x-\frac{\tau}{2} \sum_{k=0}^{N-1} \alpha(k \tau) \int_{Q} \frac{1}{\mu}\left(\mathscr{C}_{2} \mathbf{E}_{c}^{k, 1}\right) \cdot\left(\mathscr{C}_{1} \mathbf{J}^{k}\right)-\frac{1}{\mu}\left(\mathscr{C}_{1} \mathbf{E}_{c}^{k, 3}\right) \cdot\left(\mathscr{C}_{2} \mathbf{J}^{k}\right) \mathrm{d} x .
\end{aligned}
$$

The four expressions on the right hand side are estimated in the subsequent steps.

(i) A simple algebraic manipulation of the first term on the right hand side of (4.16) shows the relation

$$
\sum_{k=1}^{N-1} \int_{Q} \varepsilon \mathbf{E}_{c}^{k} \cdot \frac{1}{\tau}\left(\alpha(k \tau) \mathbf{J}^{k}-\alpha((k-1) \tau) \mathbf{J}^{k-1}\right) \mathrm{d} x=\sum_{k=1}^{N-1} \int_{Q} \varepsilon \mathbf{E}_{c}^{k} \cdot \frac{1}{\tau}(\alpha(k \tau)-\alpha((k-1) \tau)) \mathbf{J}^{k} \mathrm{~d} x
$$




$$
+\sum_{k=1}^{N-1} \int_{Q} \varepsilon \mathbf{E}_{c}^{k} \cdot \frac{1}{\tau}\left(\mathbf{J}^{k}-\mathbf{J}^{k-1}\right) \alpha((k-1) \tau) \mathrm{d} x .
$$

To obtain an appropriate estimate for the first term on the right hand side of (4.17), we fix a number $\gamma>0$ that we determine at the end of the proof. Since $\operatorname{div} \mathbf{J}^{k}=0$, an application of inequality (2.1) to $\mathbf{J}^{k}$ then leads to the relations

$$
\begin{aligned}
\left|\sum_{k=1}^{N-1} \int_{Q} \varepsilon \mathbf{E}_{c}^{k} \cdot \frac{\alpha(k \tau)-\alpha((k-1) \tau)}{\tau} \mathbf{J}^{k} \mathrm{~d} x\right| & \leq \sum_{k=1}^{N-1}\left(\frac{\gamma}{2}\left\|\mathbf{J}^{k}\right\|_{\mathrm{L}^{2}}^{2}+\frac{1}{2 \gamma}\left\|\alpha^{\prime}\right\|_{\infty}^{2}\|\varepsilon\|_{\infty}^{2}\left\|\mathbf{E}_{c}^{k}\right\|_{\mathrm{L}^{2}}^{2}\right) \\
& \leq \sum_{k=1}^{N-1}\left(\frac{C_{T} \gamma}{2}\left\|\operatorname{curl} \mathbf{J}^{k}\right\|_{\mathrm{L}^{2}}^{2}+\frac{1}{2 \gamma}\left\|\alpha^{\prime}\right\|_{\infty}^{2}\|\varepsilon\|_{\infty}^{2}\left\|\mathbf{E}_{c}^{k}\right\|_{\mathrm{L}^{2}}^{2}\right) .
\end{aligned}
$$

We next deal with the second expression on the right hand side of (4.17), and obtain with (4.10) and (4.14) the difference equation

$$
\begin{aligned}
\frac{1}{\tau} \operatorname{curl}\left(\mathbf{J}^{k}-\mathbf{J}^{k-1}\right)+\frac{1}{\tau} \nabla\left(q^{k}-q^{k-1}\right)= & -\frac{1}{2} \operatorname{curl}\left(\mathbf{E}_{c}^{k}+\mathbf{E}_{c}^{k-1}\right)-\mu\left(\begin{array}{c}
\partial_{1} \frac{1}{\mu} \Phi_{c}^{k-1,5} \\
\partial_{2} \frac{1}{\mu} \Phi_{c}^{k-1,7} \\
\partial_{3} \frac{1}{\mu} \Phi_{c}^{k-1,9}
\end{array}\right)-\frac{\tau}{2} \mathscr{C}_{1} \frac{1}{\varepsilon} \mathscr{C}_{1} \mathbf{H}_{c}^{k-1,1} \\
& +\frac{\tau}{2} \mathscr{C}_{2} \frac{1}{\varepsilon} \mathscr{C}_{2} \mathbf{H}_{c}^{k-1,3} .
\end{aligned}
$$

By means of the orthogonal projection $p_{\text {curl }}$ onto the divergence-free part, we infer

$$
\frac{1}{\tau} \operatorname{curl}\left(\mathbf{J}^{k}-\mathbf{J}^{k-1}\right)=-\frac{1}{2} \operatorname{curl}\left(\mathbf{E}_{c}^{k}+\mathbf{E}_{c}^{k-1}\right)-p_{\text {curl }} \mu\left(\begin{array}{c}
\partial_{1} \frac{1}{\mu} \Phi_{c}^{k-1,5} \\
\partial_{2} \frac{1}{\mu} \Phi_{c}^{k-1,7} \\
\partial_{3} \frac{1}{\mu} \Phi_{c}^{k-1,9}
\end{array}\right)-\frac{\tau}{2} p_{\text {curl }}\left(\mathscr{C}_{1} \frac{1}{\varepsilon} \mathscr{C}_{1} \mathbf{H}_{c}^{k-1,1}-\mathscr{C}_{2} \frac{1}{\varepsilon} \mathscr{C}_{2} \mathbf{H}_{c}^{k-1,3}\right) .
$$

The Helmholtz-decomposition (2.2) further yields functions $\check{\varphi}_{1}^{k-1}, \check{\varphi}_{2}^{k-1} \in \mathrm{H}_{N}(\operatorname{curl}$, div, $Q)$ with

$$
\operatorname{curl} \check{\varphi}_{1}^{k-1}=p_{\operatorname{curl}}\left(\mathscr{C}_{1} \frac{1}{\varepsilon} \mathscr{C}_{1} \mathbf{H}_{c}^{k-1,1}-\mathscr{C}_{2} \frac{1}{\varepsilon} \mathscr{C}_{2} \mathbf{H}_{c}^{k-1,3}\right), \quad \operatorname{curl} \check{\varphi}_{2}^{k-1}=p_{\text {curl }} \mu\left(\begin{array}{c}
\partial_{1} \frac{1}{\mu} \Phi_{c}^{k-1,5} \\
\partial_{2} \frac{1}{\mu} \Phi_{c}^{k-1,7} \\
\partial_{3} \frac{1}{\mu} \Phi_{c}^{k-1,9}
\end{array}\right)
$$

and consequently Theorem 2.9 in Section I of [15] states the existence of a function $\eta^{k-1} \in \mathrm{H}^{1}(Q)$ with

$$
\frac{1}{\tau}\left(\mathbf{J}^{k}-\mathbf{J}^{k-1}\right)=-\frac{1}{2}\left(\mathbf{E}_{c}^{k}+\mathbf{E}_{c}^{k-1}\right)-\frac{\tau}{2} \check{\varphi}_{1}^{k-1}-\check{\varphi}_{2}^{k-1}-\nabla \eta^{k-1} .
$$

This relation in particular implies that the function $\nabla \eta^{k-1}$ belongs to $\mathrm{H}^{1}(Q)^{3}$ with $\nabla \eta^{k-1} \times \nu=0$ on $\partial Q$, meaning $\nabla \eta^{k-1}$ is orthogonal to the space $\operatorname{curl}(\mathrm{H}(\operatorname{curl}, Q))$. Let $\psi_{k}$ be given by Lemma 4.3 . Since $\operatorname{div} \mathbf{J}^{k}=0$, we moreover conclude that the expression $\int_{Q}\left(\nabla \operatorname{div} \psi^{k}\right) \cdot \frac{1}{\tau}\left(\mathbf{J}^{k}-\mathbf{J}^{k-1}\right) \mathrm{d} x$ vanishes. Altogether, Lemma 4.3 and (4.20) imply the identities

$$
\begin{aligned}
& \sum_{k=1}^{N-1} \alpha((k-1) \tau) \int_{Q} \varepsilon \mathbf{E}_{c}^{k} \cdot \frac{1}{\tau}\left(\mathbf{J}^{k}-\mathbf{J}^{k-1}\right) \mathrm{d} x=\sum_{k=1}^{N-1} \alpha((k-1) \tau) \int_{Q}\left(\operatorname{curl} \operatorname{curl} \psi^{k}\right) \cdot \frac{1}{\tau}\left(\mathbf{J}^{k}-\mathbf{J}^{k-1}\right) \mathrm{d} x \\
&=-\sum_{k=1}^{N-1} \alpha((k-1) \tau)\left(\int_{Q}\left(\operatorname{curl} \operatorname{curl} \psi^{k}\right) \cdot \frac{1}{2}\left(\mathbf{E}_{c}^{k}+\mathbf{E}_{c}^{k-1}\right) \mathrm{d} x+\frac{\tau}{2} \int_{Q}\left(\operatorname{curl} \operatorname{curl} \psi^{k}\right) \cdot \check{\varphi}_{1}^{k-1} \mathrm{~d} x\right. \\
&\left.+\int_{Q}\left(\operatorname{curl} \operatorname{curl} \psi^{k}\right) \cdot \check{\varphi}_{2}^{k-1} \mathrm{~d} x\right) .
\end{aligned}
$$

Since the decomposition in Lemma 4.3 is orthogonal and $0 \leq \alpha \leq 1$, the first summand on the right hand side of (4.21) is bounded according to the relation

$$
\left|\sum_{k=1}^{N-1} \alpha((k-1) \tau) \int_{Q}\left(\operatorname{curl} \operatorname{curl} \psi^{k}\right) \cdot \frac{1}{2}\left(\mathbf{E}_{c}^{k}+\mathbf{E}_{c}^{k-1}\right) \mathrm{d} x\right| \leq\|\varepsilon\|_{\infty} \sum_{k=1}^{N-1}\left\|\mathbf{E}_{c}^{k}\right\|_{L^{2}}^{2} .
$$


Considering the choice of $\check{\varphi}_{1}^{k-1}$ in (4.19) and Lemma 4.3, twice integrating by parts transforms the second expression on the right hand side of (4.21) into the form

$$
\begin{array}{r}
\frac{\tau}{2} \sum_{k=1}^{N-1} \alpha((k-1) \tau) \int_{Q}\left(\operatorname{curl} \operatorname{curl} \psi^{k}\right) \cdot \check{\varphi}_{1}^{k-1} \mathrm{~d} x=\frac{\tau}{2} \sum_{k=1}^{N-1} \alpha((k-1) \tau) \int_{Q}\left(\operatorname{curl} \psi^{k}\right) \cdot\left(\mathscr{C}_{1} \frac{1}{\varepsilon} \mathscr{C}_{1} \mathbf{H}_{c}^{k-1,1}-\mathscr{C}_{2} \frac{1}{\varepsilon} \mathscr{C}_{2} \mathbf{H}_{c}^{k-1,3}\right) \mathrm{d} x \\
=-\frac{\tau}{2} \sum_{k=1}^{N-1} \alpha((k-1) \tau) \int_{Q}\left(\left(\mathscr{C}_{2} \operatorname{curl} \psi^{k}\right) \cdot \frac{1}{\varepsilon} \mathscr{C}_{1} \mathbf{H}_{c}^{k-1,1}-\left(\mathscr{C}_{1} \operatorname{curl} \psi^{k}\right) \cdot \frac{1}{\varepsilon} \mathscr{C}_{2} \mathbf{H}_{c}^{k-1,3}\right) \mathrm{d} x
\end{array}
$$

Formula (3.12) is here applicable since the vectors $\left(\frac{1}{\varepsilon} \mathscr{C}_{1} \mathbf{H}_{c}^{k-1,1}, \frac{1}{\mu} \mathscr{C}_{2} \mathbf{E}_{c}^{k-1,1}, 0\right)$ and $\left(\frac{1}{\varepsilon} \mathscr{C}_{2} \mathbf{H}_{c}^{k-1,3}, \frac{1}{\mu} \mathscr{C}_{1} \mathbf{E}_{c}^{k-1,3}, 0\right)$ belong to $Y$ by Remark 4.1. Lemma 4.3 and (2.1) consequently cause the estimate

$$
\begin{gathered}
\left|\frac{\tau}{2} \sum_{k=1}^{N-1} \alpha((k-1) \tau) \int_{Q}\left(\operatorname{curl} \operatorname{curl} \psi^{k}\right) \cdot \check{\varphi}_{1}^{k-1} \mathrm{~d} x\right| \leq \sum_{k=2}^{N-1}\left(2 \frac{C_{T}}{\delta^{2}}\|\varepsilon\|_{\infty}^{2}\left\|\mathbf{E}_{c}^{k}\right\|_{\mathrm{L}^{2}}^{2}+\frac{\tau^{2}}{8}\left\|\mathscr{C}_{2} \mathbf{H}_{c}^{k-1,3}\right\|_{\mathrm{L}^{2}}^{2}\right. \\
\left.+\frac{\tau^{2}}{8}\left\|\mathscr{C}_{1} \mathbf{H}_{c}^{k-1,1}\right\|_{\mathrm{L}^{2}}^{2}\right) .
\end{gathered}
$$

Concerning the third term on the right hand side of (4.21), an integration by parts, (4.19), (4.11) and Lemma 4.3 establish the representation

$$
\begin{aligned}
\sum_{k=1}^{N-1} \alpha((k-1) \tau) \int_{Q}\left(\operatorname{curl} \operatorname{curl} \psi^{k}\right) \cdot \check{\varphi}_{2}^{k-1} \mathrm{~d} x= & \sum_{k=1}^{N-1} \alpha((k-1) \tau)\left(\int_{Q}\left(\operatorname{curl} \psi^{k}\right) \cdot\left(\mu \nabla \frac{1}{\mu} \Phi_{c}^{k-1,6}\right) \mathrm{d} x\right. \\
& \left.+\frac{\tau}{2} \int_{Q}\left(\operatorname{curl} \psi^{k}\right) \cdot \mu\left(\begin{array}{c}
\partial_{1} \frac{1}{\mu^{3}} \partial_{1} \mu \mathbf{H}_{c, 1}^{k-1,5} \\
-\partial_{2} \frac{1}{\mu^{3}} \partial_{2} \mu \mathbf{H}_{c, 2}^{k-1,7} \\
-2 \partial_{3} \frac{1}{\mu^{3}} \partial_{2} \mu \mathbf{H}_{c, 2}^{k-1,7}-\partial_{3} \frac{1}{\mu^{3}} \partial_{3} \mu \mathbf{H}_{c, 3}^{k-1,9}
\end{array}\right) \mathrm{d} x\right) .
\end{aligned}
$$

To estimate the right hand side of (4.24), we recall the boundary condition for curl $\psi^{k}$ due to Lemma 4.3, and the relations $\alpha(0)=0$ and $\Phi_{c}^{k-1,6}=\Phi_{c}^{k-1}-\frac{\tau}{\mu^{2}} \partial_{1} \mu \mathbf{H}_{c, 1}^{k-1,5}$ from (4.15) and (4.2)-(4.4). An integration by parts and (2.1) now yield the inequalities

$$
\begin{aligned}
& \left|\sum_{k=1}^{N-1} \alpha((k-1) \tau) \int_{Q}\left(\operatorname{curl} \operatorname{curl} \psi^{k}\right) \cdot \check{\varphi}_{2}^{k-1} \mathrm{~d} x\right| \\
& \leq \sum_{k=2}^{N-1} \alpha((k-1) \tau)\left(\left|\int_{Q}\left(\operatorname{curl} \psi^{k}\right) \cdot \frac{1}{\mu}(\nabla \mu) \Phi_{c}^{k-1,6} \mathrm{~d} x\right|+\frac{\tau}{2}\left|\int_{Q}\left(\operatorname{curl} \psi^{k}\right) \cdot \frac{1}{\mu^{2}}\left(\begin{array}{c}
\partial_{1}^{2} \mu \mathbf{H}_{c, 1}^{k-1,5} \\
-\partial_{2}^{2} \mu \mathbf{H}_{c, 2}^{k-1,7} \\
-2 \partial_{3} \partial_{2} \mu \mathbf{H}_{c, 2}^{k-1,7}-\partial_{3}^{2} \mu \mathbf{H}_{c, 3}^{k-1,9}
\end{array}\right) \mathrm{d} x\right|\right. \\
& \left.+\frac{3 \tau}{2}\left|\int_{Q}\left(\operatorname{curl} \psi^{k}\right) \cdot\left(\begin{array}{c}
\left(\frac{\partial_{1} \mu}{\mu^{3}}\right) \partial_{1} \mu \mathbf{H}_{c, 1}^{k-1,5} \\
-\left(\frac{\partial_{2} \mu}{\mu^{3}}\right) \partial_{2} \mu \mathbf{H}_{c, 2}^{k-1,7} \\
-2\left(\frac{\partial_{3} \mu}{\mu^{3}}\right) \partial_{2} \mu \mathbf{H}_{c, 2}^{k-1,7}-\left(\frac{\partial_{3} \mu}{\mu^{3}}\right) \partial_{3} \mu \mathbf{H}_{c, 3}^{k-1,9}
\end{array}\right) \mathrm{d} x\right|\right) \\
& \leq \sum_{k=2}^{N-1}\left(\frac{\|\nabla \mu\|_{\infty}\|\varepsilon\|_{\infty}^{2}}{\delta} C_{T}\left\|\mathbf{E}_{c}^{k}\right\|_{L^{2}}^{2}+\frac{\|\nabla \mu\|_{\infty}}{2 \delta}\left\|\Phi_{c}^{k-1}\right\|_{L^{2}}^{2}+\frac{\tau^{2}\|\nabla \mu\|_{\infty}}{2 \delta^{5}}\left\|\partial_{1} \mu \mathbf{H}_{c}^{k-1,5}\right\|_{L^{2}}^{2}\right. \\
& +\frac{\tau}{2}\left|\int_{Q}\left(\partial_{i} \frac{1}{\mu^{2}}\left(\operatorname{curl} \psi^{k}\right)_{i}\right)_{i=1}^{3} \cdot\left(\begin{array}{c}
\partial_{1} \mu \mathbf{H}_{c, 1}^{k-1,5} \\
-\partial_{2} \mu \mathbf{H}_{c, 2}^{k-1,7} \\
-2 \partial_{2} \mu \mathbf{H}_{c, 2}^{k-1,7}-\partial_{3} \mu \mathbf{H}_{c, 3}^{k-1,9}
\end{array}\right) \mathrm{d} x\right| \\
& \left.+9\left\|\operatorname{curl} \psi^{k}\right\|_{\mathrm{L}^{2}}^{2}+\tau^{2} \frac{\|\nabla \mu\|_{\infty}^{2}}{\delta^{6}} \sum_{i=1}^{3}\left\|\partial_{i} \mu \mathbf{H}_{c, i}^{k-1,3+2 i}\right\|_{\mathrm{L}^{2}}^{2}\right)
\end{aligned}
$$




$$
\leq \sum_{k=2}^{N-1}\left(\hat{C}_{T}\left\|\mathbf{E}_{c}^{k}\right\|_{\mathrm{L}^{2}}^{2}+\frac{\|\nabla \mu\|_{\infty}}{2 \delta}\left\|\Phi_{c}^{k-1}\right\|_{\mathrm{L}^{2}}^{2}+\hat{C}_{\mu} \tau^{2} \sum_{i=1}^{3}\left\|\partial_{i} \mu \mathbf{H}_{c, i}^{k-1,3+2 i}\right\|_{\mathrm{L}^{2}}^{2}\right),
$$

employing the two positive numbers

$$
\hat{C}_{T}:=C_{T}\|\varepsilon\|_{\infty}^{2}\left(\frac{\|\nabla \mu\|_{\infty}}{\delta}+\frac{2}{\delta^{4}}+8 \frac{\|\nabla \mu\|_{\infty}^{2}}{\delta^{6}}+9\right), \quad \hat{C}_{\mu}:=\|\nabla \mu\|_{\infty}\left(\frac{1}{\delta^{5}}+\frac{\|\nabla \mu\|_{\infty}^{2}}{\delta^{6}}\right)+1 .
$$

Summing up, (4.21)-(4.23) and (4.25) bound the second expression on the right hand side of (4.17) by the inequality

$$
\begin{aligned}
\left|\sum_{k=1}^{N-1} \alpha((k-1) \tau) \int_{Q} \varepsilon \mathbf{E}_{c}^{k} \cdot \frac{1}{\tau}\left(\mathbf{J}^{k}-\mathbf{J}^{k-1}\right) \mathrm{d} x\right| \leq \sum_{k=1}^{N-1}( & \tilde{C}_{T}\left\|\mathbf{E}_{c}^{k}\right\|_{\mathrm{L}^{2}}^{2}+\frac{\tau^{2}}{8}\left(\left\|\mathscr{C}_{2} \mathbf{H}_{c}^{k-1,3}\right\|_{\mathrm{L}^{2}}^{2}+\left\|\mathscr{C}_{1} \mathbf{H}_{c}^{k-1,1}\right\|_{\mathrm{L}^{2}}^{2}\right) \\
& \left.+\hat{C}_{\mu} \tau^{2} \sum_{i=1}^{3}\left\|\partial_{i} \mu \mathbf{H}_{c, i}^{k, 3+2 i}\right\|_{\mathrm{L}^{2}}^{2}+\frac{\|\nabla \mu\|_{\infty}}{2 \delta}\left\|\Phi_{c}^{k}\right\|_{\mathrm{L}^{2}}^{2}\right)
\end{aligned}
$$

with $\tilde{C}_{T}:=\|\varepsilon\|_{\infty}+2 \frac{C_{T}}{\delta^{2}}\|\varepsilon\|_{\infty}^{2}+\hat{C}_{T}$. Combining (4.17), (4.18) and (4.26), the estimate

$$
\begin{aligned}
& \left|\sum_{k=1}^{N-1} \int_{Q} \varepsilon \mathbf{E}_{c}^{k} \cdot \frac{1}{\tau}\left(\alpha(k \tau) \mathbf{J}^{k}-\alpha((k-1) \tau) \mathbf{J}^{k-1}\right) \mathrm{d} x\right| \\
& \leq \sum_{k=1}^{N-1}\left(\frac{C_{T} \gamma}{2}\left\|\operatorname{curl} \mathbf{J}^{k}\right\|_{\mathrm{L}^{2}}^{2}+\left(\frac{1}{2 \gamma}\left\|\alpha^{\prime}\right\|_{\infty}^{2}\|\varepsilon\|_{\infty}^{2}+\tilde{C}_{T}\right)\left\|\mathbf{E}_{c}^{k}\right\|_{\mathrm{L}^{2}}^{2}+\frac{\tau^{2}}{8}\left(\left\|\mathscr{C}_{2} \mathbf{H}_{c}^{k-1,3}\right\|_{\mathrm{L}^{2}}^{2}+\left\|\mathscr{C}_{1} \mathbf{H}_{c}^{k-1,1}\right\|_{\mathrm{L}^{2}}^{2}\right)\right. \\
& \left.\quad+\hat{C}_{\mu} \tau^{2} \sum_{i=1}^{3}\left\|\partial_{i} \mu \mathbf{H}_{c, i}^{k, 3+2 i}\right\|_{\mathrm{L}^{2}}^{2}+\frac{\|\nabla \mu\|_{\infty}}{2 \delta}\left\|\Phi_{c}^{k}\right\|_{\mathrm{L}^{2}}^{2}\right)
\end{aligned}
$$

directly follows.

(ii) We next deal with the second summand on the right hand side of (4.16). Employing the boundary condition $\mathbf{J}^{k} \times \nu=0$ on $\partial Q$ in an integration by parts, and plugging in (4.6), the relations

$$
\begin{gathered}
\left|\sum_{k=1}^{N-1} \alpha(k \tau) \int_{Q} \frac{1}{2} \operatorname{curl}\left(\mathbf{H}_{c}^{k+1}-\mathbf{H}_{c}^{k}\right) \cdot \mathbf{J}^{k} \mathrm{~d} x\right|=\frac{\tau}{2}\left|\sum_{k=1}^{N-1} \alpha(k \tau) \int_{Q}\left[-\left(\begin{array}{c}
\partial_{1} \frac{1}{\mu} \Phi_{c}^{k, 5} \\
\partial_{2} \frac{1}{\mu} \Phi_{c}^{k, 7} \\
\partial_{3} \frac{1}{\mu} \Phi_{c}^{k, 9}
\end{array}\right)+\frac{1}{\mu} \mathscr{C}_{2} \mathbf{E}_{c}^{k, 1}-\frac{1}{\mu} \mathscr{C}_{1} \mathbf{E}_{c}^{k, 3}\right] \cdot \operatorname{curl} \mathbf{J}^{k} \mathrm{~d} x\right| \\
\leq \sum_{k=1}^{N-1}\left(3 \gamma\left\|\operatorname{curl} \mathbf{J}^{k}\right\|_{\mathrm{L}^{2}}^{2}+\frac{\tau^{2}}{16 \gamma} \sum_{i=1}^{3}\left\|\partial_{i} \frac{1}{\mu} \Phi_{c}^{k, 3+2 i}\right\|_{\mathrm{L}^{2}}^{2}+\frac{\tau^{2}}{16 \gamma \delta^{2}}\left(\left\|\mathscr{C}_{2} \mathbf{E}_{c}^{k, 1}\right\|_{\mathrm{L}^{2}}^{2}+\left\|\mathscr{C}_{1} \mathbf{E}_{c}^{k, 3}\right\|_{\mathrm{L}^{2}}^{2}\right)\right)
\end{gathered}
$$

are derived.

(iii) Applying (2.1) for $\mathbf{J}^{k}$, the two remaining expressions on the right hand side of (4.16) are bounded according to

$$
\begin{gathered}
\frac{\tau}{2}\left|\sum_{k=1}^{N-1} \alpha(k \tau) \int_{Q}\left(\begin{array}{c}
\partial_{1} \frac{1}{\mu} \Phi_{c}^{k, 5} \\
\partial_{2} \frac{1}{\mu} \Phi_{c}^{k, 7} \\
\partial_{3} \frac{1}{\mu} \Phi_{c}^{k, 9}
\end{array}\right) \cdot \operatorname{curl} \mathbf{J}^{k} \mathrm{~d} x\right|+\frac{\tau}{2}\left|\sum_{k=1}^{N-1} \alpha(k \tau) \int_{Q} \frac{1}{\mu}\left(\mathscr{C}_{2} \mathbf{E}_{c}^{k, 1}\right) \cdot\left(\mathscr{C}_{1} \mathbf{J}^{k}\right)-\frac{1}{\mu}\left(\mathscr{C}_{1} \mathbf{E}_{c}^{k, 3}\right) \cdot\left(\mathscr{C}_{2} \mathbf{J}^{k}\right) \mathrm{d} x\right| \\
\quad \leq \sum_{k=1}^{N-1}\left(\frac{\tau^{2}}{16 \gamma} \sum_{i=1}^{3}\left\|\partial_{i} \frac{1}{\mu} \Phi_{c}^{k, 3+2 i}\right\|_{\mathrm{L}^{2}}^{2}+\gamma\left(1+\frac{C_{T}}{4}\right)\left\|\operatorname{curl} \mathbf{J}^{k}\right\|_{\mathrm{L}^{2}}^{2}+\frac{\tau^{2}}{2 \gamma \delta^{2}}\left(\left\|\mathscr{C}_{2} \mathbf{E}_{c}^{k, 1}\right\|_{\mathrm{L}^{2}}^{2}+\left\|\mathscr{C}_{1} \mathbf{E}_{c}^{k, 3}\right\|_{\mathrm{L}^{2}}^{2}\right)\right) .
\end{gathered}
$$

The desired estimate is now a consequence of (4.16), (4.27)-(4.29), and the choice $\gamma \leq\left(16\left(\frac{3}{4} C_{T}+4\right)\|\mu\|_{\infty}\right)^{-1}$. 


\subsection{An inequality involving the curl-free part of the magnetic field approximations}

The goal of this subsection is to establish an estimate for the gradient part of the magnetic field approximations from (3.17) which is similar to Lemma 4.4. The first step is to deduce a slight modification of a result in [16] which is well known to experts and of auxiliary character for our purposes. The lemma uses for a convenient representation of the gradient part of the magnetic field approximations.

Lemma 4.5. Let $q \in \mathrm{L}^{2}(Q)$ with $\int_{Q} q \mathrm{~d} x=0$. The constrained boundary value problem

$$
\begin{array}{rlrl}
\Delta w & =q & & \text { in } Q, \\
\frac{\partial w}{\partial \nu} & =0 & & \text { on } \partial Q, \\
\int_{Q} w \mathrm{~d} x & =0, &
\end{array}
$$

has a unique solution $w \in \mathrm{H}^{2}(Q)$. There further is a uniform constant $C_{G}=C_{G}(Q)>0$ with $\|w\|_{\mathrm{H}^{2}} \leq C_{G}\|q\|_{\mathrm{L}^{2}}$.

Proof. Denote the mean of a function $v \in \mathrm{H}^{1}(Q)$ on $Q$ by $[v]$, and consider the Hilbert space $V:=\{w \in$ $\left.\mathrm{H}^{1}(Q) \mid[w]=0\right\}$, equipped with the $\mathrm{H}^{1}$-norm. The Lax-Milgram Lemma and the generalized Poincaré inequality provide a unique function $w \in V$ with

$$
\int_{Q}(\nabla w) \cdot(\nabla v) \mathrm{d} x=-\int_{Q} q v \mathrm{~d} x
$$

for all $v \in V$. Since $[q]=0$ by assumption, a computation yields the formula

$$
\int_{Q}(\nabla w) \cdot(\nabla v) \mathrm{d} x=\int_{Q}(\nabla w) \cdot \nabla(v-[v]) \mathrm{d} x=-\int_{Q} q(v-[v]) \mathrm{d} x=-\int_{Q} q v \mathrm{~d} x
$$

for every function $v$ belonging to $\mathrm{H}^{1}(Q)$. As a result, $w$ is the unique solution of (4.30), and Theorem 3.2.1.3 of [16] implies that $w$ is an element of $\mathrm{H}^{2}(Q)$.

It remains to deduce the asserted estimate, what will be done by means of arguments from the proofs for Theorems 3.1.2.1 and 3.2.1.3 in [16]. Let $m \in \mathbb{N}$. Lemma 2.3.2 in [20] provides a convex set $Q_{m} \subseteq \mathbb{R}^{3}$ with a $\mathrm{C}^{2}$-boundary $\partial Q_{m}$ containing $Q$ and satisfying $\operatorname{dist}\left(\partial Q, \partial Q_{m}\right) \leq \frac{1}{m}$. The function $v:=w$ then also solves the problem

$$
-\Delta v+v=-q+w=: f \quad \text { in } Q, \quad \frac{\partial v}{\partial \nu}=0 \quad \text { on } \partial Q .
$$

This problem is again uniquely solvable by Theorem 3.2.1.3 in [16]. Let further $\tilde{f}$ denote the trivial extension of $f$ to $\mathbb{R}^{3}$. We consider for $m \in \mathbb{N}$ the problem

$$
-\Delta v_{m}+v_{m}=\tilde{f} \quad \text { in } Q_{m}, \quad \frac{\partial v_{m}}{\partial \nu}=0 \quad \text { on } \partial Q_{m},
$$

possessing a unique solution $v_{m} \in \mathrm{H}^{2}\left(Q_{m}\right)$ with $\left\|v_{m}\right\|_{\mathrm{H}^{2}\left(Q_{m}\right)} \leq \sqrt{6}\|\tilde{f}\|_{\mathrm{L}^{2}\left(Q_{m}\right)}=\sqrt{6}\|f\|_{\mathrm{L}^{2}(Q)}$, see Theorem 3.1.2.3 in [16]. The proof of Theorem 3.2.1.3 in [16] further yields a subsequence (still denoted by $\left(v_{m}\right)_{m}$ ) with $\left.v_{m}\right|_{Q} \rightarrow v=w$ weakly in $\mathrm{H}^{2}(Q)$ as $m \rightarrow \infty$. We hence conclude the estimates

$$
\sqrt{6}\|f\|_{\mathrm{L}^{2}(Q)} \geq \liminf _{m \rightarrow \infty}\left\|\left.v_{m}\right|_{Q}\right\|_{\mathrm{H}^{2}(Q)} \geq\|w\|_{\mathrm{H}^{2}(Q)} .
$$

An integration by parts further reveals the relations

$$
\int_{Q}|\nabla w|^{2} \mathrm{~d} x=-\int_{Q}(\Delta w) w \mathrm{~d} x=-\int_{Q} q w \mathrm{~d} x \leq\|q\|_{\mathrm{L}^{2}}\|w\|_{\mathrm{L}^{2}} .
$$

The asserted estimate now is a direct consequence of (4.31), (4.32) and the generalized Poincaré inequality.

The next lemma provides a relation involving the curl-free part of the magnetic field approximations obtained via (3.17). For the statement of the lemma, recall the orthogonal projection $p_{\nabla}$ associated to the Helmholtz decomposition (2.2), the fixed maximal step size $\stackrel{\circ}{\tau}$, and the cut-off function $\alpha$ from Subsection 4.2. 
Lemma 4.6. Let $\varepsilon, \mu$ satisfy (1.2), $\tau \in(0, \stackrel{\circ}{\tau}]$, and the initial data $\left(\boldsymbol{E}^{0}, \boldsymbol{H}^{0}, \Phi^{0}\right)$ for (3.17) belong to $Y$. There is a uniform constant $C_{\nabla}=C_{\nabla}(\varepsilon, \mu, \stackrel{\circ}{\tau}, Q)>0$ with

$$
\begin{aligned}
& \left|\sum_{k=0}^{N} \alpha(k \tau) \int_{Q} \boldsymbol{H}_{c}^{k} \cdot p_{\nabla}\left(\mu \boldsymbol{H}_{c}^{k}\right) \mathrm{d} x\right| \leq \frac{\delta}{16} \sum_{k=1}^{N-1}\left\|p_{\nabla}\left(\boldsymbol{H}_{c}^{k}\right)\right\|_{\mathrm{L}^{2}}^{2}+C_{\nabla} \sum_{k=1}^{N-1}\left(\left\|\Phi_{c}^{k}\right\|_{\mathrm{L}^{2}}^{2}+\left\|\boldsymbol{E}_{c}^{k}\right\|_{\mathrm{L}^{2}}^{2}\right) \\
& +C_{\nabla} \tau^{2} \sum_{k=1}^{N-1}\left(\left\|A\left(\begin{array}{c}
\boldsymbol{E}_{c}^{k, 1} \\
\boldsymbol{H}_{c}^{k, 1} \\
0
\end{array}\right)\right\|^{2}+\left\|B\left(\begin{array}{c}
\boldsymbol{E}_{c}^{k, 3} \\
\boldsymbol{H}_{c}^{k, 3} \\
0
\end{array}\right)\right\|^{2}+\sum_{i=1}^{3}\left\|D_{i}\left(\begin{array}{c}
0 \\
\boldsymbol{H}_{c}^{k, 3+2 i} \\
\Phi_{c}^{k, 3+2 i}
\end{array}\right)\right\|^{2}\right) .
\end{aligned}
$$

Proof. Relation (4.15) will again be employed several times without further notice. Applying the Helmholtz decomposition (2.2), the magnetic field approximation is represented by means of the formula

$$
\mathbf{H}_{c}^{k}=\operatorname{curl} \tilde{\mathbf{J}}^{k}+\nabla \tilde{q}^{k}
$$

where the function $\tilde{q}^{k} \in \mathrm{H}^{1}(Q)$ without loss of generality satisfies $\int_{Q} \tilde{q}^{k} \mathrm{~d} x=0$. As before, the field $\tilde{\mathbf{J}}^{k}$ belongs to $\mathrm{H}^{1}(Q)^{3}$ with $\operatorname{div} \tilde{\mathbf{J}}^{k}=0, \operatorname{curl} \tilde{\mathbf{J}}^{k} \in \mathrm{H}_{0}(\operatorname{div}, Q)$ and $\tilde{\mathbf{J}}^{k} \times \nu=0$ on $\partial Q$.

Employing first the boundary condition $\mathbf{H}_{c}^{k} \cdot \nu=0$ on $\partial Q$ in an integration by parts, the identities

$$
\begin{aligned}
\sum_{k=0}^{N} \alpha(k \tau) \int_{Q} p_{\nabla}\left(\mu \mathbf{H}_{c}^{k}\right) \cdot \mathbf{H}_{c}^{k} \mathrm{~d} x=\sum_{k=0}^{N} \alpha(k \tau) \int_{Q} \mu \mathbf{H}_{c}^{k} \cdot p_{\nabla}\left(\mathbf{H}_{c}^{k}\right) \mathrm{d} x \\
\quad=-\sum_{k=0}^{N-1}\left(\alpha(k \tau) \int_{Q} \frac{1}{2} \operatorname{div}\left(\mu\left(\mathbf{H}_{c}^{k}+\mathbf{H}_{c}^{k+1}\right)\right) \tilde{q}^{k} \mathrm{~d} x+\alpha(k \tau) \int_{Q} \frac{\mu}{2}\left(\mathbf{H}_{c}^{k+1}-\mathbf{H}_{c}^{k}\right) \cdot \nabla \tilde{q}^{k} \mathrm{~d} x\right)
\end{aligned}
$$

are derived. Formulas (4.12) and (4.6) further yield the representation

$$
\begin{aligned}
\sum_{k=0}^{N} \alpha(k \tau) \int_{Q} \mathbf{H}_{c}^{k} \cdot p_{\nabla}\left(\mu \mathbf{H}_{c}^{k}\right) \mathrm{d} x= & \sum_{k=0}^{N-1}\left(\alpha(k \tau) \int_{Q} \frac{\mu^{2}}{\tau}\left(\Phi_{c}^{k+1}-\Phi_{c}^{k}\right) \tilde{q}^{k} \mathrm{~d} x-\frac{\tau}{2} \alpha(k \tau) \int_{Q} \operatorname{div}\left(\mathscr{C}_{1} \mathbf{E}_{c}^{k, 3}-\mathscr{C}_{2} \mathbf{E}_{c}^{k, 1}\right) \tilde{q}^{k} \mathrm{~d} x\right. \\
& \left.+\frac{\tau}{2} \alpha(k \tau) \int_{Q} \mu\left(\begin{array}{c}
\partial_{1} \frac{1}{\mu} \Phi_{c}^{k, 5} \\
\partial_{2} \frac{1}{\mu} \Phi_{c}^{k, 7} \\
\partial_{3} \frac{1}{\mu} \Phi_{c}^{k, 9}
\end{array}\right) \cdot \nabla \tilde{q}^{k} \mathrm{~d} x+\frac{\tau}{2} \alpha(k \tau) \int_{Q}\left(\mathscr{C}_{1} \mathbf{E}_{c}^{k, 3}-\mathscr{C}_{2} \mathbf{E}_{c}^{k, 1}\right) \cdot \nabla \tilde{q}^{k} \mathrm{~d} x\right)
\end{aligned}
$$

Remark 4.1 shows that the vectors $\left(\frac{1}{\varepsilon} \mathscr{C}_{1} \mathbf{H}_{c}^{k, 1}, \frac{1}{\mu} \mathscr{C}_{2} \mathbf{E}_{c}^{k, 1}, 0\right)$ and $\left(\frac{1}{\varepsilon} \mathscr{C}_{2} \mathbf{H}_{c}^{k, 3}, \frac{1}{\mu} \mathscr{C}_{1} \mathbf{E}_{c}^{k, 3}, 0\right)$ belong to $Y$, and an integration by parts for the second term on the right hand side of the last relation thus leads to the equation

$$
\begin{aligned}
\sum_{k=0}^{N} \alpha(k \tau) \int_{Q} \mathbf{H}_{c}^{k} \cdot p_{\nabla}\left(\mu \mathbf{H}_{c}^{k}\right) \mathrm{d} x= & \sum_{k=0}^{N-1}\left(\alpha(k \tau) \int_{Q} \frac{\mu^{2}}{\tau}\left(\Phi_{c}^{k+1}-\Phi_{c}^{k}\right) \tilde{q}^{k} \mathrm{~d} x+\frac{\tau}{2} \alpha(k \tau) \int_{Q} \mu\left(\begin{array}{c}
\partial_{1} \frac{1}{\mu} \Phi_{c}^{k, 5} \\
\partial_{2} \frac{1}{\mu} \Phi_{c}^{k, 7} \\
\partial_{3} \frac{1}{\mu} \Phi_{c}^{k, 9}
\end{array}\right) \cdot \nabla \tilde{q}^{k} \mathrm{~d} x\right. \\
& \left.+\tau \alpha(k \tau) \int_{Q}\left(\mathscr{C}_{1} \mathbf{E}_{c}^{k, 3}-\mathscr{C}_{2} \mathbf{E}_{c}^{k, 1}\right) \cdot \nabla \tilde{q}^{k} \mathrm{~d} x\right)
\end{aligned}
$$

The right hand side of (4.34) is treated in the following two steps.

(i) Concerning the first expression on the right hand side of (4.34), a summation by parts leads to the result

$$
\sum_{k=0}^{N-1} \alpha(k \tau) \int_{Q} \frac{\mu^{2}}{\tau}\left(\Phi_{c}^{k+1}-\Phi_{c}^{k}\right) \tilde{q}^{k} \mathrm{~d} x=-\sum_{k=1}^{N-1} \int_{Q} \mu^{2} \Phi_{c}^{k}\left[\frac{\alpha(k \tau)-\alpha((k-1) \tau)}{\tau} \tilde{q}^{k}+\alpha((k-1) \tau) \frac{\tilde{q}^{k}-\tilde{q}^{k-1}}{\tau}\right] \mathrm{d} x .
$$

As in the proof of Lemma 4.4, we fix a number $\gamma>0$ that will be determined later. Since $\tilde{q}^{k}$ is chosen to have zero mean, the generalized Poincaré estimate provides the bound

$$
\left|\sum_{k=1}^{N-1} \int_{Q} \mu^{2} \Phi_{c}^{k} \frac{\alpha(k \tau)-\alpha((k-1) \tau)}{\tau} \tilde{q}^{k} \mathrm{~d} x\right| \leq \sum_{k=1}^{N-1}\left(\frac{\gamma}{2} C_{P}\|\mu\|_{\infty}^{4}\left\|\nabla \tilde{q}^{k}\right\|_{L^{2}}^{2}+\frac{1}{2 \gamma}\left\|\alpha^{\prime}\right\|_{\infty}^{2}\left\|\Phi_{c}^{k}\right\|_{L^{2}}^{2}\right)
$$


for the first term on the right hand side of (4.35), where $C_{P}>0$ is a uniform constant from the Poincaré inequality on $Q$.

We next estimate the second summand on the right hand side of (4.35). Applying the orthogonal projection $p_{\nabla}$ to (4.10), we first infer with (4.33) and (4.11) the formula

$$
\begin{aligned}
\frac{1}{\tau} \nabla\left(\tilde{q}^{k+1}-\tilde{q}^{k}\right)= & -\nabla \frac{1}{\mu} \Phi_{c}^{k, 6}-\frac{\tau}{2} p_{\nabla}\left(\begin{array}{c}
\partial_{1} \frac{1}{\mu^{3}} \partial_{1} \mu \mathbf{H}_{c, 1}^{k, 5} \\
-\partial_{2} \frac{1}{\mu^{3}} \partial_{2} \mu \mathbf{H}_{c, 2}^{k, 7} \\
-2 \partial_{3} \frac{1}{\mu^{3}} \partial_{2} \mu \mathbf{H}_{c, 2}^{k, 7}-\partial_{3} \frac{1}{\mu^{3}} \partial_{3} \mu \mathbf{H}_{c, 3}^{k, 9}
\end{array}\right)-p_{\nabla}\left(\frac{1}{2 \mu} \operatorname{curl}\left(\mathbf{E}_{c}^{k+1}+\mathbf{E}_{c}^{k}\right)\right) \\
& +\frac{\tau}{2} p_{\nabla}\left(\frac{1}{\mu}\left(\mathscr{C}_{2} \frac{1}{\varepsilon} \mathscr{C}_{2} \mathbf{H}_{c}^{k, 3}-\mathscr{C}_{1} \frac{1}{\varepsilon} \mathscr{C}_{1} \mathbf{H}_{c}^{k, 1}\right)\right) .
\end{aligned}
$$

Lemma 4.5 further yields the unique solution $\eta^{k} \in \mathrm{H}^{2}(Q)$ of $(4.30)$ with right hand side $q:=\mu^{2} \Phi_{c}^{k}-\frac{1}{|Q|} \int_{Q} \mu^{2} \Phi_{c}^{k} \mathrm{~d} x$. The vanishing mean of $\tilde{q}^{k}$ consequently leads to the identity

$$
\sum_{k=1}^{N-1} \alpha((k-1) \tau) \int_{Q} \mu^{2} \Phi_{c}^{k} \frac{\tilde{q}^{k}-\tilde{q}^{k-1}}{\tau} \mathrm{d} x=\sum_{k=1}^{N-1} \alpha((k-1) \tau) \int_{Q}\left(\Delta \eta^{k}\right) \frac{\tilde{q}^{k}-\tilde{q}^{k-1}}{\tau} \mathrm{d} x .
$$

In view of the boundary condition $\frac{\partial \eta^{k}}{\partial \nu}=0$ on $\partial Q$ and (4.37), an integration by parts on the right hand side of (4.38) thus shows the equation

$$
\begin{aligned}
\sum_{k=1}^{N-1} \alpha((k-1) \tau) \int_{Q} \mu^{2} \Phi_{c}^{k} \frac{\tilde{q}^{k}-\tilde{q}^{k-1}}{\tau} \mathrm{d} x= & \sum_{k=1}^{N-1}\left(\alpha((k-1) \tau) \int_{Q}\left(\nabla \eta^{k}\right) \cdot\left(\nabla \frac{1}{\mu} \Phi_{c}^{k-1,6}\right) \mathrm{d} x\right. \\
& +\frac{\tau}{2} \alpha((k-1) \tau) \int_{Q}\left(\nabla \eta^{k}\right) \cdot\left(\begin{array}{c}
\partial_{1} \frac{1}{\mu^{3}} \partial_{1} \mu \mathbf{H}_{c, 1}^{k-1,5} \\
-\partial_{2} \frac{1}{\mu^{3}} \partial_{2} \mu \mathbf{H}_{c, 2}^{k-1,7} \\
-2 \partial_{3} \frac{1}{\mu^{3}} \partial_{2} \mu \mathbf{H}_{c, 2}^{k-1,7}-\partial_{3} \frac{1}{\mu^{3}} \partial_{3} \mu \mathbf{H}_{c, 3}^{k-1,9}
\end{array}\right) \mathrm{d} x \\
& +\alpha((k-1) \tau) \int_{Q}\left(\nabla \eta^{k}\right) \cdot \frac{1}{2 \mu} \operatorname{curl}\left(\mathbf{E}_{c}^{k}+\mathbf{E}_{c}^{k-1}\right) \mathrm{d} x \\
& \left.-\frac{\tau}{2} \alpha((k-1) \tau) \int_{Q} \frac{1}{\mu}\left(\nabla \eta^{k}\right) \cdot\left(\mathscr{C}_{2} \frac{1}{\varepsilon} \mathscr{C}_{2} \mathbf{H}_{c}^{k-1,3}-\mathscr{C}_{1} \frac{1}{\varepsilon} \mathscr{C}_{1} \mathbf{H}_{c}^{k-1,1}\right) \mathrm{d} x\right) . \quad(4.39)
\end{aligned}
$$

All terms on the right hand side are next integrated by parts, employing that $\frac{\partial \eta^{k}}{\partial \nu}=0$ and $\mathbf{E}_{c}^{k} \times \nu=0=$ $\left(\frac{1}{\varepsilon} \mathscr{C}_{1} \mathbf{H}_{c}^{k, 1}\right) \times \nu=\left(\frac{1}{\varepsilon} \mathscr{C}_{2} \mathbf{H}_{c}^{k, 3}\right) \times \nu$ on $\partial Q$, see Remark 4.1. In particular, formula (3.12) applies for the fourth integral on the right hand side of (4.39). Using the identity $\Phi_{c}^{k-1,6}=\Phi_{c}^{k-1}-\frac{\tau}{\mu^{2}} \partial_{1} \mu \mathbf{H}_{c, 1}^{k-1,5}$, we eventually arrive at the inequality

$$
\begin{aligned}
\left|\sum_{k=1}^{N-1} \alpha((k-1) \tau) \int_{Q} \mu^{2} \Phi_{c}^{k} \frac{\tilde{q}^{k}-\tilde{q}^{k-1}}{\tau} \mathrm{d} x\right| \leq & \frac{1}{2} \sum_{k=1}^{N}\left\|\mathbf{E}_{c}^{k}\right\|_{\mathrm{L}^{2}}^{2}+\sum_{k=1}^{N-1} C_{\mu}\left(\left\|\Phi_{c}^{k}\right\|_{\mathrm{L}^{2}}^{2}+\left\|\eta^{k}\right\|_{\mathrm{H}^{2}}^{2}\right) \\
& +\sum_{k=1}^{N-1}\left(C_{\mu} \tau^{2} \sum_{i=1}^{3}\left\|\partial_{i} \mu \mathbf{H}_{c, i}^{k, 3+2 i}\right\|_{\mathrm{L}^{2}}^{2}+\frac{\tau^{2}}{4 \delta^{2}}\left(\left\|\mathscr{C}_{2} \mathbf{H}_{c}^{k, 3}\right\|_{\mathrm{L}^{2}}^{2}+\left\|\mathscr{C}_{1} \mathbf{H}_{c}^{k, 1}\right\|_{\mathrm{L}^{2}}^{2}\right)\right)
\end{aligned}
$$

where $C_{\mu}=C_{\mu}(\mu)>0$ is a constant. By means of the inequality

$$
\left\|\eta^{k}\right\|_{\mathrm{H}^{2}} \leq C_{G}\left\|\mu^{2} \Phi_{c}^{k}-\frac{1}{|Q|} \int_{Q} \mu^{2} \Phi_{c}^{k} \mathrm{~d} y\right\|_{\mathrm{L}^{2}} \leq 2 C_{G}\|\mu\|_{\infty}^{2}\left\|\Phi_{c}^{k}\right\|_{\mathrm{L}^{2}}
$$

see Lemma 4.5, we infer from (4.35), (4.36) and (4.40) the estimate

$$
\left|\sum_{k=0}^{N-1} \alpha(k \tau) \int_{Q} \frac{\mu^{2}}{\tau}\left(\Phi_{c}^{k+1}-\Phi_{c}^{k}\right) \tilde{q}^{k} \mathrm{~d} x\right| \leq \sum_{k=1}^{N-1}\left(\frac{\gamma}{2} C_{P}\|\mu\|_{\infty}^{4}\left\|\nabla \tilde{q}^{k}\right\|_{\mathrm{L}^{2}}^{2}+\left(\frac{1}{2 \gamma}\left\|\alpha^{\prime}\right\|_{\infty}^{2}+\tilde{C}_{\mu}\right)\left\|\Phi_{c}^{k}\right\|_{\mathrm{L}^{2}}^{2}\right)+\frac{1}{2} \sum_{k=1}^{N}\left\|\mathbf{E}_{c}^{k}\right\|_{\mathrm{L}^{2}}^{2}
$$




$$
+\sum_{k=1}^{N-1}\left(C_{\mu} \tau^{2} \sum_{i=1}^{3}\left\|\partial_{i} \mu \mathbf{H}_{c, i}^{k, 3+2 i}\right\|_{\mathrm{L}^{2}}^{2}+\frac{\tau^{2}}{4 \delta^{2}}\left(\left\|\mathscr{C}_{2} \mathbf{H}_{c}^{k, 3}\right\|_{\mathrm{L}^{2}}^{2}+\left\|\mathscr{C}_{1} \mathbf{H}_{c}^{k, 1}\right\|_{\mathrm{L}^{2}}^{2}\right)\right),
$$

where the number $\tilde{C}_{\mu}>0$ only depends on $\mu$ and $Q$. We have thus bounded the first term on the right hand side of (4.34).

(ii) Applying Young's inequality and the relation $\alpha(0)=0$ to the two remaining expressions in (4.34) leads to the result

$$
\begin{aligned}
& \left|\sum_{k=0}^{N-1} \frac{\tau}{2} \alpha(k \tau)\left(\int_{Q} \mu\left(\begin{array}{c}
\partial_{1} \frac{1}{\mu} \Phi_{c}^{k, 5} \\
\partial_{2} \frac{1}{\mu} \Phi_{c}^{k, 7} \\
\partial_{3} \frac{1}{\mu} \Phi_{c}^{k, 9}
\end{array}\right) \cdot \nabla \tilde{q}^{k} \mathrm{~d} x+2 \int_{Q}\left(\mathscr{C}_{1} \mathbf{E}_{c}^{k, 3}-\mathscr{C}_{2} \mathbf{E}_{c}^{k, 1}\right) \cdot \nabla \tilde{q}^{k} \mathrm{~d} x\right)\right| \\
& \leq \sum_{k=1}^{N-1}\left(5 \gamma\left\|\nabla \tilde{q}^{k}\right\|_{\mathrm{L}^{2}}^{2}+\frac{\tau^{2}\|\mu\|_{\infty}^{2}}{16 \gamma} \sum_{i=1}^{3}\left\|\partial_{i} \frac{1}{\mu} \Phi_{c}^{k, 3+2 i}\right\|_{\mathrm{L}^{2}}^{2}+\frac{\tau^{2}}{\gamma}\left(\left\|\mathscr{C}_{1} \mathbf{E}_{c}^{k, 3}\right\|_{\mathrm{L}^{2}}^{2}+\left\|\mathscr{C}_{2} \mathbf{E}_{c}^{k, 1}\right\|_{\mathrm{L}^{2}}^{2}\right)\right) .
\end{aligned}
$$

Choosing finally $\gamma \leq \delta\left(16\left(\frac{C_{P}}{2}\|\mu\|_{\infty}^{4}+5\right)\right)^{-1}$, we infer from (4.34), (4.41) and (4.42) the asserted estimate.

\subsection{Conclusion of the observability inequality}

Lemmas 4.4 and 4.6 now directly lead to the asserted observability estimate for scheme (3.17). It is crucial for the following proof that (3.17) is energy conserving.

Proof of Theorem 4.2. We first take the sum of the estimates from Lemmas 4.4 and 4.6. Using $\mu \geq \delta$, it follows

$$
\begin{aligned}
\sum_{k=0}^{N} \alpha(k \tau) \int_{Q} \mu\left|\mathbf{H}_{c}^{k}\right|^{2} \mathrm{~d} x \leq & \frac{1}{8} \sum_{k=1}^{N-1} \int_{Q} \mu\left|\mathbf{H}_{c}^{k}\right|^{2} \mathrm{~d} x+\left(C_{c}+C_{\nabla}\right)\left(\sum_{k=1}^{N-1}\left(\left\|\mathbf{E}_{c}^{k}\right\|_{\mathrm{L}^{2}}^{2}+\left\|\Phi_{c}^{k}\right\|_{\mathrm{L}^{2}}^{2}\right)\right. \\
& \left.\quad+\tau^{2} \sum_{k=0}^{N-1}\left(\left\|\left(\begin{array}{c}
\mathbf{E}_{c}^{k, 1} \\
\mathbf{H}_{c}^{k, 1} \\
0
\end{array}\right)\right\|^{2}+\left\|B\left(\begin{array}{c}
\mathbf{E}_{c}^{k, 3} \\
\mathbf{H}_{c}^{k, 3} \\
0
\end{array}\right)\right\|^{2}+\sum_{i=1}^{3}\left\|D_{i}\left(\begin{array}{c}
0 \\
\mathbf{H}_{c}^{k, 3+2 i} \\
\Phi_{c}^{k, 3+2 i}
\end{array}\right)\right\|^{2}\right)\right) .
\end{aligned}
$$

Employing on the other hand that $\alpha=1$ on $\left[3 \tau^{\circ}, 6{ }^{\circ}\right]$ and that (3.17) is energy conserving, we deduce the relations

$$
\begin{aligned}
\sum_{k=1}^{N} \int_{Q}\left(\mu\left|\mathbf{H}_{c}^{k}\right|^{2}+\varepsilon\left|\mathbf{E}_{c}^{k}\right|^{2}+\mu\left|\Phi_{c}^{k}\right|^{2}\right) \mathrm{d} x & \leq 4 \sum_{k=\left\lceil\frac{3 \tau}{\tau}\right\rceil}^{\left\lfloor\frac{6 \tau}{\tau}\right\rfloor} \int_{Q}\left(\mu\left|\mathbf{H}_{c}^{k}\right|^{2}+\varepsilon\left|\mathbf{E}_{c}^{k}\right|^{2}+\mu\left|\Phi_{c}^{k}\right|^{2}\right) \mathrm{d} x \\
& \leq 4 \sum_{k=1}^{N}\left(\alpha(k \tau) \int_{Q} \mu\left|\mathbf{H}_{c}^{k}\right|^{2} \mathrm{~d} x+\int_{Q} \varepsilon\left|\mathbf{E}_{c}^{k}\right|^{2}+\mu\left|\Phi_{c}^{k}\right|^{2} \mathrm{~d} x\right) .
\end{aligned}
$$

Plugging (4.43) into (4.44) and rearranging terms, we obtain the result

$$
\begin{array}{r}
\sum_{k=1}^{N} \int_{Q}\left(\mu\left|\mathbf{H}_{c}^{k}\right|^{2}+\varepsilon\left|\mathbf{E}_{c}^{k}\right|^{2}+\mu\left|\Phi_{c}^{k}\right|^{2}\right) \mathrm{d} x \leq 8\left(C_{c}+C_{\nabla}+\|\varepsilon\|_{\infty}\right) \sum_{k=1}^{N}\left\|\mathbf{E}_{c}^{k}\right\|_{\mathrm{L}^{2}}^{2}+8\left(C_{c}+C_{\nabla}+\|\mu\|_{\infty}\right) \sum_{k=1}^{N}\left\|\Phi_{c}^{k}\right\|_{\mathrm{L}^{2}}^{2} \\
\left.+8\left(C_{c}+C_{\nabla}\right) \tau^{2} \sum_{k=0}^{N-1}\left(\| \begin{array}{c}
(4.45) \\
\mathbf{E}_{c}^{k, 1} \\
\mathbf{H}_{c}^{k, 1} \\
0
\end{array}\right)\left\|^{2}+\right\| B\left(\begin{array}{c}
\mathbf{E}_{c}^{k, 3} \\
\mathbf{H}_{c}^{k, 3} \\
0
\end{array}\right)\left\|^{2}+\sum_{i=1}^{3}\right\| D_{i}\left(\begin{array}{c}
0 \\
\mathbf{H}_{c}^{k, 3+2 i} \\
\Phi_{c}^{k, 3+2 i}
\end{array}\right) \|^{2}\right) .
\end{array}
$$

Energy conservation also yields

$$
\int_{Q}\left(\mu\left|\mathbf{H}^{0}\right|^{2}+\varepsilon\left|\mathbf{E}^{0}\right|^{2}+\mu\left|\Phi^{0}\right|^{2}\right) \mathrm{d} x=\frac{1}{N} \sum_{k=1}^{N} \int_{Q}\left(\mu\left|\mathbf{H}_{c}^{k}\right|^{2}+\varepsilon\left|\mathbf{E}_{c}^{k}\right|^{2}+\mu\left|\Phi_{c}^{k}\right|^{2}\right) \mathrm{d} x .
$$

The relation $N \tau>8 \dot{\tau}^{\circ}$ and (4.45) then imply asserted observability estimate (4.13). 


\section{Exponential Stability of The DAMPED SCHEME}

In three steps we deduce the uniform exponential stability of scheme (3.18) from the observability inequality for (3.17). A formula describing the dissipation of the energy is derived in Subsection 5.1 for the iterates of (3.18). Differences between certain substeps of the damped and undamped schemes are afterwards estimated in Subsection 5.2. The exponential stability, see Theorem 3.9, is finally proved in Subsection 5.3 by combining Theorem 4.2 with the results of Subsections 5.1 and 5.2. A similar strategy has already been used for the one-dimensional wave equation in [30].

\subsection{An energy identity for the damped ADI scheme}

As in (4.1) we first introduce a convenient substep formalism for the damped scheme. Let $n \in \mathbb{N}_{0}, i \in\{1,2,3\}$, $\stackrel{\tau}{\tau}\left(0, \min \left\{\frac{1}{2}, \frac{\sqrt{2}}{\kappa_{Y}}\right\}\right)$ fixed, $\tau \in(0, \stackrel{\circ}{\tau}]$, and take initial data $\left(\mathbf{E}^{0}, \mathbf{H}^{0}, \Phi^{0}\right) \in Y$. Define then the substeps

$$
\begin{aligned}
&\left(\begin{array}{l}
\mathbf{E}^{n, 1} \\
\mathbf{H}^{n, 1} \\
\Phi^{n, 1}
\end{array}\right):=V_{\tau}(A)\left(\begin{array}{l}
\mathbf{E}^{n} \\
\mathbf{H}^{n} \\
\Phi^{n}
\end{array}\right) ; \\
&\left(\begin{array}{l}
\mathbf{E}^{n, 4} \\
\mathbf{H}^{n, 4} \\
\Phi^{n, 4}
\end{array}\right):=V_{\tau}(B)\left(\begin{array}{l}
\mathbf{E}^{n, 2} \\
\mathbf{E}^{n, 2} \\
\mathbf{H}^{n, 3} \\
\Phi^{n, 2}
\end{array}\right) ;\left(\begin{array}{l}
\mathbf{E}^{n, 5} \\
\mathbf{H}^{n, 5} \\
\Phi^{n, 5}
\end{array}\right):=\left(I-\frac{\tau}{2} B\right)^{-1}\left(\begin{array}{l}
\mathbf{E}^{n, 1} \\
\mathbf{E}^{n, 1} \\
\Phi^{n, 1}
\end{array}\right) ;\left(\begin{array}{l}
\mathbf{E}^{n, 4} \\
\mathbf{H}^{n, 4} \\
\Phi^{n, 4}
\end{array}\right) ; \quad\left(\begin{array}{l}
\mathbf{E}^{n, 2} \\
\Phi^{n, 3}
\end{array}\right):=\left(I+\frac{\tau}{2} A\right)\left(\begin{array}{l}
\mathbf{E}^{n, 6} \\
\mathbf{H}^{n, 2} \\
\Phi^{n, 2}
\end{array}\right) ; \\
&\left(\begin{array}{l}
\mathbf{E}^{n, 4+3 i} \\
\mathbf{H}^{n, 4+3 i} \\
\Phi^{n, 4+3 i}
\end{array}\right):=V_{\tau}\left(D_{i}\right)\left(\begin{array}{l}
\mathbf{E}^{n, 5} \\
\mathbf{H}^{n, 3+3 i} \\
\Phi^{n, 3+3 i}
\end{array}\right) ; \quad\left(\begin{array}{l}
\mathbf{E}^{n, 5+3 i} \\
\mathbf{H}^{n, 5+3 i} \\
\Phi^{n, 5+3 i}
\end{array}\right) ;=\left(I-\frac{\tau}{2} D_{i}\right)^{-1}\left(\begin{array}{l}
\mathbf{E}^{n, 4+3 i} \\
\mathbf{H}^{n, 4+3 i} \\
\Phi^{n, 4+3 i}
\end{array}\right) ; \\
&\left(\begin{array}{l}
\mathbf{E}^{n, 6+3 i} \\
\mathbf{H}^{n, 6+3 i} \\
\Phi^{n, 6+3 i}
\end{array}\right):=\left(I+\frac{\tau}{2} D_{i}\right)\left(\begin{array}{l}
\mathbf{E}^{n, 5+3 i} \\
\mathbf{H}^{n, 5+3 i} \\
\Phi^{n, 5+3 i}
\end{array}\right) .
\end{aligned}
$$

As in Remark 4.1, we conclude from Lemmas 3.11 and 3.16, Corollary 3.15, and the assumptions (1.2) and (3.2), that all substeps and the next iterate of (3.18) remain in $Y$. This observation will become important at the end of the proof for Theorem 3.9.

To find a useful identity for the energy of the iterates of (3.18), relations between the substeps in (5.1) are crucial. The last intermediate step for instance satisfies the formula

$$
\left\|\left(\begin{array}{c}
\mathrm{e}^{\tau \sigma} \mathbf{E}^{n+1} \\
\mathbf{H}^{n+1} \\
\mathrm{e}^{\tau \eta} \Phi^{n+1}
\end{array}\right)\right\|=\left\|\left(\begin{array}{c}
\mathbf{E}^{n, 15} \\
\mathbf{H}^{n, 15} \\
\Phi^{n, 15}
\end{array}\right)\right\| .
$$

All substeps with counter index being a multiple of 3 fulfill the identity

$$
\left\|\left(\begin{array}{c}
\mathbf{E}^{n, 3 k} \\
\mathbf{H}^{n, 3 k} \\
\Phi^{n, 3 k}
\end{array}\right)\right\|=\left\|\left(\begin{array}{c}
\mathbf{E}^{n, 3 k-2} \\
\mathbf{H}^{n, 3 k-2} \\
\Phi^{n, 3 k-2}
\end{array}\right)\right\|, \quad k \in\{1,2,3,4,5\},
$$

since the Cayley-Transform $S_{\tau}(L)$ is an isometry for $L \in\left\{A, B, D_{1}, D_{2}, D_{3}\right\}$. We also define

$$
\begin{aligned}
&\left(\begin{array}{c}
\check{\mathbf{E}}^{n, 1} \\
\check{\mathbf{H}}^{n, 1} \\
\check{\Phi}^{n, 1}
\end{array}\right):=\left(I-\frac{\tau^{2}}{4} A^{2}\right)^{-1}\left(\begin{array}{c}
\mathbf{E}^{n, 1} \\
\mathbf{H}^{n, 1} \\
\Phi^{n, 1}
\end{array}\right), \quad\left(\begin{array}{c}
\check{\mathbf{E}}^{n, 4} \\
\check{\mathbf{H}}^{n, 4} \\
\check{\Phi}^{n, 4}
\end{array}\right):=\left(I-\frac{\tau^{2}}{4} B^{2}\right)^{-1}\left(\begin{array}{c}
\mathbf{E}^{n, 4} \\
\mathbf{H}^{n, 4} \\
\Phi^{n, 4}
\end{array}\right), \\
&\left(\begin{array}{c}
\check{\mathbf{E}}^{n, 4+3 i} \\
\check{\mathbf{H}}^{n, 4+3 i} \\
\check{\Phi}^{n, 4+3 i}
\end{array}\right):=\left(I-\frac{\tau^{2}}{4} D_{i}^{2}\right)^{-1}\left(\begin{array}{c}
\mathbf{E}^{n, 4+3 i} \\
\mathbf{H}^{n, 4+3 i} \\
\Phi^{n, 4+3 i}
\end{array}\right) .
\end{aligned}
$$


Employing the skewadjointness of the operators $A, B, D_{1}, D_{2}$, and $D_{3}$, we arrive at the energy identity

$$
\begin{aligned}
&\left\|\left(\begin{array}{c}
\mathbf{E}^{n+1} \\
\mathbf{H}^{n+1} \\
\Phi^{n+1}
\end{array}\right)\right\|^{2}-\left\|\left(\begin{array}{c}
\mathbf{E}^{n} \\
\mathbf{H}^{n} \\
\Phi^{n}
\end{array}\right)\right\|^{2} \\
&=-\left(\mathrm{e}^{2 \tau \sigma}-1\right)\left\|\sqrt{\varepsilon} \mathbf{E}^{n+1}\right\|_{L^{2}}^{2}-\left(\mathrm{e}^{2 \tau \eta}-1\right)\left\|\sqrt{\eta} \Phi^{n+1}\right\|_{L^{2}}^{2}-\sum_{i=1}^{3}\left(\frac{\tau^{3}}{2}\left\|D_{i}\left(\begin{array}{c}
\mathbf{E}^{n, 5+3 i} \\
\mathbf{H}^{n, 5+3 i} \\
\Phi^{n, 5+3 i}
\end{array}\right)\right\|^{2}+\frac{\tau^{6}}{16}\left\|D_{i}^{2}\left(\begin{array}{c}
\check{\mathbf{E}}^{n, 4+3 i} \\
\check{\mathbf{H}}^{n, 4+3 i} \\
\check{\Phi}^{n, 4+3 i}
\end{array}\right)\right\|^{2}\right) \\
&-\frac{\tau^{3}}{2}\left(\left\|B\left(\begin{array}{c}
\mathbf{E}^{n, 5} \\
\mathbf{H}^{n, 5} \\
\Phi^{n, 5}
\end{array}\right)\right\|^{2}+\left\|A\left(\begin{array}{c}
\mathbf{E}^{n, 2} \\
\mathbf{H}^{n, 2} \\
\Phi^{n, 2}
\end{array}\right)\right\|^{2}\right)-\frac{\tau^{6}}{16}\left(\left\|B^{2}\left(\begin{array}{c}
\check{\mathbf{E}}^{n, 4} \\
\check{\mathbf{H}}^{n, 4} \\
\check{\Phi}^{n, 4}
\end{array}\right)\right\|^{2}+\| \begin{array}{c}
\check{\mathbf{E}}^{n, 1} \\
\left.A^{2}\left(\begin{array}{c}
\check{\mathbf{H}}^{n, 1} \\
\check{\Phi}^{n, 1}
\end{array}\right) \|^{2}\right) .
\end{array}\right) .
\end{aligned}
$$

In consideration of the structural similarity between the expressions on the right hand side of (5.3) and (4.13), we choose for both schemes (3.17) and (3.18) the same initial data $\left(\mathbf{E}^{0}, \mathbf{H}^{0}, \Phi^{0}\right) \in Y$. The triangle inequality and (4.13) yield

$$
\begin{aligned}
& \int_{Q}\left(\mu\left|\mathbf{H}^{0}\right|^{2}+\varepsilon\left|\mathbf{E}^{0}\right|^{2}+\mu\left|\Phi^{0}\right|^{2}\right) \mathrm{d} x \\
& \leq 2 C_{o} \tau \sum_{k=1}^{N}\left(\left\|\mathbf{E}^{k}\right\|_{\mathrm{L}^{2}}^{2}+\left\|\Phi^{k}\right\|_{\mathrm{L}^{2}}^{2}+\left\|\mathbf{E}^{k}-\mathbf{E}_{c}^{k}\right\|_{\mathrm{L}^{2}}^{2}+\left\|\Phi^{k}-\Phi_{c}^{k}\right\|_{\mathrm{L}^{2}}^{2}\right) \\
& \left.\left.\quad+2 C_{o} \tau^{3} \sum_{k=0}^{N-1}\left(\left\|A\left(\begin{array}{c}
\mathbf{E}^{k, 2} \\
\mathbf{H}^{k, 2} \\
\Phi^{k, 2}
\end{array}\right)\right\|^{2}+\left\|A\left(\begin{array}{c}
\mathbf{E}^{k, 2}-\mathbf{E}_{c}^{k, 1} \\
\mathbf{H}^{k, 2}-\mathbf{H}_{c}^{k, 1} \\
\Phi^{k, 2}-\Phi_{c}^{k, 1}
\end{array}\right)\right\|^{2}+\| \begin{array}{c}
\mathbf{E}^{k, 5} \\
\mathbf{H}^{k, 5} \\
\Phi^{k, 5}
\end{array}\right)\left\|^{2}+\right\| \begin{array}{c}
\mathbf{E}^{k, 5}-\mathbf{E}_{c}^{k, 3} \\
\mathbf{H}^{k, 5}-\mathbf{H}_{c}^{k, 3} \\
\Phi^{k, 5}-\Phi_{c}^{k, 3}
\end{array}\right) \|^{2}\right) \\
& \left.\left.\quad+2 C_{o} \tau^{3} \sum_{k=0}^{N-1} \sum_{i=1}^{3}\left(\| \begin{array}{c}
\mathbf{E}^{k, 5+3 i} \\
\mathbf{H}_{i}^{k, 5+3 i} \\
\Phi^{k, 5+3 i}
\end{array}\right)\left\|^{2}\right\| \begin{array}{c}
\mathbf{E}^{k, 5+3 i}-\mathbf{E}_{c}^{k, 3+2 i} \\
\mathbf{H}^{k, 5+3 i}-\mathbf{H}_{c}^{k, 3+2 i} \\
\Phi^{k, 5+3 i}-\Phi_{c}^{k, 3+2 i}
\end{array}\right) \|^{2}\right),
\end{aligned}
$$

where $N=\max \{k \in \mathbb{N} \mid N \tau \leq 9 \stackrel{\circ}{\tau}$. The goal of the following subsection is to control the arising difference expressions by means of terms in $(5.3)$.

\subsection{Comparison of the damped and undamped schemes}

We first denote the differences between the iterates of (3.17) and (3.18) by

$$
\left(\begin{array}{l}
\mathbf{E}^{k, \Delta} \\
\mathbf{H}^{k, \Delta} \\
\Phi^{k, \Delta}
\end{array}\right):=\left(\begin{array}{c}
\mathbf{E}^{k}-\mathbf{E}_{c}^{k} \\
\mathbf{H}^{k}-\mathbf{H}_{c}^{k} \\
\Phi^{k}-\Phi_{c}^{k}
\end{array}\right)
$$

for $k \in \mathbb{N}$. The next lemma deals with the difference terms in (5.4) involving only substeps of both schemes. These can essentially be estimated by means of summands arising on the right hand side of (5.3), and the energy of the vectors $\left(\mathbf{E}^{k, \Delta}, \mathbf{H}^{k, \Delta}, \Phi^{k, \Delta}\right)$. Recall for the statement Definition (5.2). We denote $D_{-1}:=A$ and $D_{0}:=B$ to obtain a compact representation.

Lemma 5.1. Let $k \in \mathbb{N}_{0}, i \in\{0, \ldots, 3\}$, and $\tau \in(0, \stackrel{\circ}{\tau}]$. The estimates

$$
\left\|\left(I+\frac{\tau}{2} A\right)\left(\begin{array}{c}
\boldsymbol{E}^{k, 2}-\boldsymbol{E}_{c}^{k, 1} \\
\boldsymbol{H}^{k, 2}-\boldsymbol{H}_{c}^{k, 1} \\
\Phi^{k, 2}-\Phi_{c}^{k, 1}
\end{array}\right)\right\|^{2} \leq \frac{\tau^{6}}{16}\left\|A^{2}\left(\begin{array}{c}
\check{\boldsymbol{E}}^{k, 1} \\
\check{\boldsymbol{H}}^{k, 1} \\
\check{\Phi}^{k, 1}
\end{array}\right)\right\|^{2}+\tau^{3}\left\|A\left(\begin{array}{c}
\boldsymbol{E}^{k, 2} \\
\boldsymbol{H}^{k, 2} \\
\Phi^{k, 2}
\end{array}\right)\right\|^{2}+(1+\tau)\left\|\left(\begin{array}{c}
\boldsymbol{E}^{k, \Delta} \\
\boldsymbol{H}^{k, \Delta} \\
\Phi^{k, \Delta}
\end{array}\right)\right\|^{2},
$$




$$
\begin{aligned}
\left\|\left(I+\frac{\tau}{2} D_{i}\right)\left(\begin{array}{c}
\boldsymbol{E}^{k, 5+3 i}-\boldsymbol{E}_{c}^{k, 3+2 i} \\
\boldsymbol{H}^{k, 5+3 i}-\boldsymbol{H}_{c}^{k, 3+2 i} \\
\Phi^{k, 5+3 i}-\Phi_{c}^{k, 3+2 i}
\end{array}\right)\right\|^{2} \leq & \frac{\tau^{6}}{16}\left\|D_{i}^{2}\left(\begin{array}{c}
\check{\boldsymbol{E}}^{k, 4+3 i} \\
\check{\boldsymbol{H}}^{k, 4+3 i} \\
\check{\Phi}^{k, 4+3 i}
\end{array}\right)\right\|^{2}+\tau^{3}\left\|D_{i}\left(\begin{array}{c}
\boldsymbol{E}^{k, 5+3 i} \\
\boldsymbol{H}^{k, 5+3 i} \\
\Phi^{k, 5+3 i}
\end{array}\right)\right\|^{2} \\
& +(1+\tau)\left\|\left(I+\frac{\tau}{2} D_{i-1}\right)\left(\begin{array}{c}
\boldsymbol{E}^{k, 2+3 i}-\boldsymbol{E}_{c}^{k, 1+2 i} \\
\boldsymbol{H}^{k, 2+3 i}-\boldsymbol{H}_{c}^{k, 1+2 i} \\
\Phi^{k, 2+3 i}-\Phi_{c}^{k, 1+2 i}
\end{array}\right)\right\|^{2}
\end{aligned}
$$

are valid.

Proof. Since both inequalities can be proved in essentially the same way, we show only the first one. Definitions (5.1) and (4.1) on the one hand lead to the formula

$$
\left(\begin{array}{c}
\mathbf{E}^{k, 2}-\mathbf{E}_{c}^{k, 1} \\
\mathbf{H}^{k, 2}-\mathbf{H}_{c}^{k, 1} \\
\Phi^{k, 2}-\Phi_{c}^{k, 1}
\end{array}\right)=\left(I-\frac{\tau}{2} A\right)^{-1} \frac{\tau^{3}}{4} A^{2}\left(I-\frac{\tau^{2}}{4} A^{2}\right)^{-1}\left(\begin{array}{c}
\mathbf{E}^{k, 1} \\
\mathbf{H}^{k, 1} \\
\Phi^{k, 1}
\end{array}\right)+\left(I-\frac{\tau}{2} A\right)^{-1}\left(\begin{array}{c}
\mathbf{E}^{k, \Delta} \\
\mathbf{H}^{k, \Delta} \\
\Phi^{k, \Delta}
\end{array}\right) .
$$

On the other hand, Lemma 3.7 implies the identity

$$
\left(\frac{\tau^{3}}{4} A^{2}\left(I-\frac{\tau^{2}}{4} A^{2}\right)^{-1}\left(\begin{array}{c}
\mathbf{E}^{k, 1} \\
\mathbf{H}^{k, 1} \\
\Phi^{k, 1}
\end{array}\right),\left(\begin{array}{c}
\mathbf{E}^{k, \Delta} \\
\mathbf{H}^{k, \Delta} \\
\Phi^{k, \Delta}
\end{array}\right)\right)=-\left(\tau^{3 / 2} A\left(I-\frac{\tau}{2} A\right)^{-1}\left(\begin{array}{c}
\mathbf{E}^{k, 1} \\
\mathbf{H}^{k, 1} \\
\Phi^{k, 1}
\end{array}\right), \frac{\tau^{3 / 2}}{4} A\left(I-\frac{\tau}{2} A\right)^{-1}\left(\begin{array}{c}
\mathbf{E}^{k, \Delta} \\
\mathbf{H}^{k, \Delta} \\
\Phi^{k, \Delta}
\end{array}\right)\right) .
$$

Applying now $\left(I+\frac{\tau}{2} A\right)$ to (5.7), Lemma 3.7 and (5.2) yield the inequality

$$
\begin{aligned}
\left\|\left(I+\frac{\tau}{2} A\right)\left(\begin{array}{c}
\mathbf{E}^{k, 2}-\mathbf{E}_{c}^{k, 1} \\
\mathbf{H}^{k, 2}-\mathbf{H}_{c}^{k, 1} \\
\Phi^{k, 2}-\Phi_{c}^{k, 1}
\end{array}\right)\right\|^{2} \leq & \frac{\tau^{6}}{16}\left\|A^{2}\left(\begin{array}{c}
\check{\mathbf{E}}^{k, 1} \\
\check{\mathbf{H}}^{k, 1} \\
\check{\Phi}^{k, 1}
\end{array}\right)\right\|^{2}+\tau^{3}\left\|A\left(\begin{array}{c}
\mathbf{E}^{k, 2} \\
\mathbf{H}^{k, 2} \\
\Phi^{k, 2}
\end{array}\right)\right\|^{2}+\frac{\tau}{16}\left\|\tau A\left(I-\frac{\tau}{2} A\right)^{-1}\left(\begin{array}{c}
\mathbf{E}^{k, \Delta} \\
\mathbf{H}^{k, \Delta} \\
\Phi^{k, \Delta}
\end{array}\right)\right\|^{2} \\
& +\left\|\left(\begin{array}{c}
\mathbf{E}^{k, \Delta} \\
\mathbf{H}^{k, \Delta} \\
\Phi^{k, \Delta}
\end{array}\right)\right\|^{2}
\end{aligned}
$$

The first asserted estimate now follows from the identity $\tau A\left(I-\frac{\tau}{2} A\right)^{-1}=2\left(\left(I-\frac{\tau}{2} A\right)^{-1}-I\right)$.

It will be useful to have a slightly weaker version of the inequalities stated in Lemma 5.1.

Remark 5.2. Employing Lemma 3.7, we can weaken the first relation in Lemma 5.1, obtaining the estimate

$$
\frac{\tau^{2}}{4}\left\|A\left(\begin{array}{c}
\mathbf{E}^{k, 2}-\mathbf{E}_{c}^{k, 1} \\
\mathbf{H}^{k, 2}-\mathbf{H}_{c}^{k, 1} \\
\Phi^{k, 2}-\Phi_{c}^{k, 1}
\end{array}\right)\right\|^{2} \leq \frac{\tau^{6}}{16}\left\|A^{2}\left(\begin{array}{c}
\check{\mathbf{E}}^{k, 1} \\
\check{\mathbf{H}}^{k, 1} \\
0
\end{array}\right)\right\|^{2}+\tau^{3}\left\|A\left(\begin{array}{c}
\mathbf{E}^{k, 2} \\
\mathbf{H}^{k, 2} \\
\Phi^{k, 2}
\end{array}\right)\right\|^{2}+(1+\tau)\left\|\left(\begin{array}{c}
\mathbf{E}^{k, \Delta} \\
\mathbf{H}^{k, \Delta} \\
\Phi^{k, \Delta}
\end{array}\right)\right\|^{2} .
$$

Analogous modifications are also valid for the remaining estimates in Lemma 5.1.

Lemma 5.1 now leads to a bound for the energy of the differences $\left(\mathbf{E}^{k, \Delta}, \mathbf{H}^{k, \Delta}, \Phi^{k, \Delta}\right)$ by a employing a discrete integral over (5.3).

Lemma 5.3. The estimate

$$
\left\|\left(\begin{array}{l}
\boldsymbol{E}^{k+1, \Delta} \\
\boldsymbol{H}^{k+1, \Delta} \\
\Phi^{k+1, \Delta}
\end{array}\right)\right\|^{2} \leq C_{\Delta} \mathrm{e}^{6(k+1) \tau}\left(\left\|\left(\begin{array}{c}
\boldsymbol{E}^{0} \\
\boldsymbol{H}^{0} \\
\Phi^{0}
\end{array}\right)\right\|^{2}-\left\|\left(\begin{array}{c}
\boldsymbol{E}^{k+1} \\
\boldsymbol{H}^{k+1} \\
\Phi^{k+1}
\end{array}\right)\right\|^{2}\right)
$$

is true with a constant $C_{\Delta}=C_{\Delta}(\varepsilon, \mu, \sigma, \eta)>0$ for all $-1 \leq k \leq N-1$ and $\tau \in(0, \stackrel{\circ}{\tau}]$. 
Proof. Since both schemes start from the same initial field, the estimate is clear for $k=-1$. So we assume $k \geq 0$. Similar to the proof of Lemma 5.1, (4.1) and (5.1) yield the representation

$$
\begin{aligned}
\left(\begin{array}{l}
\mathbf{E}^{k+1, \Delta} \\
\mathbf{H}^{k+1, \Delta} \\
\Phi^{k+1, \Delta}
\end{array}\right) & =\left(\begin{array}{ccc}
\mathrm{e}^{-\tau \sigma}-1 & 0 & 0 \\
0 & 0 & 0 \\
0 & 0 & \mathrm{e}^{-\tau \eta}-1
\end{array}\right)\left(I+\frac{\tau}{2} D_{3}\right)\left(\begin{array}{l}
\mathbf{E}^{k, 14} \\
\mathbf{H}^{k, 14} \\
\Phi^{k, 14}
\end{array}\right)+\left(I+\frac{\tau}{2} D_{3}\right)\left(\begin{array}{c}
\mathbf{E}^{k, 14}-\mathbf{E}_{c}^{k, 9} \\
\mathbf{H}^{k, 14}-\mathbf{H}_{c}^{k, 9} \\
\Phi^{k, 14}-\Phi_{c}^{k, 9}
\end{array}\right) \\
& =\left(\begin{array}{ccc}
\mathrm{e}^{-\tau \sigma}-1 & 0 & 0 \\
0 & 0 & 0 \\
0 & 0 & \mathrm{e}^{-\tau \eta}-1
\end{array}\right)\left(\begin{array}{ccc}
\mathrm{e}^{\tau \sigma} & 0 & 0 \\
0 & I & 0 \\
0 & 0 & \mathrm{e}^{\tau \eta}
\end{array}\right)\left(\begin{array}{c}
\mathbf{E}^{k+1} \\
\mathbf{H}^{k+1} \\
\Phi^{k+1}
\end{array}\right)+\left(I+\frac{\tau}{2} D_{3}\right)\left(\begin{array}{c}
\mathbf{E}_{c}^{k, 9} \\
\mathbf{H}^{k, 14}-\mathbf{H}_{c}^{k, 9} \\
\Phi^{k, 14}-\Phi_{c}^{k, 9}
\end{array}\right)
\end{aligned}
$$

Set $C_{\sigma \eta}:=\max \left\{\|\sigma\|_{\infty},\|\eta\|_{\infty}\right\}$. In view of the inequality $\left\|\mathrm{e}^{-\tau \xi}-1\right\|_{\infty} \leq \tau\|\xi\|_{\infty}$ for $\xi \in\{\sigma, \eta\}$, we thus infer the relations

$$
\left\|\left(\begin{array}{l}
\mathbf{E}^{k+1, \Delta} \\
\mathbf{H}^{k+1, \Delta} \\
\Phi^{k+1, \Delta}
\end{array}\right)\right\|^{2} \leq 2 \tau C_{\sigma \eta}^{2} \mathrm{e}^{2 C_{\sigma \eta}}\left\|\left(\begin{array}{c}
\mathbf{E}^{k+1} \\
0 \\
\Phi^{k+1}
\end{array}\right)\right\|^{2}+(1+\tau)\left\|\left(I+\frac{\tau}{2} D_{3}\right)\left(\begin{array}{c}
\mathbf{E}^{k, 14}-\mathbf{E}_{c}^{k, 9} \\
\mathbf{H}^{k, 14}-\mathbf{H}_{c}^{k, 9} \\
\Phi^{k, 14}-\Phi_{c}^{k, 9}
\end{array}\right)\right\|^{2} .
$$

Lemma 5.1, (5.3), and the assumption $\tau \leq 1$ now imply the estimates

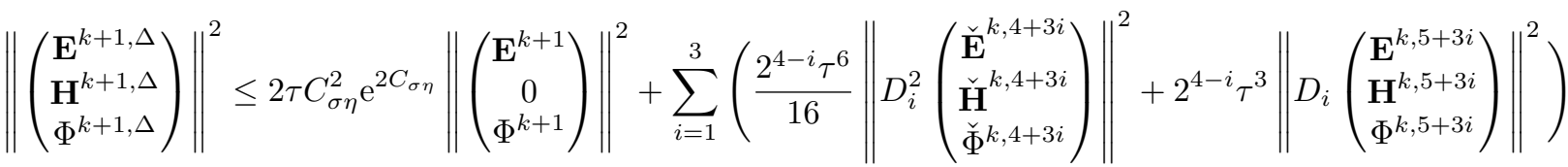

$$
\begin{aligned}
& +\tau^{6}\left\|B^{2}\left(\begin{array}{c}
\check{\mathbf{E}}^{k, 4} \\
\check{\mathbf{H}}^{k, 4} \\
\check{\Phi}^{k, 4}
\end{array}\right)\right\|^{2}+16 \tau^{3}\left\|B\left(\begin{array}{c}
\mathbf{E}^{k, 5} \\
\mathbf{H}^{k, 5} \\
\Phi^{k, 5}
\end{array}\right)\right\|^{2}+2 \tau^{6}\left\|A^{2}\left(\begin{array}{c}
\check{\mathbf{E}}^{k, 1} \\
\check{\mathbf{H}}^{k, 1} \\
\check{\Phi}^{k, 1}
\end{array}\right)\right\|^{2} \\
& +32 \tau^{3}\left\|A\left(\begin{array}{c}
\mathbf{E}^{k, 2} \\
\mathbf{H}^{k, 2} \\
\Phi^{k, 2}
\end{array}\right)\right\|^{2}+(1+\tau)^{6}\left\|\left(\begin{array}{c}
\mathbf{E}^{k, \Delta} \\
\mathbf{H}^{k, \Delta} \\
\Phi^{k, \Delta}
\end{array}\right)\right\|^{2} \\
& \leq C_{\Delta}\left(\left\|\left(\begin{array}{c}
\mathbf{E}^{k} \\
\mathbf{H}^{k} \\
\Phi^{k}
\end{array}\right)\right\|^{2}-\left\|\left(\begin{array}{c}
\mathbf{E}^{k+1} \\
\mathbf{H}^{k+1} \\
\Phi^{k+1}
\end{array}\right)\right\|^{2}\right)+\mathrm{e}^{6 \tau}\left\|\left(\begin{array}{c}
\mathbf{E}^{k, \Delta} \\
\mathbf{H}^{k, \Delta} \\
\Phi^{k, \Delta}
\end{array}\right)\right\|^{2}
\end{aligned}
$$

with a constant $C_{\Delta}=C_{\Delta}(\varepsilon, \mu, \sigma, \eta)>0$ being independent of $k$ and $\tau$. In presence of the initial choice $\left(\mathbf{E}^{0, \Delta}, \mathbf{H}^{0, \Delta}, \Phi^{0, \Delta}\right)=0$, we conclude by induction the relation

$$
\left\|\left(\begin{array}{c}
\mathbf{E}^{k+1, \Delta} \\
\mathbf{H}^{k+1, \Delta} \\
\Phi^{k+1, \Delta}
\end{array}\right)\right\|^{2} \leq C_{\Delta} \mathrm{e}^{6(k+1) \tau} \sum_{j=0}^{k}\left(\left\|\left(\begin{array}{c}
\mathbf{E}^{j} \\
\mathbf{H}^{j} \\
\Phi^{j}
\end{array}\right)\right\|^{2}-\left\|\left(\begin{array}{c}
\mathbf{E}^{j+1} \\
\mathbf{H}^{j+1} \\
\Phi^{j+1}
\end{array}\right)\right\|^{2}\right),
$$

yielding the assertion.

\subsection{Demonstration of the exponential stability for the damped scheme}

The observability estimate from Theorem 4.2, the energy identity (5.3) and the estimates from Lemmas 5.1 and 5.3 at hand, we are now in the position to conclude Theorem 3.9.

Proof of Theorem 3.9. Let $\stackrel{\circ}{\tau}=\zeta \max \left\{\frac{1}{2}, \frac{\sqrt{2}}{\kappa_{Y}}\right\}$, and $N:=\max \left\{k \in \mathbb{N} \mid k \tau \leq 9 \stackrel{\circ}{\tau}\right.$. The initial data $\left(\mathbf{E}^{0}, \mathbf{H}^{0}, \Phi^{0}\right)$ for scheme (3.18) is for the time being assumed to belong to $Y$. The proof mainly consists in estimating all terms on the right hand side of (5.4). The difference expressions are first treated, and it suffices to consider only the last two summands, since all others can be handled with similar arguments. In the following, $\tilde{C}>0$ denotes a constant that is allowed to change from line to line, but depends solely on $\varepsilon, \mu, \sigma, \eta$ and $Q$. We first weaken (5.6) for $i=3$ in 
the spirit of Remark 5.2, and insert all other estimates from Lemma 5.1 recursively into each other. Since $\tau<1$, we obtain the relations

$$
\begin{gathered}
\tau^{3}\left\|D_{3}\left(\begin{array}{c}
\mathbf{E}^{k, 14}-\mathbf{E}_{c}^{k, 9} \\
\mathbf{H}^{k, 14}-\mathbf{H}_{c}^{k, 9} \\
\Phi^{k, 14}-\Phi_{c}^{k, 9}
\end{array}\right)\right\|^{2} \leq \tilde{C}\left(\tau^{7}\left\|D_{3}^{2}\left(\begin{array}{c}
\check{\mathbf{E}}^{k, 13} \\
\check{\mathbf{H}} \\
\check{\Phi}^{k, 13}
\end{array}\right)\right\|^{2}+\tau^{4}\left\|D_{3}\left(\begin{array}{c}
\mathbf{E}^{k, 14} \\
\mathbf{H}^{k, 14} \\
\Phi^{k, 14}
\end{array}\right)\right\|^{2}+\tau\left\|\left(I+\frac{\tau}{2} D_{2}\right)\left(\begin{array}{c}
\mathbf{E}^{k, 11}-\mathbf{E}_{c}^{k, 7} \\
\mathbf{H}^{k, 11}-\mathbf{H}_{c}^{k, 7} \\
\Phi^{k, 11}-\Phi_{c}^{k, 7}
\end{array}\right)\right\|^{2}\right) \\
\leq \tilde{C}\left[\sum_{i=1}^{3}\left(\tau^{7}\left\|D_{i}^{2}\left(\begin{array}{c}
\check{\mathbf{E}}^{k, 4+3 i} \\
\check{\mathbf{H}}^{k, 4+3 i} \\
\check{\Phi}^{k, 4+3 i}
\end{array}\right)\right\|^{2}+\tau^{4}\left\|D_{i}\left(\begin{array}{c}
\mathbf{E}^{k, 5+3 i} \\
\mathbf{H}^{k, 5+3 i} \\
\Phi^{k, 5+3 i}
\end{array}\right)\right\|^{2}\right)+\tau^{7}\left\|B^{2}\left(\begin{array}{c}
\check{\mathbf{E}}^{k, 4} \\
\check{\mathbf{H}}^{k, 4} \\
\check{\Phi}^{k, 4}
\end{array}\right)\right\|^{2}+\tau^{4}\left\|B\left(\begin{array}{c}
\mathbf{E}^{k, 5} \\
\mathbf{H}^{k, 5} \\
\Phi^{k, 5}
\end{array}\right)\right\|^{2}\right. \\
\left.+\tau^{7}\left\|A^{2}\left(\begin{array}{c}
\check{\mathbf{E}}^{k, 1} \\
\check{\mathbf{H}}^{k, 1} \\
\check{\Phi}^{k, 1}
\end{array}\right)\right\|^{2}+\tau^{4}\left\|A\left(\begin{array}{c}
\mathbf{E}^{k, 2} \\
\mathbf{H}^{k, 2} \\
\Phi^{k, 2}
\end{array}\right)\right\|^{2}+\tau\left\|\left(\begin{array}{c}
\mathbf{E}^{k, \Delta} \\
\mathbf{H}^{k, \Delta} \\
\Phi^{k, \Delta}
\end{array}\right)\right\|^{2}\right] .
\end{gathered}
$$

Similar reasoning shows that all other difference terms on the right hand side of (5.4) are bounded from above by the right hand side of (5.8) if the number $\tilde{C}$ is appropriately modified. As a result, we infer in view of (5.4), (5.3), the relation $N \tau \leq 9 \stackrel{\circ}{\tau}$, and Lemma 5.3 the inequalities

$$
\begin{aligned}
\left\|\left(\begin{array}{c}
\mathbf{E}^{0} \\
\mathbf{H}^{0} \\
\Phi^{0}
\end{array}\right)\right\|^{2} & \leq \tilde{C} \sum_{k=0}^{N-1}\left(\left\|\left(\begin{array}{c}
\mathbf{E}^{k} \\
\mathbf{H}^{k} \\
\Phi^{k}
\end{array}\right)\right\|^{2}-\left\|\left(\begin{array}{c}
\mathbf{E}^{k+1} \\
\mathbf{H}^{k+1} \\
\Phi^{k+1}
\end{array}\right)\right\|^{2}\right)+\tilde{C} \tau \mathrm{e}^{54 \tau} \sum_{k=1}^{N-1}\left(\left\|\left(\begin{array}{c}
\mathbf{E}^{0} \\
\mathbf{H}^{0} \\
\Phi^{0}
\end{array}\right)\right\|^{2}-\left\|\left(\begin{array}{c}
\mathbf{E}^{k+1} \\
\mathbf{H}^{k+1} \\
\Phi^{k+1}
\end{array}\right)\right\|^{2}\right) \\
& \leq \tilde{C} \tau \mathrm{e}^{54 \tau}\left(\left\|\left(\begin{array}{c}
\mathbf{E}^{0} \\
\mathbf{H}^{0} \\
\Phi^{0}
\end{array}\right)\right\|^{2}-\left\|\left(\begin{array}{c}
\mathbf{E}^{N} \\
\mathbf{H}^{N} \\
\Phi^{N}
\end{array}\right)\right\|^{2}\right),
\end{aligned}
$$

where the last estimate is equivalent to

$$
\left\|\left(\begin{array}{c}
\mathbf{E}^{N} \\
\mathbf{H}^{N} \\
\Phi^{N}
\end{array}\right)\right\|^{2} \leq\left(1-\frac{1}{\tilde{C} \dot{\tau} \mathrm{e}^{54 \tau}}\right)\left\|\left(\begin{array}{c}
\mathbf{E}^{0} \\
\mathbf{H}^{0} \\
\Phi^{0}
\end{array}\right)\right\|^{2} .
$$

In view of Lemmas 3.11 and 3.16, Corollary 3.15, and the assumptions (1.2) and (3.2), we can iterate the entire argument, and infer with the same constant $\tilde{C}$ as in (5.9) the relations

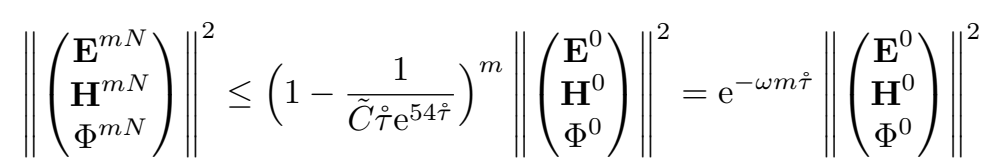

with $\omega:=\frac{1}{\tau} \ln \left(\frac{\tilde{C} \tau \mathrm{e}^{54 \dot{\tau}}}{\tilde{C} \tau \mathrm{e}^{54 \tau}-1}\right)>0$ for all $m \in \mathbb{N}$. The asserted exponential decay for starting values in $Y$ can now be concluded by means of standard arguments. The same decay rate, however, is also true for initial data in $X=\mathrm{L}^{2}(Q)^{7}$, employing the density of $Y$ in $X$.

\section{ERROR ANALYSIS}

The purpose of this section is to establish a rigorous error result in the dual space $Y^{*}$ of $Y$ for scheme (3.18) in the abstract time-discrete setting. We therefore show the stability of (3.18) in $Y$, and employ the regularity statements derived in Section 3. The final error result is then deduced by estimating the local error in Lemma 6.4 and controlling the error propagation in the proof of Theorem 6.5. 


\subsection{Stability of the damped scheme}

Let $L \in\left\{A_{Y}, B_{Y}, D_{1, Y}, D_{2, Y}, D_{3, Y}\right\}$. Lemma 3.11 and Corollary 3.15 estimate the Cayley-Transform by

$$
\left\|S_{\tau}(L)\right\|_{\mathscr{B}(Y)} \leq \mathrm{e}^{3 \kappa_{Y} \tau}, \quad \tau \in\left(0, \tau_{0}\right],
$$

where $\tau_{0}>0$ is a constant depending only on $\kappa_{Y}$. In view of (1.2) and (3.2), the operator associated to the last substep satisfies the relation

$$
\left\|\left(\begin{array}{ccc}
\mathrm{e}^{-\tau \sigma} & 0 & 0 \\
0 & I & 0 \\
0 & 0 & \mathrm{e}^{-\tau \eta}
\end{array}\right)\right\|_{\mathscr{B}(Y)} \leq \mathrm{e}^{\tau \tilde{C}_{S}}, \quad \tau \geq 0
$$

with a uniform constant $\tilde{C}_{S}=\tilde{C}_{S}(\sigma, \eta)>0$. The next lemma also bounds the operator $V_{\tau}(L)$ from $(3.16)$ in $Y$. Additional estimates are moreover given which prove to be useful when deriving bounds for the local error.

Lemma 6.1. Let $L \in\left\{A_{Y}, B_{Y}, D_{1, Y}, D_{2, Y}, D_{3, Y}\right\}$. The operator $V_{\tau}(L)$ is well-defined in $Y$ for all $\tau \in\left(0, \frac{1}{\kappa_{Y}}\right)$. There moreover is a constant $\tilde{\tau}_{0} \in\left(0, \min \left\{\frac{1}{6}, \frac{1}{\kappa_{Y}}\right\}\right)$ with

$$
\left\|\frac{\tau^{2}}{4} L^{2}\left(I-\frac{\tau^{2}}{4} L^{2}\right)^{-1}\right\|_{\mathscr{B}(Y)} \leq 3, \quad\left\|V_{\tau}(L)\right\|_{\mathscr{B}(Y)} \leq \frac{1}{1-3 \tau}
$$

for all $\tau \in\left(0, \tilde{\tau}_{0}\right)$.

Proof. Lemma 3.11 and Corollary 3.15 yield that the mapping $\left(I-\tau^{2} L^{2}\right)^{-1}$ is bounded on $Y$, and satisfies the inequality

$$
\left\|\left(I-\tau^{2} L^{2}\right)^{-1}\right\|_{\mathscr{B}(Y)} \leq \frac{1}{\left(1-\tau \kappa_{Y}\right)^{2}}
$$

for $\tau \in\left(0, \frac{1}{\kappa_{Y}}\right)$. There in particular is a number $\tilde{\tau}_{0} \in\left(0, \min \left\{\frac{1}{6}, \frac{1}{\kappa_{Y}}\right\}\right)$, depending only on $\kappa_{Y}$, such that the estimate $\left\|\left(I-\frac{\tau^{2}}{4} L^{2}\right)^{-1}\right\|_{\mathscr{B}(Y)} \leq 2$ is valid for $\tau \in\left(0, \tilde{\tau}_{0}\right)$. The formula $\frac{\tau^{2}}{4} L^{2}\left(I-\frac{\tau^{2}}{4} L^{2}\right)^{-1}=-I+\left(I-\frac{\tau^{2}}{4} L^{2}\right)^{-1}$ further yields $\left\|\frac{\tau^{2}}{4} L^{2}\left(I-\frac{\tau^{2}}{4} L^{2}\right)^{-1}\right\|_{\mathscr{B}(Y)} \leq 3$. Since $\tilde{\tau}_{0}<\frac{1}{6}$, the operator $V_{\tau}(L)$ is consequently well-defined and bounded on $Y$, satisfying the relations

$$
\left\|V_{\tau}(L)\right\|_{\mathscr{B}(Y)}=\left\|\left(I-\frac{\tau^{3}}{4} L^{2}\left(I-\frac{\tau^{2}}{4} L^{2}\right)^{-1}\right)^{-1}\right\|_{\mathscr{B}(Y)} \leq \frac{1}{1-\tau\left\|\frac{\tau^{2}}{4} L^{2}\left(I-\frac{\tau^{2}}{4} L^{2}\right)^{-1}\right\|_{\mathscr{B}(Y)}} \leq \frac{1}{1-3 \tau}
$$

for all $\tau \in\left(0, \tilde{\tau}_{0}\right)$.

We conclude this subsection with the unconditional stability of (3.18) in $Y$.

Proposition 6.2. Let $\varepsilon, \mu, \sigma, \eta$ satisfy (1.2) and (3.2), and let $T>0$. There are constants $C_{\text {stab }}, \check{\tau}_{0}>0$ with

$$
\left\|\left(\begin{array}{c}
\boldsymbol{E}^{n} \\
\boldsymbol{H}^{n} \\
\Phi^{n}
\end{array}\right)\right\|_{Y} \leq \mathrm{e}^{C_{\text {stab }} T}\left\|\left(\begin{array}{c}
\boldsymbol{E}^{0} \\
\boldsymbol{H}^{0} \\
\Phi^{0}
\end{array}\right)\right\|_{Y}
$$

for all $\left(\boldsymbol{E}^{0}, \boldsymbol{H}^{0}, \Phi^{0}\right) \in Y, \tau \in\left(0, \check{\tau}_{0}\right)$ and $n \in \mathbb{N}$ with $n \tau \leq T$. Here, $C_{\text {stab }}, \check{\tau}_{0}>0$ depend only on $\varepsilon, \mu, \sigma, \eta$ and $Q$.

Proof. Set $\check{\tau}_{0}:=\min \left\{\tau_{0}, \tilde{\tau}_{0}\right\} \in\left(0, \frac{1}{6}\right)$ with $\tau_{0}$ from Lemma 3.11 and Corollary 3.15 , and $\tilde{\tau}_{0}$ from Lemma 6.1. Denote also $N:=\max \{k \in \mathbb{N} \mid k \tau \leq T\}$. The estimates (6.1), (6.2), and Lemma 6.1 imply the bound

$$
\left\|\left(\begin{array}{c}
\mathbf{E}^{n} \\
\mathbf{H}^{n} \\
\Phi^{n}
\end{array}\right)\right\|_{Y} \leq\left(\mathrm{e}^{\tau \tilde{C}_{S}} \frac{1}{(1-3 \tau)^{5}} \mathrm{e}^{15 \kappa_{Y} \tau}\right)^{N}\left\|\left(\begin{array}{c}
\mathbf{E}^{0} \\
\mathbf{H}^{0} \\
\Phi^{0}
\end{array}\right)\right\|_{Y}, \quad \tau \in\left(0, \check{\tau}_{0}\right) .
$$

Choose $C=\frac{3}{1-3 \check{\tau}_{0}}>0$, then $\frac{1}{1-3 \tau} \leq \mathrm{e}^{C \tau}$ for $\tau \in\left(0, \check{\tau}_{0}\right)$. Since $N \tau \leq T$, the asserted estimate is valid for $C_{\text {stab }}:=\tilde{C}_{S}+5 C+15 \kappa_{Y}$. 


\subsection{Auxiliary constructions}

Take $\tau \in\left(0, \check{\tau}_{0}\right)$ with $\check{\tau}_{0}$ from Proposition 6.2. We first expand the substeps of the scheme (3.18) to obtain a convenient representation of the local error. Let $L \in\left\{A_{Y}, B_{Y}, D_{1, Y}, D_{2, Y}, D_{3, Y}\right\}$. Employing the supplementary operators

$$
V_{\tau}^{(i)}(L):=\sum_{n=i}^{\infty}\left(\frac{\tau^{3}}{4} L^{2}\left(I-\frac{\tau^{2}}{4} L^{2}\right)^{-1}\right)^{n}, \quad i \in\{1,2\},
$$

the operator $V_{\tau}(L)$ from (3.16) can be expressed via the formula

$$
V_{\tau}(L)=\sum_{n=0}^{\infty}\left(\frac{\tau^{3}}{4} L^{2}\left(I-\frac{\tau^{2}}{4} L^{2}\right)^{-1}\right)^{n}=I+V_{\tau}^{(1)}(L)=I+\frac{\tau^{3}}{4} L^{2}\left(I-\frac{\tau^{2}}{4} L^{2}\right)^{-1}+V_{\tau}^{(2)}(L) .
$$

Lemma 6.1 further yields the relations

$$
\left\|V_{\tau}^{(1)}(L)\right\|_{\mathscr{B}(Y)} \leq 6 \tau, \quad\left\|V_{\tau}^{(2)}(L)\right\|_{\mathscr{B}(Y)} \leq 18 \tau^{2} .
$$

By means of the mappings

$$
F^{V}(j, k, L):= \begin{cases}V_{\tau}(L) & \text { if } j=k=0, \\ V_{\tau}^{(k)}(L) & \text { if } j=k>0, \\ \frac{\tau^{3 j}}{4^{j}} L^{2 j}\left(I-\frac{\tau^{2}}{4} L^{2}\right)^{-j} & \text { if } j<k,\end{cases}
$$

for $j \leq k \in\{0,1,2\}$, we can rewrite (6.3) in the convenient way

$$
F^{V}(0,0, L)=\sum_{j=0}^{k} F^{V}(j, k, L), \quad k \in\{0,1,2\} .
$$

The Cayley-Transform $S_{\tau}(L)$ can be expanded in a similar way. Defining

$$
F(j, k, L):= \begin{cases}S_{\tau}(L) & \text { if } j=k=0, \\ \frac{\tau^{k}}{2^{k}}\left(I+S_{\tau}(L)\right) L^{k} & \text { if } j=k>0, \\ \frac{\tau^{j}}{j !} L^{j} & \text { if } j<k,\end{cases}
$$

for $j \leq k \in\{0,1,2\}$, the formula

$$
F(0,0, L)=\sum_{j=0}^{k} F(j, k, L)
$$

is valid on $\mathcal{D}\left(L^{k}\right)$ for $k \in\{0,1,2\}$, see Section 4.1 of [8]. To obtain also useful representations for the semigroup $\left(\mathrm{e}^{t M_{\text {ext }, 1}}\right)_{t \geq 0}$ and the last substep of (3.18), we make in the following use of auxiliary operators that have already been employed in $[17,18,9,10,8]$. Let $\tilde{L}$ be the generator of a strongly continuous semigroup $\left(\mathrm{e}^{t \tilde{L}}\right)_{t \geq 0}$ on $X_{1}$, and set

$$
\phi_{j}(\tilde{L}):=\int_{0}^{1} \frac{\zeta^{j-1}}{(j-1) !} \mathrm{e}^{(1-\zeta) \tilde{L}} \mathrm{~d} \zeta, \quad j \in \mathbb{N}, \quad \phi_{0}(\tilde{L}):=\mathrm{e}^{\tilde{L}} .
$$

Note that these operators are bounded on $X_{1}$, and that the vector $\phi_{j}(\tilde{L}) z$ belongs to $\mathcal{D}(\tilde{L})$ for $j \in \mathbb{N}, z \in X_{1}$. An integration by parts further yields the important recursion formula

$$
\tilde{L} \phi_{j+1}(\tilde{L})=\phi_{j}(\tilde{L})-\frac{1}{j !} I, \quad j \in \mathbb{N}_{0} .
$$

Choosing $\tilde{L}=\tau M_{\text {ext,1 }}$, Proposition 3.5 implies the estimate

$$
\left\|\phi_{j}\left(\tau M_{\mathrm{ext}, 1}\right)\right\|_{\mathscr{B}\left(X_{1}\right)} \leq \frac{2 C_{\mathrm{stab}, 1}}{j !} .
$$


Selecting

$$
\tilde{L}=K_{d}:=\left(\begin{array}{ccc}
-\sigma & 0 & 0 \\
0 & 0 & 0 \\
0 & 0 & -\eta
\end{array}\right)
$$

relation (6.7) leads to the identities

$$
\mathrm{e}^{\tau K_{d}}=I+\tau K_{d} \phi_{1}\left(\tau K_{d}\right)=I+\tau K_{d}+\tau^{2} K_{d}^{2} \phi_{2}\left(\tau K_{d}\right) .
$$

They are equivalent to the equation

$$
F^{\sigma}(0,0)=\sum_{j=0}^{k} F^{\sigma}(j, k), \quad k \in\{0,1,2\},
$$

with

$$
F^{\sigma}(j, k):= \begin{cases}\mathrm{e}^{\tau K_{d}} & \text { if } j=k=0 \\ \tau^{j} K_{d}^{j} \phi_{j}\left(\tau K_{d}\right) & \text { if } j=k>0 \\ \tau^{j} K_{d}^{j} & \text { if } j<k\end{cases}
$$

for $j \leq k \in\{0,1,2\}$.

\subsection{Convergence result for the damped ADI scheme}

To estimate the local error of (3.18) in $Y^{*}$, extrapolation of operators to $X$ is employed. We therefore collect associated facts in the following remark which will be employed in the subsequent proofs without further notice.

Remark 6.3. Let $L \in\left\{M_{\text {ext }}, A, B, D_{1}, D_{2}, D_{3}\right\}$, and denote the extrapolation space of $X$ with respect to $L$ by $X_{-1}^{L}$. Proposition 2.10 .2 in [31] yields $X_{-1}^{L} \cong \mathcal{D}\left(L^{*}\right)^{*}$, and the inclusion of $Y$ in $\mathcal{D}(L)=\mathcal{D}\left(L^{*}\right)$ by definition of $Y$ thus implies $X_{-1}^{L} \subseteq Y^{*}$. There is also a useful relation between the extrapolation operator $L_{-1}$ and the bidual operator $\left(L^{*}\right)^{*}$ of $L$. In view of the continuity of $\left(L^{*}\right)^{*}: X \rightarrow X_{-1}^{L}$ and the identity

$$
\left\langle\left(L^{*}\right)^{*} x, y\right\rangle_{Y^{*} \times Y}=(L x, y), \quad y \in Y, x \in \mathcal{D}(L),
$$

we infer that $\left(L^{*}\right)^{*}$ is the unique continuous extension of $L$ to $X$, see Proposition 2.10.3 in [31]. As a result, the crucial relations

$$
\left\langle L_{-1} x, y\right\rangle_{Y^{*} \times Y}=\left\langle\left(L^{*}\right)^{*} x, y\right\rangle_{Y^{*} \times Y}=\left(x, L^{*} y\right), \quad x \in X, y \in Y .
$$

are obtained. There is also need to extend some bounded operators from $X$ to $Y^{*}$. Let $P \in \mathscr{B}(X)$ with an adjoint operator $P^{*}$ leaving $Y$ invariant. By setting $\tilde{P}:=\left(\left.P^{*}\right|_{Y}\right)^{*}$, we obtain the unique continuous extension of $P$ to $Y^{*}$. This extension in particular satisfies the identity

$$
\langle\tilde{P} z, y\rangle_{Y^{*} \times Y}=\left\langle z, P^{*} y\right\rangle_{Y^{*} \times Y}, \quad z \in Y^{*}, y \in Y,
$$

see Proposition 2.9.3 in [31]. Lemmas 3.11 and 3.16, and Corollary 3.15 show that this extension procedure works for the considered Cayley-Transforms, the operators $F^{V}(j, k, L)$ and $F^{\sigma}(j, k)$ for $j \leq k \in\{1,2\}$.

We are now in the position to estimate the local error of (3.18) in $Y^{*}$ by adapting arguments from the proof of Theorem 4.1 in [8] to the current scheme. The solution of the continuous problem (3.1) at time $t \geq 0$ with initial datum $\left(\mathbf{E}_{0}, \mathbf{H}_{0}, \Phi_{0}\right)$ is in the following abbreviated by $v(t)$. The corresponding approximation at $n \tau$ by scheme (3.18) is denoted by $v^{n}$.

Lemma 6.4. Let $\varepsilon, \mu, \sigma, \eta$ satisfy (1.2) and (3.2). The local error satisfies the estimate

$$
\left|\left(v^{1}-v(\tau), y\right)\right| \leq C_{\text {loc }} \tau^{2}\|v(0)\|_{X_{1}}\|y\|_{Y}
$$

for all $y \in Y$, initial data $v(0)=v^{0} \in X_{1}$ and $\tau \in\left(0, \check{\tau}_{0}\right)$. $C_{\mathrm{loc}}$ and $\check{\tau}_{0}$ are two positive constants depending only on $\varepsilon, \mu, \sigma, \eta, Q$. 
Proof. Throughout the proof, the constant $C$ depends only on $\varepsilon, \mu, \sigma, \eta, Q$, and is allowed to change from line to line. We moreover employ (1.2) and (3.2) without further notice. Let $\check{\tau}_{0}$ be the constant from Proposition 6.2 , and denote by $S(\tau)$ the mapping that maps the input of (3.18) onto its output. Employing (6.7) for $\tilde{L}=\tau M_{\text {ext,1 }}$, the local error has the representation

$$
v^{1}-v(\tau)=\left(S(\tau)-\mathrm{e}^{\tau M_{\mathrm{ext}, 1}}\right) v^{0}=\left(S(\tau)-I-\tau M_{\mathrm{ext}}-\tau^{2} M_{\mathrm{ext}-1} M_{\mathrm{ext}, 1} \phi_{2}\left(\tau M_{\mathrm{ext}, 1}\right)\right) v^{0},
$$

where $M_{\text {ext-1 }}$ denotes the extrapolation of $M_{\text {ext }}$ to $X$. Estimate (6.8) then implies for the last term on the right hand side of (6.10) the relations

$$
\left|\left\langle\tau^{2} M_{\text {ext }-1} M_{\text {ext }, 1} \phi_{2}\left(\tau M_{\text {ext }, 1}\right) v^{0}, y\right\rangle_{Y^{*} \times Y}\right|=\left|\left(\tau^{2} M_{\text {ext }} \phi_{2}\left(\tau M_{\text {ext }, 1}\right) v^{0}, M_{\text {ext }}^{*} y\right)\right| \leq C \tau^{2}\left\|v^{0}\right\|_{X_{1}}\|y\|_{Y} .
$$

The remaining three summands on the right hand side of (6.10) are now expanded in terms of the supplementary operators from Subsection 6.2. Product signs thereby in the following mean that the respective operators are concatenated in such a way that the index decreases from left to right. On the one hand, we obtain the formula

$$
\begin{gathered}
v^{0}+\tau M_{\mathrm{ext}} v^{0}=\sum_{k=0}^{1} \sum_{j_{1}+\cdots+j_{6}=k} F^{\sigma}\left(j_{6}, 2-\sum_{i=1}^{5} j_{i}\right) \prod_{l=1}^{3}\left(F\left(j_{2+l}, 2-\sum_{i=1}^{l+1} j_{i}, D_{l}\right) F^{V}\left(0,2, D_{l}\right)\right) F\left(j_{2}, 2-j_{1}, B\right) \\
\cdot F^{V}(0,2, B) F\left(j_{1}, 2, A\right) F^{V}(0,2, A) v^{0}
\end{gathered}
$$

Plugging the representation formulas for the splitting steps from Subsection 6.2 into the definition of $S(\tau)$, we on the other hand derive the identities

$$
\begin{aligned}
S(\tau) v^{0}= & \sum_{j_{1}=0}^{2} \sum_{r_{1}=0}^{2} F^{\sigma}(0,0) \prod_{l=1}^{3}\left(F\left(0,0, D_{l}\right) F^{V}\left(0,0, D_{l}\right)\right) F(0,0, B) F^{V}(0,0, B) F\left(j_{1}, 2, A\right) F^{V}\left(r_{1}, 2, A\right) v^{0} \\
= & \sum_{j_{1}=0}^{2} \sum_{j_{2}=0}^{2-j_{1}} \sum_{r_{1}=0}^{2} \sum_{r_{2}=0}^{2-r_{1}} F^{\sigma}(0,0) \prod_{l=1}^{3}\left(F\left(0,0, D_{l}\right) F^{V}\left(0,0, D_{l}\right)\right) F\left(j_{2}, 2-j_{1}, B\right) F^{V}\left(r_{2}, 2-r_{1}, B\right) F\left(j_{1}, 2, A\right) \\
& \cdot F^{V}\left(r_{1}, 2, A\right) v^{0} \\
= & \sum_{k=0}^{2} \sum_{j_{1}+\cdots+j_{6}=k} \sum_{s=0}^{2} \sum_{r_{1}+\cdots+r_{5}=s} F^{\sigma}\left(j_{6}, 2-\sum_{i=1}^{5} j_{i}\right) \prod_{l=1}^{3}\left(F\left(j_{2+l}, 2-\sum_{i=1}^{l+1} j_{i}, D_{l}\right) F^{V}\left(r_{2+l}, 2-\sum_{i=1}^{1+l} r_{i}, D_{l}\right)\right) \\
& \cdot F\left(j_{2}, 2-j_{1}, B\right) F^{V}\left(r_{2}, 2-r_{1}, B\right) F\left(j_{1}, 2, A\right) F^{V}\left(r_{1}, 2, A\right) v^{0}
\end{aligned}
$$

in $Y^{*}$. For summands with $k=2$, we will implicitly assume the following. If necessary, one of the splitting operators is extrapolated to $X$, and the following operators in the concatenation (which are automatically bounded on $X$ ) are extrapolated to $Y^{*}$. In view of $(6.12)$, the formula

$$
\begin{aligned}
\left(S(\tau)-I-\tau M_{\text {ext }}\right) v^{0}= & \left(\sum_{\substack{j_{1}+\cdots+j_{6}=2 \\
r_{1}+\cdots+r_{5}=0}}+\sum_{k=0}^{2} \sum_{j_{1}+\cdots+j_{6}=k} \sum_{s=1}^{2} \sum_{r_{1}+\cdots+r_{5}=s}\right) F^{\sigma}\left(j_{6}, 2-\sum_{i=1}^{5} j_{i}\right) \\
& \cdot \prod_{l=1}^{3}\left(F\left(j_{2+l}, 2-\sum_{i=1}^{l+1} j_{i}, D_{l}\right) F^{V}\left(r_{2+l}, 2-\sum_{i=1}^{l+1} r_{i}, D_{l}\right)\right) F\left(j_{2}, 2-j_{1}, B\right) \\
& \cdot F^{V}\left(r_{2}, 2-r_{1}, B\right) F\left(j_{1}, 2, A\right) F^{V}\left(r_{1}, 2, A\right) v^{0}
\end{aligned}
$$

now follows on $Y^{*}$. The formal expression with five summation symbols means that both summation procedures are done separately, and the results are added afterwards. The remainder of the proof consists of estimates for the summands on the right hand side of (6.13) in $Y^{*}$. For convenience, the terms are sorted in the following eight groups.

(i) Let $k=2, s=0$, and let exactly one of the numbers $j_{1}, \ldots, j_{6}$ be different from zero. The summand in (6.13) for $j_{6}=2$ is $F^{\sigma}(2,2) v^{0}=\tau^{2} K_{d}^{2} \phi_{2}\left(\tau K_{d}\right) v^{0}$, and it satisfies the relations

$$
\left|\left\langle F^{\sigma}(2,2) v^{0}, y\right\rangle_{Y^{*} \times Y}\right|=\left|\left(F^{\sigma}(2,2) v^{0}, y\right)\right| \leq C \tau^{2}\left\|v_{0}\right\|_{X_{1}}\|y\|_{Y},
$$


compare (6.9). The associated expression for $j_{5}=2$ is given by $F^{\sigma}(0,0) F\left(2,2, D_{3}\right) v^{0}$. By means of Lemmas 3.7 and 3.8, as well as (6.6) and (6.9), we here derive the estimate

$$
\left|\left\langle F^{\sigma}(0,0) F\left(2,2, D_{3}\right) v^{0}, y\right\rangle_{Y^{*} \times Y}\right|=\frac{\tau^{2}}{4}\left|\left(\left(I+S_{\tau}\left(D_{3}\right)\right) D_{3} v^{0}, D_{3} \mathrm{e}^{\tau K_{d}} y\right)\right| \leq C \tau^{2}\left\|v^{0}\right\|_{X_{1}}\|y\|_{Y}
$$

All remaining cases in this index category can be treated in a similar way, additionally employing Lemma 3.11 and Corollary 3.15.

(ii) Let $k=2, s=0$, and let exactly two indices $j_{i_{1}}$ and $j_{i_{2}}$ equal one. The first representative choice is $j_{5}=j_{6}=1$ with the corresponding term $F^{\sigma}(1,1) F\left(1,2, D_{3}\right) v^{0}$. The latter satisfies the inequality

$$
\left|\left\langle F^{\sigma}(1,1) F\left(1,2, D_{3}\right) v^{0}, y\right\rangle_{Y^{*} \times Y}\right|=\tau^{2}\left|\left(K_{d} \phi_{1}\left(\tau K_{d}\right) D_{3} v^{0}, y\right)\right| \leq C \tau^{2}\left\|v^{0}\right\|_{X_{1}}\|y\|_{Y},
$$

see (6.6) and (6.9). The second instance is $j_{4}=j_{5}=1$, leading to the summand $F^{\sigma}(0,0) F\left(1,1, D_{3}\right) F\left(1,2, D_{2}\right) v^{0}$. Applying Lemma 3.8, (6.6) and (6.9), we here derive the bound

$$
\left|\left\langle F^{\sigma}(0,0) F\left(1,1, D_{3}\right) F\left(1,2, D_{2}\right) v^{0}, y\right\rangle_{Y^{*} \times Y}\right|=\frac{\tau^{2}}{2}\left|\left(\left(I+S_{\tau}\left(D_{3}\right)\right) D_{2} v^{0}, D_{3} \mathrm{e}^{\tau K_{d}} y\right)\right| \leq C \tau^{2}\left\|v^{0}\right\|_{X_{1}}\|y\|_{Y} .
$$

All other index configurations in this group are tackled in the same way, using Corollary 3.15.

(iii) Assume $k=0$ and $s=1$. It here suffices to consider only the sample summand $F^{V}\left(1,2, D_{3}\right) v^{0}$ being associated to $r_{5}=1$. In view of (6.5), Lemma 3.8, and the identity $\frac{\tau}{2} D_{3}\left(I-\frac{\tau}{2} D_{3}\right)^{-1}=-I+\left(I-\frac{\tau}{2} D_{3}\right)^{-1}$, we infer the relations

$$
\left|\left\langle F^{V}\left(1,2, D_{3}\right) v^{0}, y\right\rangle_{Y^{*} \times Y}\right|=\frac{\tau^{2}}{2}\left|\left(\frac{\tau}{2} D_{3}\left(I-\frac{\tau}{2} D_{3}\right)^{-1} v^{0},\left(I-\frac{\tau}{2} D_{3}\right)^{-1} D_{3} y\right)\right| \leq C \tau^{2}\left\|v^{0}\right\|_{X_{1}}\|y\|_{Y} .
$$

(iv) Let $k=0$ and $s=2$. In consideration of Lemma 6.1 and (6.5), it is enough to deal with the configurations $r_{4}=r_{5}=1$, respectively $r_{5}=2$. The first one leads to the summand $F^{V}\left(1,1, D_{3}\right) F^{V}\left(1,2, D_{2}\right) v^{0}$. Applying $(6.4)$, Lemma 3.8 and Corollary 3.15, the estimate

$$
\begin{aligned}
\left|\left\langle F^{V}\left(1,1, D_{3}\right) F^{V}\left(1,2, D_{2}\right) v^{0}, y\right\rangle_{Y^{*} \times Y}\right| & =\left|\left(\frac{\tau^{3}}{4} V_{\tau}^{(1)}\left(D_{3}\right) D_{2}^{2}\left(I-\frac{\tau^{2}}{4} D_{2}^{2}\right)^{-1} v^{0}, y\right)\right| \\
& =\frac{\tau^{2}}{2}\left|\left(\frac{\tau}{2} D_{2}\left(I-\frac{\tau}{2} D_{2}\right)^{-1} v^{0}, D_{2}\left(I-\frac{\tau}{2} D_{2}\right)^{-1} V_{\tau}^{(1)}\left(D_{3}\right)^{*} y\right)\right| \\
& \leq C \tau^{2}\left\|v^{0}\right\|_{X_{1}}\|y\|_{Y}
\end{aligned}
$$

is derived. The second instance $r_{5}=2$ directs to the vector $F^{V}\left(2,2, D_{3}\right) v^{0}$, which satisfies due to (6.4) the relations

$$
\left|\left\langle F^{V}\left(2,2, D_{3}\right) v^{0}, y\right\rangle_{Y^{*} \times Y}\right|=\left|\left(V_{\tau}^{(2)}\left(D_{3}\right) v^{0}, y\right)\right| \leq C \tau^{2}\left\|v^{0}\right\|_{X_{1}}\|y\|_{Y}
$$

(v) The case $k=1=s$ can be treated similar to the first configuration in (iv) by means of Lemmas 3.7, 3.8, 3.11 and 6.1, and Corollary 3.15. We in this way arrive at the same kind of estimates.

(vi) Let $k=1$ and $s=2$. In view of $(6.5),(6.6)$ and (6.9), it suffices to consider the choice $j_{5}=1$, combined with the cases $r_{5}=2$ and $r_{4}=r_{5}=1$. The first configuration results in the expression $F\left(1,2, D_{3}\right) F^{V}\left(2,2, D_{3}\right) v^{0}=$ $\tau D_{3} V_{\tau}^{(2)}\left(D_{3}\right) v^{0}$ which is of order $\tau^{3}$ in $Y^{*}$ due to (6.4). The second sample then corresponds to the vector $F\left(1,2, D_{3}\right) F^{V}\left(1,1, D_{3}\right) F^{V}\left(1,2, D_{2}\right) v^{0}$, which satisfies the relations

$$
\begin{aligned}
\left|\left\langle F\left(1,2, D_{3}\right) F^{V}\left(1,1, D_{3}\right) F^{V}\left(1,2, D_{2}\right) v^{0}, y\right\rangle_{Y^{*} \times Y}\right| & =\tau^{2}\left|\left(D_{3} V_{\tau}^{(1)}\left(D_{3}\right) \frac{\tau^{2}}{4} D_{2}^{2}\left(I-\frac{\tau^{2}}{4} D_{2}^{2}\right)^{-1} v^{0}, y\right)\right| \\
& \leq C \tau^{3}\left\|v^{0}\right\|_{X_{1}}\|y\|_{Y}
\end{aligned}
$$

employing (6.4) and Corollary 3.15.

(vii) For the case $k=2$ and $s=1$, we distinguish between the subclass of summands where exactly one index $j_{i}$ is equal to 2 , and the subclass where two indices $j_{i_{1}}$ and $j_{i_{2}}$ are equal to 1 . The two configurations $\left(j_{6}=2, r_{5}=1\right)$ and $\left(j_{5}=2, r_{5}=1\right)$ are representative for the first subclass. We consequently consider here $F^{\sigma}(2,2) F^{V}\left(1,2, D_{3}\right) v^{0}$ and $F^{\sigma}(0,0) F\left(2,2, D_{3}\right) F^{V}\left(1,2, D_{3}\right) v^{0}$, which can be estimated by means of Lemmas 3.11 and 6.1, Corollary 3.15, and the same arguments as in part (i). Concerning the second subclass, Lemma 3.11 and Corollary 3.15 imply that it is enough to deal with the two combinations $j_{5}=j_{6}=1=r_{5}$, and $j_{4}=j_{5}=1=r_{5}$, meaning the vectors $F^{\sigma}(1,1) F\left(1,2, D_{3}\right) F^{V}\left(1,2, D_{3}\right) v^{0}$ and $F^{\sigma}(0,0) F\left(1,1, D_{3}\right) F^{V}\left(1,2, D_{3}\right) F\left(1,2, D_{2}\right) v^{0}$. Employing Lemma 6.1 , the reasoning of part (ii) also here shows uniform estimates with order $\tau^{2}$ in $Y^{*}$. 
(viii) The remaining case $k=2=s$ reduces in view of Lemma 6.1 and (6.4) to the parts (i) and (ii). We thus derive for the terms in this index category the same type of estimate as before.

The case distinction (i)-(viii) and the assumption $\tau<1$ altogether imply the bound

$$
\left|\left(\left(S(\tau)-I-\tau M_{\text {ext }}\right) v^{0}, y\right)\right| \leq C \tau^{2}\left\|v^{0}\right\|_{X_{1}}\|y\|_{Y}
$$

The asserted estimate for the local error is now a consequence of (6.10), (6.11) and (6.14).

The desired convergence result for the exponentially stable ADI scheme (3.18) against the solution of the continuous system (3.1), respectively (1.1), is now a direct consequence of Lemmas 6.1 and 6.4, employing the principle of Lady Windermere's fan. This standard technique has also been employed in [8].

Theorem 6.5. Let $\varepsilon, \mu, \sigma, \eta$ satisfy (1.2) and (3.2), and let $T>0$. The global error estimate

$$
\left|\left(v^{n}-v(n \tau), y\right)\right| \leq C \tau(1+T) T \mathrm{e}^{C_{\mathrm{stab}} T}\|v(0)\|_{X_{1}}\|y\|_{Y}, \quad y \in Y,
$$

is valid for the iterates $v^{n}$ of (3.18) with initial data $v(0)=v^{0} \in X_{1}, \tau \in\left(0, \check{\tau}_{0}\right)$, and $n \in \mathbb{N}$ with $n \tau \leq T$. The numbers $C, C_{\text {stab }}, \check{\tau}_{0}>0$ depend only on $\varepsilon, \mu, \sigma, \eta$ and $Q$.

Proof. Let $\tau \in\left(0, \check{\tau}_{0}\right)$ with $\check{\tau}_{0}$ from Proposition 6.2 . The operator $S(\tau)$ again denotes the mapping associated to one step of (3.18). The error at time $n \tau$ then has the representation

$$
e_{n}:=v^{n}-v(n \tau)=S(\tau)^{n} v^{0}-\mathrm{e}^{n \tau M_{\mathrm{ext}, 1}} v^{0}=\sum_{m=0}^{n-1} S(\tau)^{m}\left(S(\tau)-\mathrm{e}^{\tau M_{\mathrm{ext}, 1}}\right) \mathrm{e}^{(n-1-m) \tau M_{\mathrm{ext}, 1}} v^{0},
$$

applying Lady Windermere's fan. This in particular means

$$
\left(e_{n}, y\right)=\sum_{m=0}^{n-1}\left(\left(S(\tau)-\mathrm{e}^{\tau M_{\mathrm{ext}, 1}}\right) \mathrm{e}^{(n-1-m) \tau M_{\mathrm{ext}, 1}} v^{0},\left(S(\tau)^{m}\right)^{*} y\right)
$$

The local error result from Lemma 6.4 now applies in view of the skewadjointness of the operators $A, B, D_{1}, D_{2}$, and $D_{3}$, Lemmas 3.11 and 3.16, Corollary 3.15, and Proposition 3.5. Consequently, the inequality

$$
\left|\left(e_{n}, y\right)\right| \leq C_{\text {loc }} \tau^{2} \sum_{m=0}^{n-1}\left\|\mathrm{e}^{(n-1-m) \tau M_{\mathrm{ext}, 1}} v^{0}\right\|_{X_{1}}\left\|\left(S(\tau)^{m}\right)^{*} y\right\|_{Y}
$$

follows with the constant $C_{\mathrm{loc}}=C_{\mathrm{loc}}(\varepsilon, \mu, \sigma, \eta, Q)$ from Lemma 6.4. The stability results from Propositions 3.5 and 6.2 finally yield the desired estimates

$$
\left|\left(e_{n}, y\right)\right| \leq C_{\text {stab }, 1} C_{\mathrm{loc}} \tau^{2} \mathrm{e}^{C_{\mathrm{stab}} T}(1+T) \sum_{m=0}^{n-1}\left\|v^{0}\right\|_{X_{1}}\|y\|_{Y} \leq C_{\mathrm{stab}, 1} C_{\mathrm{loc}} \tau T(1+T) \mathrm{e}^{C_{\mathrm{stab}} T}\left\|v^{0}\right\|_{X_{1}}\|y\|_{Y}
$$

with $C_{\text {stab,1 }}, C_{\text {stab }}$ being the stability constants from Propositions 3.5 and 6.2 .

I am grateful to my advisor Roland Schnaubelt for valuable discussions and remarks concerning this article and my ongoing research. Further, I want to thank Jonas Köhler for helpful conversations providing more insights into ADI schemes.

\section{REFERENCES}

[1] F. Abdallah, S. Nicaise, J. Valein and A. Wehbe, Uniformly exponentially or polynomially stable approximations for second order evolution equations and some applications. ESAIM: COCV 19 (3) (2013) 844-887.

[2] H. Amann, Linear and Quasilinear Parabolic Problems. Vol I. Abstract Linear Theory. Birkhäuser, Basel (1995).

[3] C. Bardos, G. Lebeau and J. Rauch, Sharp sufficient conditions for the observation, control, and stabilization of waves from the boundary. SIAM J. Control Optim. 30 (5) (1992) 1024-1065.

[4] S. Benzoni-Gavage and D. Serre, Multidimensional hyperbolic partial differential equations. First-order systems and applications. Oxford University Press, New York (2007).

[5] W. Chen, X. Li and D. Liang, Energy-conserved splitting finite-difference time-domain methods for Maxwell's equations in three dimensions. SIAM J. Numer. Anal. 48 (4) (2010) 1530-1554.

[6] M. Costabel, M. Dauge and S. Nicaise, Singularities of Maxwell interface problems. ESAIM: M2AN 33 (3) (1999), 627-649. 
[7] A. Dedner, F. Kemm, D. Kröner, C.-D. Munz, T. Schnitzer and M. Wesenberg, Hyperbolic divergence cleaning for the MHD equations. J. Comput. Phys. 175 (2) (2002) 645-673.

[8] J. Eilinghoff, T. Jahnke and R. Schnaubelt, Error analysis of an energy preserving ADI splitting scheme for the Maxwell equations. SIAM J. Numer. Anal. 57 (3) (2019) 1036-1057.

[9] J. Eilinghoff and R. Schnaubelt, Error analysis of an ADI splitting scheme for the inhomogeneous Maxwell equations. Discrete Contin. Dyn. Syst. Ser. A. 38 (11) (2018) 5685-5709.

[10] J. Eilinghoff and R. Schnaubelt, Error estimates in $L^{2}$ of an ADI splitting scheme for the inhomogeneous Maxwell equations. Preprint 32 (2017) of CRC 1173, see www.waves.kit.edu/downloads/CRC1173_Preprint_2017-32.pdf.

[11] M. Eller, Stability of the anisotropic Maxwell equations with a conductivity term. Evol. Equ. Control Theory 8(2) (2019) 343-357.

[12] K.-J. Engel and R. Nagel, One-Parameter Semigroups for Linear Evolution Equations. Springer, New York (2000).

[13] S. Ervedoza, C. Zheng and E. Zuazua, On the observability of time-discrete conservative linear systems. J. Funct. Anal. 254 (2008) 3037-3078.

[14] S. Ervedoza and E. Zuazua, Uniformly exponentially stable approximations for a class of damped systems. J. Math. Pures Appl. 91 (2009) 20-48.

[15] V. Girault and P.-A. Raviart, Finite element methods for Navier-Stokes equations. Theory and algorithms. Springer, Berlin (1986).

[16] P. Grisvard, Elliptic problems in nonsmooth domains. Pitman, Boston (1985).

[17] E. Hansen and A. Ostermann, Dimension splitting for evolution equations. Numer. Math. 108 (2008) 557-570.

[18] M. Hochbruck, T. Jahnke and R. Schnaubelt, Convergence of an ADI splitting for Maxwell's equations. Numer. Math. 129 (2015) $535-561$.

[19] M. Hochbruck and J. Köhler, On the efficiency of the Peaceman-Rachford ADI-dG method for wave-type problems, in Numerical mathematics and advanced applications, ENUMATH 2017, F.A. Radu, K. Kumar, I. Berre, J.M. Nordbotten, I.S. Pop Ed., Springer, Cham (2019) 135-144.

[20] L. Hörmander, Notions of convexity. Birkhäuser, Boston (1994).

[21] T. Kato, Perturbation theory for linear operators. Springer, Berlin (1995).

$[22]$ V. Komornik, Exact controllability and stabilization. The multiplier method. Masson; John Wiley \& Sons, Paris (1994).

[23] J. Lee and B. Fornberg, A split step approach for the 3-D Maxwell's equations. J. Comput. Appl. Math. 158 (2003) $485-505$.

[24] T. Namiki, A new FDTD algorithm based on alternating-direction implicit method. IEEE Trans. Microwave Theory Tech. 47 (10) (1999) 2003-2007.

[25] S. Nicaise, Boundary observability of a numerical approximation scheme of Maxwell's system in a cube. Collect. Math. 59 (1) (2008) $27-52$.

[26] S. Nicaise and C. Pignotti, Internal stabilization of Maxwell's equations in heterogeneous media. Abstr. Appl. Anal. 7 (2005) 791-811.

[27] K.D. Phung, Contrôle et stabilisation d'ondes électromagnétiques. ESAIM: COCV 5 (2000) 87-137.

[28] K. Ramdani, T. Takahashi and M. Tucsnak, Uniformly exponentially stable approximations for a class of second order evolution equations. ESAIM: $C O C V$ 13(3) (2007) 503-527.

[29] M. Spitz, Regularity theory for nonautonomous Maxwell equations with perfectly conducting boundary conditions. Preprint 8 (2018) of CRC 1173, see www.waves.kit.edu/downloads/CRC1173_Preprint_2018-8.pdf

[30] L. Tébou and E. Zuazua, Uniform exponential long time decay for the space semi-discretization of a locally damped wave equation via an artificial numerical viscosity. Numer. Math. 95 (2003) 563-598.

[31] M. Tucsnak and G. Weiss, Observation and control for operator semigroups, Birkhäuser, Basel (2009).

[32] X. Zhang, Exact internal controllability of Maxwell's equations. Appl. Math. Optim. 41 (2000) 155-170.

[33] F. Zheng, Z. Chen and J. Zhang, Toward the development of a three-dimensional unconditionally stable finite-difference timedomain method. IEEE Trans. Microwave Theory Tech. 48 (9) (2000) 1550-1558.

[34] E. Zuazua, Propagation, observation and control of waves approximated by finite difference methods. SIAM Review 47 (2) (2005) $197-243$.

Department of Mathematics, Karlsruhe Institute of Technology, Englerstr. 2, 76131 Karlsruhe, Germany.

E-MAIL: KONSTANTIN.ZERULLA@KIT.EDU 